\title{
聚集诱导延迟荧光材料及器件研究进展
}

\author{
黄 酬 $\dagger, a$ 邱志鹏 $\dagger, a$ 高 杨 $a$ 陈文铖 $*, a$ \\ 籍少敏 ${ }^{a}$ 霍延平 $*, a, b$ \\ ( ${ }^{a}$ 广东工业大学轻工化工学院 广州 510006) \\ ( $b$ 广东省分子聚集发光重点实验室(华南理工大学) 广州 510640)
}

\begin{abstract}
摘要 热活化延迟苂光(TADF)材料无需贵金属参与即可实现单线态和三线态激子的全利用, 成为了有机电致发光的 研究热点. 但是目前大部分 TADF 材料都表现出严重聚积诱导发光猝灭现象，这对其应用和发展不利. 聚集诱导延迟 荧光材料作为一种新型 TADF 材料, 具有独特的聚集诱导荧光增强现象引起科研工作者极大兴趣. 基于聚集诱导延迟 荧光材料分子设计及其光物理性能和器件研究具有重要意义. 将依据不同电子受体单元, 对聚集诱导延迟荧光材料的 发光原理、设计策略及其有机发光器件的最新进展进行简要综述, 并对其发展做出展望.
\end{abstract}

关键词 聚集诱导发光; 热活化延迟荧光; D-A 型分子; 有机发光二极管

\section{Research Progress on Aggregation-Induced Delayed Fluorescence in Materials and Devices}

\author{
Huang, $\mathrm{Chou}^{\dagger, a}$ \\ Qiu, Zhipeng ${ }^{\dagger, a}$ \\ Gao, Yang ${ }^{a}$ \\ Chen, Wen-Cheng ${ }^{*, a}$ \\ Ji, Shaomin ${ }^{a}$ \\ Huo, Yanping ${ }^{*, a, b}$ \\ ( ${ }^{a}$ School of Chemical Engineering and Light Industry, Guangdong University of Technology, Guangzhou 510006) \\ ( ${ }^{b}$ Guangdong Provincial Key Laboratory of Luminescence from Molecular Aggregates (South China University of \\ Technology), Guangzhou 510640)
}

\begin{abstract}
Thermally activated delayed fluorescent (TADF) materials can fully harvest both singlet and triplet excitons for light emission, becoming a research hot topic in the field of organic electroluminescence. However, the majority of fluorescent materials often suffers from aggregation-caused quenching, which is unfavorable for their application and development. As a new type of TADF material, aggregation-induced delayed fluorescence (AIDF) material exhibits aggregation-induced fluorescence enhancement, which has drawn great interest of researchers. The molecular design of AIDF materials, its photophysical properties and device research are of great significance. In this review, the recent progress of the donor-acceptor type AIDF materials based on different electron acceptors is summarized. The light-emitting mechanism, design strategy as well as the latest developments of the of AIDF materials and organic light-emitting diodes are elaborated, and their prospects in the future are also forecasted.
\end{abstract}

Keywords aggregation-induced emission; thermally activated delayed fluorescence; D-A type molecule; organic light- emitting diode

有机电致发光二极管(Organic Light-Emitting Diodes, OLEDs)由于其轻、薄、能耗低、色彩绚丽、响应
快、可柔性化等优点，在平板显示和固态照明领域表现 出巨大的应用前景 ${ }^{[1-3]}$. 自 1987 年邓青云博士 ${ }^{[4]}$ 利用真

\footnotetext{
* Corresponding author. E-mail: wencchen@gdut.edu.cn(W.-C. Chen), yphuo@gdut.edu.cn (Y. Huo)

Received January 29, 2021; revised April 6, 2021; published online April 25, 2021.

Project supported by the National Natural Science Foundation of China (Nos. U2001222, 21975055, 52003058 and 21975053 ), the Science and Technology Planning Project of Guangdong Province (No. 2019A050510042), Guangdong Basic and Applied Basic Research Foundation (Nos. 2019B1515120023, 2019B1515120035, 2021A1515010607), the Key Project of Educational Commission of the Open Fund of Guangdong Provincial Key Laboratory of Luminescence from Molecular Aggregates (No. 2019-kllma-06).

国家自然科学基金(Nos. U2001222, 21975055, 52003058, 21975053)、广东省科技计划(No. 2019A050510042)、广东省基础与应用基础研究基金(Nos. 2019B1515120023, 2019B1515120035, 2021A1515010607)、广东省分子聚集发光重点实验室开放基金(No. 2019-kllma-06)资助项目.

†共同第一作者(These authors contributed equally to this work).
} 
空热蒸镀技术发明了三明治式多层结构的电致发光 (electroluminescence, EL) 器件, OLED 技术蓬勃发展. 经 过多年的研究, 电致发光材料 OLED 领域经历了高速发 展, 从第一代传统荧光材料, 第二代贵重金属类磷光配 合物, 再到如今以热活化延迟苂光(thermally activated delayed fluorescence, TADF)材料为代表的第三代有机发 光材料. 一直以来, 更加高效、更低成本、色彩更鲜艳 的新型 OLED 材料都是人们追寻的热点.

根据自旋量子统计 ${ }^{[5]}$, 电激发产生的电子/空穴对以 $1: 3$ 的比例产生单重态和三重态激子. 在闭壳纯有机 体系, 单重态激子能迅速退激发出荧光, 三重态激子由 于自旋禁阻限制 ${ }^{[6-7]}$ 而只能以非辐射跃迁的方式回到基 态. 因此, 传统苂光材料只能利用 $25 \%$ 的单重态激子发 光, $75 \%$ 的三重态激子以非辐射的方式失活. TADF 材料 由于其最低激发单重态 $\left(\mathrm{S}_{1}\right)$ 和最低激发三重态 $\left(\mathrm{T}_{1}\right)$ 存在 较小的能级差 $\Delta E_{\mathrm{ST}}$, 如图 1 所示. 当 $\Delta E_{\mathrm{ST}}$ 足够小时, $\mathrm{T}_{1}$ 态激子吸收环境热[8-10]通过反向系间窝跃(reverse intersystem crossing, RISC), 高效地转换为 $\mathrm{S}_{1}$ 态激子, 再通 过辐射跃迁返回基态, 发出延迟苂光. 在一定条件下,
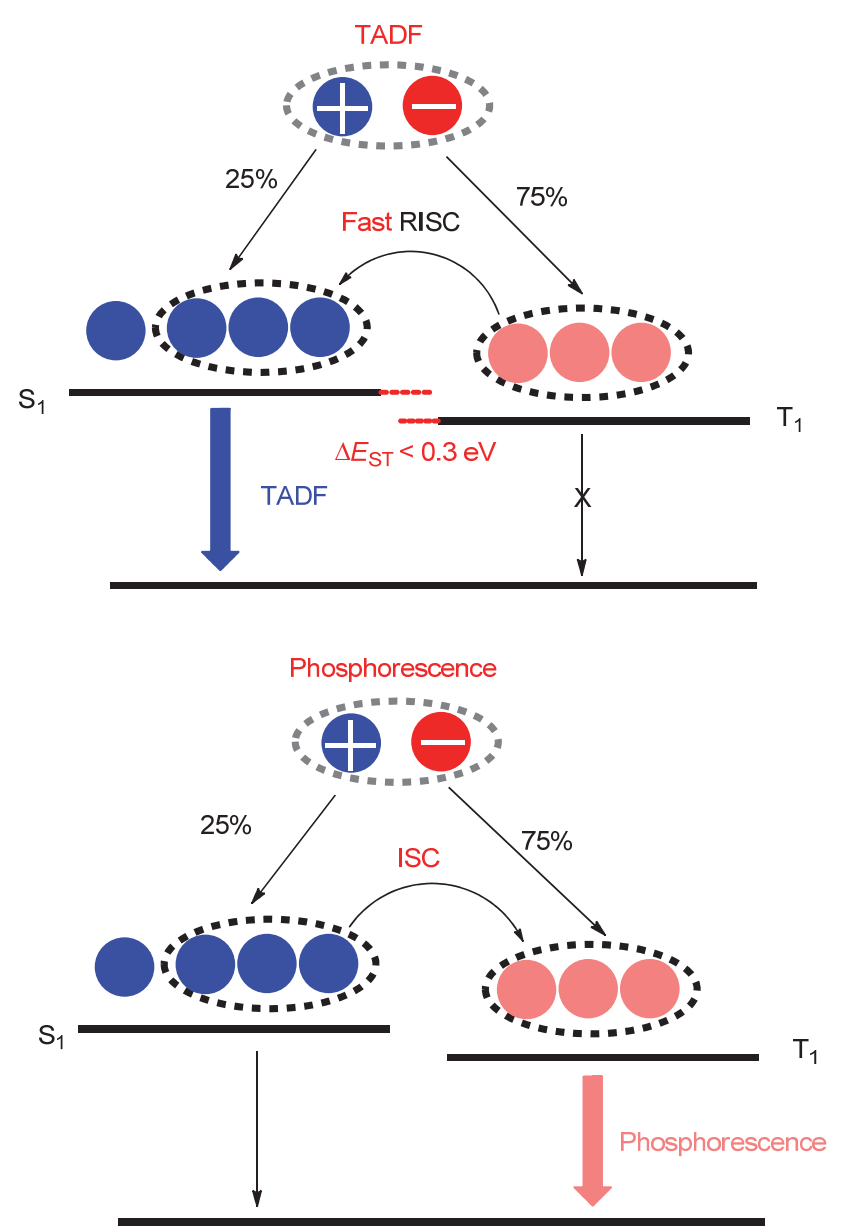

图 1 热活化延迟苂光和磷光的发光机理

Figure 1 TADF/Phosphorescence light-emitting mechanism 其内量子效率(internal quantum efficiency, IQE) 几乎达到 $100 \%$, 远超传统苂光材料 ${ }^{[1]}$. 原 $\mathrm{S}_{1}$ 态激子辐射将产生 的纳秒级别的瞬时荧光 (prompt fluorescence, PF), 而由 $\mathrm{T}_{1}$ 态经 RISC 上转换得到的激子辐射能产生微秒甚至毫 秒级别的延迟苂光 (delayed fluorescence, DF). 由于光子 发射通道一样 $\left(\mathrm{S}_{1} \rightarrow \mathrm{S}_{0}\right)$, 所以二者在发光光谱上完全一 致, 热活化延迟苂光寿命比传统荧光高两至三个数量 级.

虽然第二代磷光 OLED 采用贵金属发光配合 物 ${ }^{[2-13]}$, 在自旋耦合作用下分子内部系间窝跃(intersystem crossing, ISC)得以加强，也能获得理论上 $100 \%$ 的 $\mathrm{IQE}^{[14-17]}$, 但贵金属的添加提高了成本 ${ }^{[18-19]}$, 限制了 OLED 的进一步推广和商业化. 因此, TADF 材料已成为 有机电致发光领域的研究热点, 众多新型 TADF 分子被 相继报道, 推动着 OLED 领域的发展.

然而，TADF 材料也并非完美无缺，在该领域仍有 不少问题亟待解决. 首先, 由于 TADF 分子设计过程中 需要小的 $\Delta E_{\mathrm{ST}}$ 以进行高效的 RISC ${ }^{[11]}$, 最高占据分子轨 道(highest occupied molecular orbital, HOMO)和最低未 占据分子轨道 (lowest unoccupied molecular orbital, LUMO) 需要充分分离 ${ }^{[14,20]}$, 这将导致跃迁偶极矩减小, 辐射效率下降, 所以 TADF 分子的光致发光量子产率 (photoluminescence quantum yield, PLQY)总体并不高. 其次, 由于其本身的共轭结构, 大部分传统 TADF 分子 在聚集之后因分子之间的 $\pi-\pi$ 堆积而发生猝灭, 即产生 聚集诱导猝灭(aggregation-caused quenching, ACQ)效

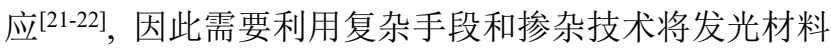
分散到主体基质中 ${ }^{[23-24]}$, 以抑制 ACQ 带来的负面影响. 而且, 由于掺杂器件在高亮度下激子浓度过高而带来的 单重态-三重态湮灭(singlet-triplet annihilation, STA)、三 重态-三重态湮灭(triplet-triplet annihilation, TTA)、三重 态一极化子湮灭(Triplet-Polaron Annihilation, TPA)效应 依然显著, 电致发光效率随亮度的提升而急剧下降 ${ }^{[25]}$. 总之, 由于激发态的长寿命, 聚集态下的浓度猝灭和激 子湮灭效应, 极大地限制了 TADF 材料的发展应用.

聚集诱导发光(Aggregation-Induced Emission, AIE) 概念的提出为解决上述问题提供可行的思路. AIE 最早 由唐本忠课题组 ${ }^{[26]}$ 在 2001 年提出, 他们在研究 1-甲基1,2,3,4,5-五苯基噻咯分子时, 发现该分子在稀溶液中发 光微弱, 而聚集态发光却显著增强, 并把这种与 ACQ 效应相反的发光现象命名为 AIE. 为研究 AIE 机理, 许 多科研工作者提出解释, 例如分子内运动受限 (restriction of intramolecular motion, RIM) ${ }^{[27]} 、 E / Z$ 异构

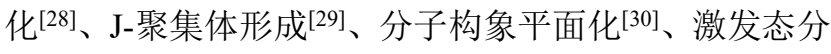

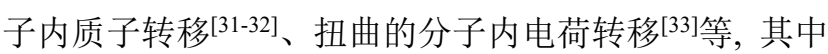


唐本忠团队提出的 RIM 机理被广大同行认可. 该机理 认为, 稀溶液状态下分子内运动活跃, 不断进行旋转和 振动, 激发态分子主要以非辐射跃迁的方式释放能量, 荧光微弱; 在分子形成聚集态后, 因空间位阻以及分子 间的相互作用, 分子运动受限, 包括分子内旋转和分子 内振动受限, 非辐射跃迁通道关闭, 激发态分子以辐射 跃迁的形式回到基态，从而发出强烈苂光.

将 AIE 机制引入 TADF 材料中, 不仅能够解决 $\mathrm{TADF}$ 器件中浓度猝灭和激子湮灭效应带来的困扰, 还 可以简化器件制作流程, 避免使用复杂掺杂技术在商业 推广中带来的困扰. 此外, 因为许多具有 AIE 性质的分 子在本质上归属于传统荧光分子，它们只能利用 $25 \%$ 的 单重态激子进行发光，根据朗伯发光模式以 $20 \% \sim 30 \%$ 的光取出效率计算, 它们的最大外量子效率 (external quantum efficiency, EQE) 也只有 $5 \% \sim 7.5 \%{ }^{[34]}$, 将 TADF 机制引入 AIE 分子中, 也能解决传统 AIE 分子电致发光 效率低下的问题.

研究发现, AIE 分子通常具有扭曲的分子结构, 聚 集态下分子间 $\pi-\pi$ 相互作用减弱，有利于抑制苂光猝灭 和激子湮灭. 同时，扭曲结构降低了分子之间的电子耦 合, 并导致弱的电子云轨道重叠. 由此可见, AIE 分子与 TADF 分子确有共通之处一一它们都具有高度扭曲的分 子构型 ${ }^{[35]}$, 某些 TADF 分子在本质上也具有 AIE 特 性 ${ }^{[14]}$. 在这种情况下, 若 AIE 分子中存在电子给体(D) 和电子受体(A)的单元部分, 其给体 $\mathrm{D}$ 上的 $\mathrm{HOMO}$ 和受 体 A 上的 LUMO 电子云能够有效分离, 从而诱导其产 生较小的 $\Delta E_{\mathrm{ST}}$ 值.

在 AIE 分子的基础上, 通过选取特定的给受体单 元, 满足单重态和三线激发态的能级接近 $(<0.30 \mathrm{eV})$ 的 前提下, 便能使这些分子展现出 AIE 和 TADF 的现象. 这种同时具有 TADF 和 AIE 性质的材料被命名为聚集诱 导延迟苂光(Aggregation-Induced Delayed Fluorescence, AIDF)材料. 具有 AIDF 的分子在稀溶液状态下发出微 弱荧光或不发射荧光, 其延迟组分可忽略不计; 而在聚 集态下(不良溶剂比例升高或者无定形薄膜)发出强烈苂 光, 伴随显著的延迟荧光寿命组分. 唐本忠课题组 ${ }^{[27]}$ 研 究指出, AIDF 现象的产生依赖内部转换(intramolecular charge, IC)的抑制以及反向系间窝跃(RISC)的促进. 具 体机理如图 2 所示, 在稀溶液状态时, 分子内的运动导 致其激发态能量主要通过 IC 等非辐射跃迁通道散失, 在此情况下 $\mathrm{IC}$ 的速率 $\left(k_{\mathrm{IC}}\right)$ 一般可达到 $10^{8}$ 到 $10^{10}$ 数量级, 大大超过其自身的荧光辐射速率 $\left(k_{\mathrm{F}}\right)$, 从而发光微弱. 此外, 由于系间䇀越过程(intersystem crossing, ISC)的速 率 $\left(k_{\mathrm{ISC}}\right)$ 同样远远小于 IC 速率, 无法形成有效的 ISC 通 道，致使 RISC 通道同样被抑制，无法产生延迟苂光. 而
当 AIDF 分子处于聚集态时，由于空间位阻增大，分子 内运动受到了限制, 致使 IC 的速率大大降低. 此时 $k_{\mathrm{F}}$ 与 $k_{\mathrm{IC}}$ 接近, 较之前能够形成有效辐射跃迁过程, 发光较 稀溶液状态下大大增强; 与此同时, ISC 与 IC 过程相互 竞争，促使 ISC、RISC 过程的产生，从而形成延迟苂光.
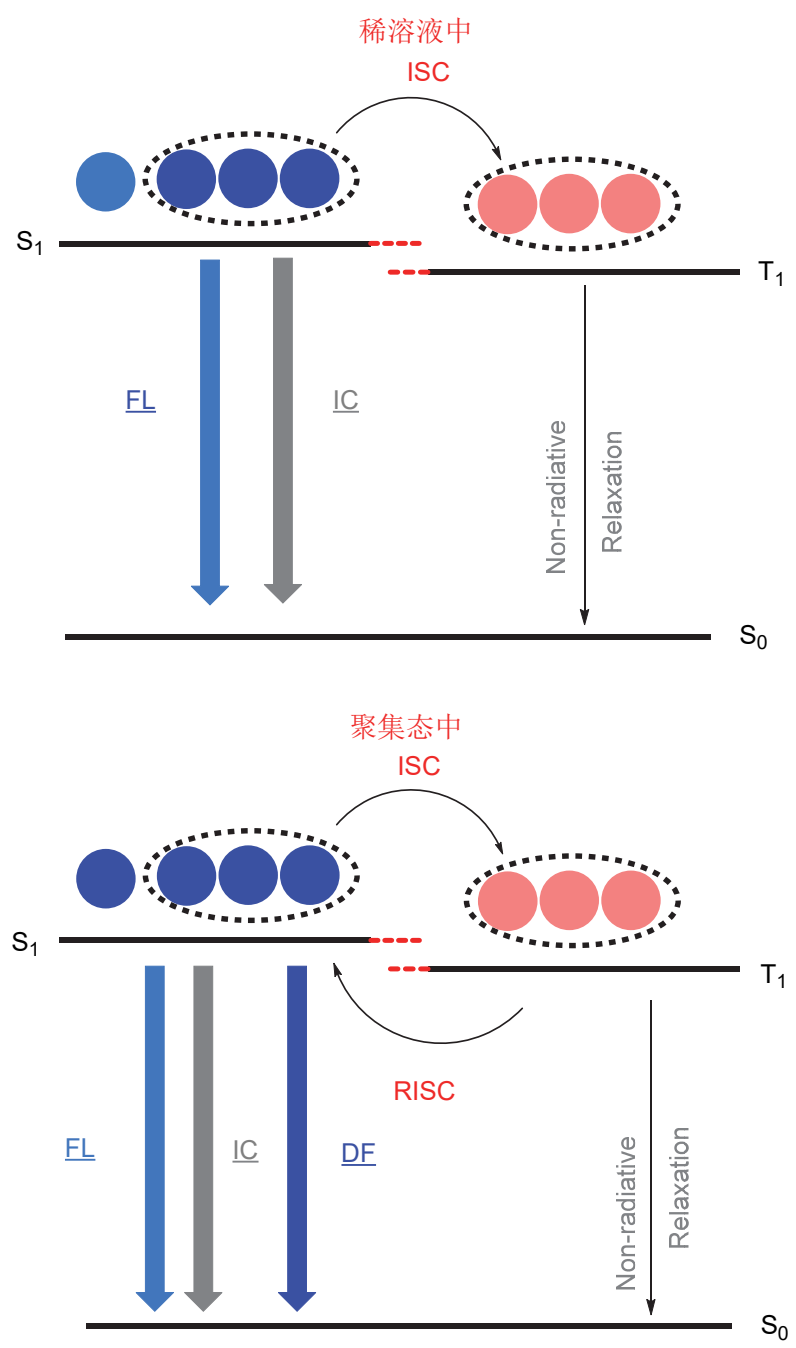

图 2 聚集诱导热活化延迟苂光机理

Figure 2 Aggregation-induced delayed fluorescence mechanism

目前常用于构筑 AIDF 分子的给体单元主要为给电 子能力较强、三线态能级较高的芳胺类基团，如咔唑、 呋啶、苯胺、吩噻嗪及其后修饰基团等. 相比之下，受 体单元可选择范围更广，不但有二苯甲酮、二苯砜、三 嗪、喹喔啉等作为受体单元构筑的衍生物, 还有基于氰 基苯、䒺啶、邻碳嗍烷等各种新型分子. 本文以受体单 元的不同为分类依据，下文将对各类分子展开介绍.

\section{1 二苯甲酮及其衍生物}

二苯甲酮(Benzophenone, BP)的中心是一个吸电子 
羰基，它与两个苯基直接相连，赋予整个基团吸电子能 力, 易于引入具有不同电子给体, 因而被广泛应用于 $\mathrm{TADF}$ 分子设计. 且因为两个苯环与羰基以单键连接, 连接供体时更易获得大的扭转角. 该类分子多采用 $\mathrm{D}-\mathrm{A}-\mathrm{D}^{\prime}$ 型设计方案, 在构建扭曲构象获得较小 $\Delta E_{\mathrm{ST}}$ 的 同时保证其拥有 AIE 性能(Scheme 1), 相关性质见表 1 .

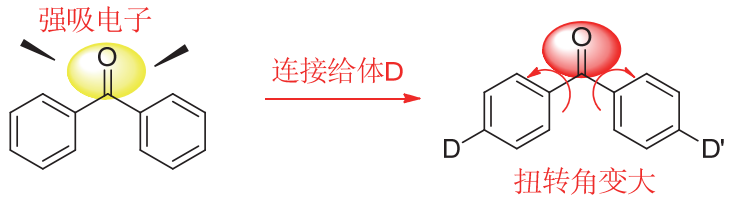

图式 1 二苯甲酮类 AIDF 分子设计策略

Scheme 1 Molecular design strategy of benzophenone-based AIDF compounds

表 1 二苯甲酮类 AIDF 分子在溶液中或分散在主体薄膜或非掺杂成膜中光致发光波长 $\left(\lambda_{\mathrm{PL}}\right)$ 、苂光量子产率 $(\mathrm{PLQY})$, 纯膜中苂光 寿命 $(\tau \mathrm{PF}, \tau \mathrm{DF})$ 、单重态-三重态能级差 $(\Delta E \mathrm{ST})$ 、非掺杂或掺杂器件电致发光波长 $(\lambda \mathrm{EL})$ 、最大外量子效率 $(\mathrm{EQE})$ 以及 $\mathrm{CIE}$ 色坐标

Table 1 PL peak $\left(\lambda_{\mathrm{PL}}\right)$ in solution or dispersed in a host film or in neat film, photoluminescence quantum yield (PLQY), lifetime in neat film $\left(\tau_{\mathrm{PF}}, \tau_{\mathrm{DF}}\right)$, singlet-triplet energy gap $\left(\Delta E_{\mathrm{ST}}\right)$ of benzophenone-AIDF, EL peak $\left(\lambda_{\mathrm{EL}}\right)$ and maximum EQE, CIE for nondoped or doped devices, respecyively

\begin{tabular}{|c|c|c|c|c|c|c|c|c|c|}
\hline Compd. & $\lambda \mathrm{PL} / \mathrm{nm}$ & PLQY $/ \%$ & $\tau \mathrm{PF} / \mathrm{ns}$ & $\tau \mathrm{DF} / \mu \mathrm{s}$ & $\Delta E_{\mathrm{ST}} / \mathrm{eV}$ & $\lambda \mathrm{EL} / \mathrm{nm}$ & $\mathrm{EQE} / \%$ & CIE & Ref. \\
\hline 1 & $509 / 505$ & $8.3 / 80.2$ & 40.4 & 2.9 & 0.08 & $516 / 508$ & $14.2 / 17.9$ & $(0.26,0.55) /(0.23,0.51)$ & {$[36]$} \\
\hline 2 & $582 / 530$ & $2.2 / 58.0$ & 23.5 & 2.1 & 0.024 & $548 / 528$ & $18.4 / 22.3$ & $(0.40,0.57) /(0.32,0.57)$ & {$[37]$} \\
\hline 3 & $567 / 538$ & $1.2 / 45.3$ & 19.1 & 5.7 & 0.033 & $554 / 528$ & $15.3 / 22.7$ & $(0.42,0.55) /(0.33,0.58)$ & {$[37]$} \\
\hline 4 & $518 / 490$ & $18.2 / 67.4$ & 21.9 & 5.5 & 0.016 & $502 / 542$ & $15 / 19.1$ & $(0.23,0.49) /(0.37,0.57)$ & {$[37]$} \\
\hline 5 & $550 / 524$ & $0.8 / 22.0$ & 53.5 & 0.94 & 0.08 & $549 /-$ & $5.9 / 15.9$ & $(0.41,0.54) /(0.37,0.53)$ & [38] \\
\hline 6 & $543 / 520$ & $1.9 / 24.2$ & 40.8 & 0.91 & 0.09 & $541 /-$ & $6 / 15.4$ & $(0.38,0.55) /(0.34,0.53)$ & {$[38]$} \\
\hline 7 & $535 / 518$ & - & 34.2 & 8.1 & 0.1 & $522 / 522$ & $8.15 / 19.67$ & $(0.28,0.59) /(0.26,0.56)$ & [39] \\
\hline 8 & $595 / 548$ & $2.6 / 45.4$ & 30.2 & 1.4 & - & $560 /-$ & $13.3 / 18.6$ & $(0.44,0.54) /(0.40,0.57)$ & {$[27]$} \\
\hline 9 & $596 / 550$ & $2.8 / 48.7$ & 27.9 & 1.1 & - & $560 /-$ & $14.3 / 19$ & $(0.46,0.53) /(0.40,0.56)$ & {$[27]$} \\
\hline 10 & $596 / 551$ & $1.8 / 45.5$ & 27.5 & 1.1 & - & $560 /-$ & $12.3 / 19.4$ & $(0.46,0.53) /(0.42,0.56)$ & {$[27]$} \\
\hline 11 & $474 / 480$ & $-/ 38.6$ & 6.8 & 1.6 & 0.18 & $494 / 489$ & $4.4 / 7.1$ & $(0.21,0.42) /(0.18,0.39)$ & {$[40]$} \\
\hline 12 & $533 / 527$ & $-/ 60.5$ & 17.8 & 2.4 & 0.17 & $555 / 540$ & $8.2 / 13.6$ & $(0.41,0.55) /(0.36,0.59)$ & {$[40]$} \\
\hline 13 & $508 / 498$ & $-/ 78.0$ & 13 & 1870 & 0.4 & $534 / 520$ & $3.8 / 18.8$ & $(0.35,0.56) /(0.30,0.53)$ & {$[41]$} \\
\hline 14 & $563 / 535$ & $-/ 65.0$ & 18.4 & 2.3 & 0.07 & $580 / 550$ & $7.5 / 16.6$ & $(0.49,0.49) /-$ & [41] \\
\hline 15 & -1518 & $23.5 / 72.1$ & 22.1 & 4.9 & 0.06 & $528 / 560$ & $20.1 / 26.8$ & $(0.32,0.58) /(0.48,0.52)$ & {$[42]$} \\
\hline 16 & $576 / 530$ & $3.9 / 69.0$ & 22.4 & 2.6 & 0.024 & $548 /-$ & $22.6 /-$ & $(0.39,0.57) /-$ & [45] \\
\hline 17 & $575 / 532$ & $3.0 / 71.6$ & 21.1 & 2.4 & 0.02 & $546 /-$ & $21.4 /-$ & $(0.39,0.57) /-$ & [45] \\
\hline 18 & $575 / 529$ & $3.1 / 66.0$ & 23.7 & 2.3 & 0.024 & $542 /-$ & $22.1 /-$ & $(0.39,0.57) /-$ & [45] \\
\hline 19 & $574 / 530$ & $2.8 / 71.2$ & 23 & 2.4 & 0.016 & $542 /-$ & $21.8 /-$ & $(0.38,0.57) /-$ & {$[45]$} \\
\hline 20 & $568 / 530$ & $2.2 / 66.5$ & 412.8 & 1.58 & 0.021 & $538,544 /-$ & $\begin{array}{c}17.3 、 16.3 / \\
-\end{array}$ & $\begin{array}{l}(0.39,0.57) 、 \\
(0.37,0.57) /-\end{array}$ & {$[46]$} \\
\hline 21 & $569 / 533$ & $2.7 / 67.9$ & 388.9 & 1.68 & 0.013 & $542,540 /-$ & $16.1 、 16.5 /-$ & $\begin{array}{l}(0.38,0.57) \\
(0.37,0.58) /-\end{array}$ & {$[46]$} \\
\hline 22 & $404 、 430 /-$ & $-/ 30.4$ & - & 2.4 & 0.262 & $460,600 /-$ & $0.7 /-$ & - & {$[47]$} \\
\hline 23 & $476 /-$ & $-/ 45.2$ & - & 4 & 0.264 & $460,600 /-$ & $1.6 /-$ & - & {$[47]$} \\
\hline 24 & $624 / 574$ & $3.3 / 30.2$ & 21.4 & 0.8 & 0.045 & $570 / 545$ & $7.04 / 15.77$ & $(0.42,0.55) /(0.31,0.57)$ & {$[48]$} \\
\hline 25 & $637 / 586$ & $1.2 / 20.5$ & 22.3 & 0.7 & 0.078 & $576 / 548$ & $6.9 / 11.7$ & $(0.51,0.48) /(0.42,0.57)$ & {$[48]$} \\
\hline 26 & $560 / 520$ & $8.8 / 15.2$ & 24.8 & 1.7 & 0.27 & $528 / 513$ & $2.58 / 5.05$ & $(0.39,0.54) /(0.28,0.57)$ & [48] \\
\hline 27 & $600 / 580$ & $0.51 / 31.48$ & 10.4 & 0.79 & 0.03 & $592 / 550$ & $4.94 / 13.31$ & $(0.54,0.45) /(0.45,0.53)$ & [49] \\
\hline 28 & $610 / 589$ & $1.14 / 32.32$ & 10.2 & 0.52 & 0.02 & $606 / 562$ & $3.71 / 13.75$ & $(0.57,0.42) /(0.44,0.53)$ & [49] \\
\hline 29 & $582 / 547$ & $4.1 / 38.3$ & 22.9 & 1.2 & 0.02 & $505 / 499$ & $9.02 / 10.03$ & $(0.27,0.50) /(0.24,0.48)$ & {$[50]$} \\
\hline 30 & $525 / 510$ & $11.7 / 59.5$ & 25.7 & 2.9 & 0.04 & $568 / 524$ & $7.73 / 12.13$ & $(0.45,0.52) /(0.34,0.56)$ & {$[50]$} \\
\hline 31 & $501 / 510$ & $14.0 / 51.0$ & 14.2 & 0.79 & 0.06 & $530 /-$ & $6.4 /-$ & - & {$[51]$} \\
\hline 32 & $498 / 512$ & $15.2 / 44.0$ & 14.9 & 0.54 & 0.09 & $530 /-$ & $9.8 /-$ & - & [51] \\
\hline 33 & $-/ 480$ & $2.3 / 39.8$ & 25.4 & 1.7 & 0.1 & $482 /-$ & $2.9 /-$ & $(0.19,0.30) /-$ & {$[52]$} \\
\hline 34 & $-/ 490$ & $1.6 / 55.7$ & 23.7 & 1.5 & 0.07 & $494 /-$ & $3.7 /-$ & $(0.24,0.41) /-$ & {$[52]$} \\
\hline 35 & -1528 & $0.8 / 58.6$ & 24.3 & 1.6 & 0.05 & $529 /-$ & $4.3 /-$ & $(0.36,0.56) /-$ & {$[52]$} \\
\hline 36 & $-/ 475$ & $2.4 / 46.6$ & 13.3 & 1.1 & 0.07 & $486 /-$ & $4.8 /-$ & $(0.22,0.31) /-$ & {$[52]$} \\
\hline 37 & $-/ 487$ & $1.9 / 64.8$ & 11.7 & 0.9 & 0.06 & $502 /-$ & $8.2 /-$ & $(0.27,0.46) /-$ & {$[52]$} \\
\hline 38 & $-/ 525$ & $1.1 / 66.5$ & 12.5 & 0.8 & 0.04 & $535 /-$ & $12.1 /-$ & $(0.38,0.56) /-$ & {$[52]$} \\
\hline
\end{tabular}


2017 年, 赵祖金课题组 ${ }^{[36]}$ 通过将二苯并噻吩 (dibenzothiophene, DBT)和 9,9-二甲基-9,10-二氢唤啶 (9,9-dimethyl-9,10-dihydroacridine, DMAC)连接到苯甲 酰基(benzoyl, BZ)上构建了不对称 D-A-D'分子 1. 不对 称的 D-A-D'结构由于其双给体的存在, 可通过引入不 同的给体, 从而调控材料的光学性质、能级、载流子输 运以及分子间距离和相互作用. 采用平面二苯并噻吩 (DBT) 作为固定给体, 提高了材料的电荷输运能力. DMAC 也具有良好的给电性和空穴输运能力, 能够促 进 HOMO 和 LUMO 与 $\mathrm{BZ}$ 受体的分离. 得益于合理的 分子设计, HOMO 和 LUMO 的重叠程度被有效控制, 该 分子在纯膜中的 $\Delta E_{\mathrm{ST}}$ 仅为 $0.08 \mathrm{eV}$. 它在四氢呋喃(THF) 溶液中发光微弱, PLQY 仅有 $8.3 \%$, 但在纯膜中却能发 出强绿光, PLQY 高达 $80.2 \%$, 较掺杂薄膜更高, 且纯膜 中表现出明显的延迟寿命 $(2.9 \mu \mathrm{s})$, 表现出 TADF 性能. 结构为 ITO/TAPC (25 nm)/EML (35 nm)/TmPyPB (55 $\mathrm{nm}) / \mathrm{LiF}(1 \mathrm{~nm}) / \mathrm{Al}$ 的非掺杂器件, 最大电流效率 (Maximum current efficiency, CE) 和最大功率(Maximum power efficiency, $\mathrm{PE}$ )效率分别为 $43.3 \mathrm{~cd} \cdot \mathrm{A}^{-1}$ 和 35.7 $1 \mathrm{~m} \bullet \mathrm{W}^{-1}, \mathrm{EQE}$ 为 $14.2 \%$, 并在 $1000 \mathrm{~cd} \bullet \mathrm{m}^{-2}$ 下表现出极低 的效率滚降, 仅为 $0.46 \%$. 这是当时效率滚降最低的非 掺杂的 TADF-OLED 之一, 证明将 AIE 和 TADF 优点的 结合是一种可行的分子设计策略, 用于探索高效稳定、 无需掺杂的 OLED 理想发光材料.

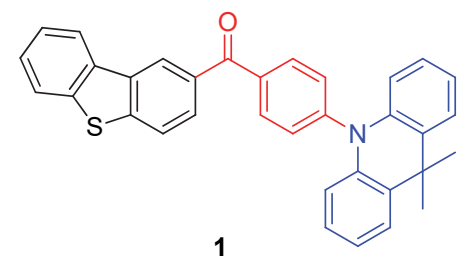

同年, 为进一步探索 AIE-TADF 结合的可行性及优 势, 赵祖金课题组 ${ }^{[37]}$ 利用 $\mathrm{BP}$ 作受体, 在分子 $\mathbf{1}$ 的基础 上, 将固定给体 $\mathrm{DBT}$ 换为给电子能力更加匹配和平面 性更好的 9-苯基-9 氢咔唑 (9-Phenyl-9H-carbazole, $\mathrm{Ph}-\mathrm{Cz}$ ), 选取吩噻嗪(phenoxazine, PXZ)、吩噁嗪(phenothiazine, PTZ)和 DMAC 分别作供体，设计了分子 2、3、 4. 由于 $\mathrm{Ph}-\mathrm{Cz}$ 和苯酰基之间更大的二面角, 分子 $2 、 3$ 的 HOMO 和 LUMO 分离程度较分子 $\mathbf{1}$ 的大, 它们的 $\Delta E_{\mathrm{ST}}$ 低至 $0.024 、 0.033 、 0.016 \mathrm{eV}$. 上述分子在 $\mathrm{THF}$ 溶 液中的 PLQY 分别为 $2.2 \% 、 1.2 \% 、 18.2 \%$, 在纯膜中则 分别提升至 $58.0 \%, 45.3 \%, 67.4 \%$, 具有明显的延迟寿命 (分别为 $2.1 、 5.7 、 5.5 \mu \mathrm{s}$ ), 表现出典型的 AIDF 现象. 以 2 为发射层的非掺杂器件表现出优良的 EL 性能, 最大 外部量子效率、电流效率和功率效率分别为 $18.4 \%, 59.1$ $\mathrm{cd} \cdot \mathrm{A}^{-1}, 65.7 \mathrm{~lm} \cdot \mathrm{W}^{-1}$, 在 $1000 \mathrm{~cd} \cdot \mathrm{m}^{-2}$ 的效率滚降仅为
$1.2 \%$, 这是当时器件效率滚降最低的非掺杂 OLED. 基 于上述分子非掺杂器件性能的优异表现，作者在此明确 提出 AIDF 的概念.

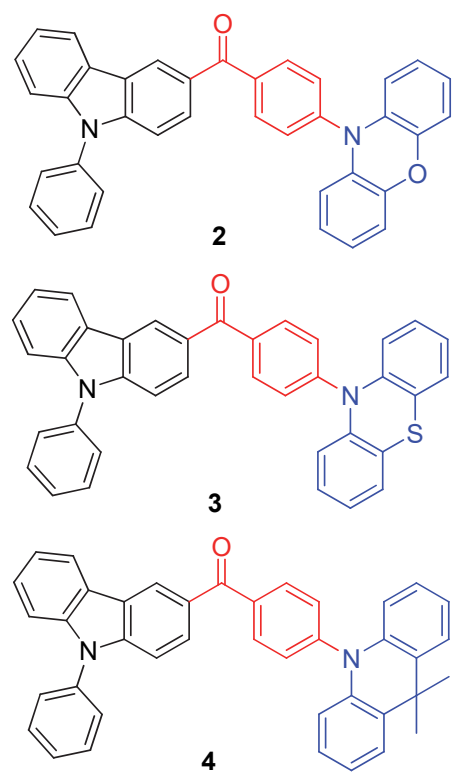

2018 年, 王利祥等 ${ }^{[38]}$ 利用 Triazatruxene (TAT)构建 了 5 和 6, TAT 是由 3 个咔唑单元组成的大刚性芳香苯环, 具有对称平面 $\pi$ 延伸共轭结构, 是一个强给体单元. 两 个分子同时具有 TADF、AIE、力致发光变色(Mechanochromic luminescence, MCL)的特性. 相似的扭曲构型以 及能级分布使两个分子表现出类似的 TADF 现象, 两个 分子的 $\Delta E_{\mathrm{ST}}$ 分别为 $0.08 、 0.09 \mathrm{eV}$, 在纯膜中的苂光量子 产率为 $22.0 \% 、 24.2 \%$, 延迟寿命为 $0.94 、 0.91 \mu \mathrm{s}$. 由于 己基和苯基的差别, 两者表现出不同的 AIE 和 MCL 现 象. 分子 5 以随机的方式聚集成团，形成具有小的分子 间阻力和较高分子重组能的无定形松散状态，导致了发 射红移，而苯基取代的分子 $\mathbf{6}$ 则由于庞大的苯基取代增 加了空间位阻，减少了分子间相互作用，降低了堆积效 率，更倾向于随机聚集，仅导致发光亮度增强，没有改 变发射波长. 以 ITO/PEDOT:PSS $(25 \mathrm{~nm}) / \mathbf{5}$ 或 6 (25 $\mathrm{nm}) / \mathrm{TmPyPB}(55 \mathrm{~nm}) / \mathrm{LiF}(1 \mathrm{~nm}) / \mathrm{Al}(150 \mathrm{~nm})$ 为结构制 备了非掺杂 OLED, 其中以 6 为发射层的器件表现出更 好的 $\mathrm{EL}$ 性能, CE、PE 和 $\mathrm{EQE}$ 分别为 $18.9 \mathrm{~cd} \bullet \mathrm{A}^{-1}, 19.21$ $\mathrm{lm} \cdot \mathrm{W}^{-1}$ 和 $6.0 \%$, 在亮度为 $1000 \mathrm{~cd} \cdot \mathrm{m}^{-2}$ 下也有 $5.8 \%$ 的 $\mathrm{EQE}$, 效率滚降为 $3.3 \%$.

随后, 施和平课题组 ${ }^{[39]}$ 以咔唑 $(\mathrm{Cz})$ 为骨架, $\mathrm{BP}$ 作受 体, DMAC 作供体设计了 $\mathrm{AIDF}$ 分子 7, 由于 $\mathrm{DMAC}$ 的 给电子能力较 PXZ 弱, HOMO 和 LUMO 电子云分布仍 存在部分重叠, 延迟寿命较上述分子长 $(8.1 \mu \mathrm{s})$. 以 $\mathrm{ITO} /$ HATCN (20 nm)/TAPC (30 nm)/EML (25 nm)/TmPyPB $(40 \mathrm{~nm}) / \mathrm{LiF}(1 \mathrm{~nm}) / \mathrm{Al}$ 为结构的非掺杂器件有着 26.88 


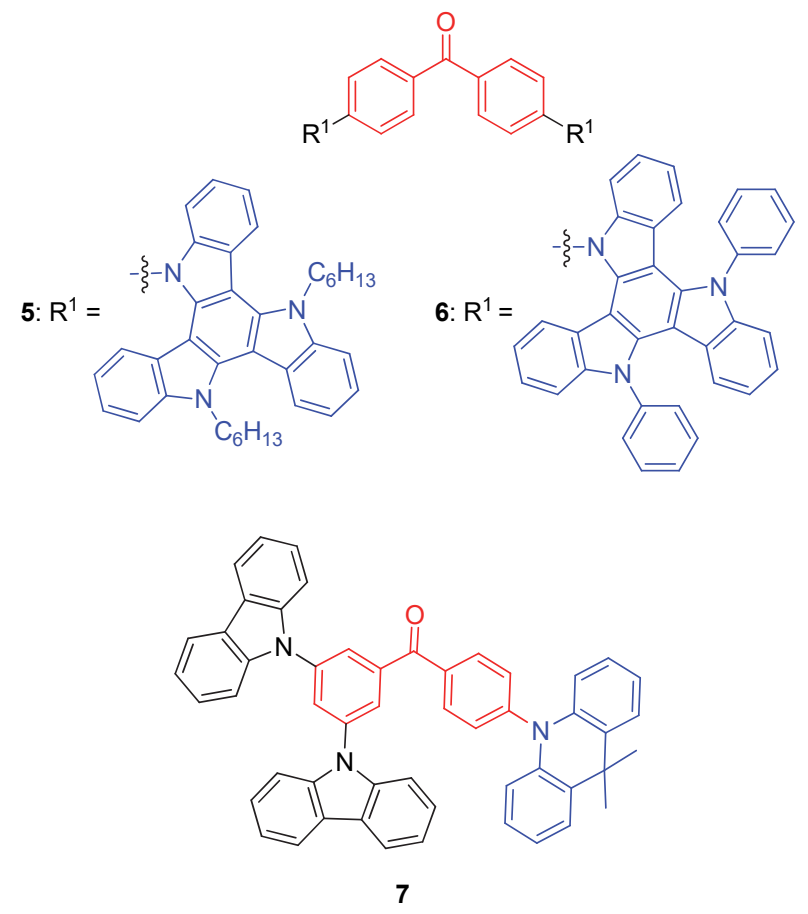

$\mathrm{cd} \cdot \mathrm{A}^{-1}, 15.63 \mathrm{~lm} \cdot \mathrm{W}^{-1}$ 以及 $8.15 \%$ 的 $\mathrm{EQE}$. 掺杂器件 ITO/HATCN $(20 \mathrm{~nm}) /$ TAPC $(30 \mathrm{~nm}) / \mathrm{EML}(w=20 \%)$ : CBP (25 nm)/TmPyPB (40 nm)/LiF (1 nm)/Al 同样显示出 良好 $\mathrm{EL}$ 性能, $\mathrm{CE} 、 \mathrm{PE}$ 和 $\mathrm{EQE}$ 分别为 $61.83 \mathrm{~cd} \cdot \mathrm{A}^{-1}, 40.45$ $\mathrm{lm} \cdot \mathrm{W}^{-1}$ 和 $19.67 \%$.

AIDF 材料在非掺杂 OLED 应用领域已展现出独特 的优势, 但其机制还有待深入研究. 2019 年, 赵祖金课 题组 ${ }^{[27]}$ 通过 Bz、PTZ 以及荡的衍生物设计了 $8 \sim 10$, 以 解释 AIDF 产生的机制. 通过溶液和纯膜中光物理参数 的比较, 作者指出, AIDF 的产生归因于聚集态下 IC 的 抑制和 ISC 的促进, 其实质则是聚集态下分子内运动受 限和分子结构的刚性所致. 且作者还发现分子 $\mathbf{8}$ 表现出 反 “卡莎” (Kasha's rule)规则行为. 卡莎规则指出, 多重 态分子的激发态电子仅能由最低激发态发射, 而分子 $\mathbf{8}$ 的有效发射并不源于 $S_{1}$, 而是来自于更高的能级, 例如 $\mathrm{S}_{2}$. 三个分子的固态 PLQY 分别为 $45.4 \% 、 48.7 \% 、 45.5 \%$, 延迟寿命为 $1.4 、 1.1 、 1.1 \mu \mathrm{s}$. 非掺杂器件结构为 ITO/ TAPC $(25 \mathrm{~nm}) / \mathrm{EML}(35 \mathrm{~nm}) / \mathrm{TmPyPB}(55 \mathrm{~nm}) / \mathrm{LiF}(1$ $\mathrm{nm}) / \mathrm{Al}$, 三种器件都具有良好的 EL 性能, 尤以 9 为最 佳, 最大 $\mathrm{EQE}$ 为 $14.3 \%$.

祁争健课题组 ${ }^{[40]}$ 以三苯胺为给体, BP 衍生物为受 体, 设计了 11 和 12 , 它们同时具有分子内化学键电荷 转移(through-bond charge transfer, TBCT)与空间电荷转 移(through-space charge transfer, TSCT)双属性. 与 $\mathrm{TBCT}$ 不同的是, TSCT 通过供体和受体单元之间的 $\pi-\pi$ 空间相互作用发生电荷转移. 一方面, TSCT 增强了 $\mathrm{CT}$ 过程, 使 HOMO 和 LUMO 分离从而减小 $\Delta E_{\mathrm{ST}}$; 另一<smiles>CC1(C)c2ccccc2-c2ccc(C(=O)c3ccc(N4c5ccccc5Oc5ccccc54)cc3)cc21</smiles><smiles>O=C(c1ccc(N2c3ccccc3Oc3ccccc32)cc1)c1ccc2c(c1)C(c1ccccc1)(c1ccccc1)c1ccccc1-2</smiles><smiles>O=C(c1ccc(N2c3ccccc3Oc3ccccc32)cc1)c1ccc2c(c1)C1(c3ccccc3-2)c2ccccc21</smiles><smiles>O=C(c1ccc2sc3ccccc3c2c1)c1cc(-c2ccc(N(c3ccccc3)c3ccccc3)cc2)ccc1-c1ccc(N(c2ccccc2)c2ccccc2)cc1</smiles><smiles>O=C(c1ccc2sc3ccccc3c2c1)c1cc(-c2ccc(N(c3ccccc3)c3ccccc3)cc2)c(C(=O)c2ccc3sc4ccccc4c3c2)cc1-c1ccc(N(c2ccccc2)c2ccccc2)cc1</smiles>

方面, TBCT 促使 HOMO 和 LUMO 在桥联基团重叠, 得 益于给受体间 $p-p$ 轨道的重叠, 使 LUMO 电子云部分迁 移至给体，从而促进辐射跃迁的产生，有利于获得高的 激子利用率. 两个分子的 $\Delta E_{\mathrm{ST}}$ 分别为 0.18 和 $0.17 \mathrm{eV} .12$ 由于拥有两个受体单元，不仅增强了分子内 D-A 的相互 作用, 也因为较高程度的构型扭曲而减弱了分子间的相 互作用，从而获得更高的 PLQY (60.5\%). 相比之下, 11 的 PLQY 为 $38.6 \%$. 非掺杂器件结构为 ITO/HATCN (5 $\mathrm{nm}) / \mathrm{TAPC}(30 \mathrm{~nm}) / \mathrm{EML}(35 \mathrm{~nm}) / \mathrm{TmPyPB}(40 \mathrm{~nm}) / \mathrm{LiF}$ (1 
$\mathrm{nm}) / \mathrm{Al}(100 \mathrm{~nm})$, 基于 $\mathbf{1 2}$ 的器件获得最好的 EL 性能, 最大 $\mathrm{CE} 、 \mathrm{PE}$ 和 $\mathrm{EQE}$ 分别为 $25.6 \mathrm{~cd} \cdot \mathrm{A}^{-1}, 15.2 \mathrm{~lm} \cdot \mathrm{W}^{-1}$ 以及 $8.2 \%$. 这种基于 AIDF 的策略为开发新型高效的 TADF 材料制造非掺杂 OLED 提供了可能.

杨楚罗课题组 ${ }^{[41]}$ 利用一个新的受体 5,6-二氢吡咯并 [2,1- $a]$ 异喹啉-1,3-二苯甲酮设计了 13 和 14.14 具有小的 $\Delta E_{\mathrm{ST}}(0.06 \mathrm{eV})$, 确保了高效的 TADF 过程, 延迟寿命 2.3 $\mu \mathrm{s}$. 相比之下, 13 的 $\Delta E_{\mathrm{ST}}$ 较大 $(0.33 \mathrm{eV})$, 延迟寿命高达 $1.87 \mathrm{~ms}$. 此外, 13 因为 DMAC 给体部分的非紧密堆积模 式以及 $\pi-\pi$ 相互作用, 使其容易在外加力作用下无序排 列, 是表现出多色 MCL 现象的内在原因, 而 PTZ 较为 刚性的结构使其分子间相互作用较少, 从而形态转变较 难发生. 掺杂器件结构为 ITO/TAPC (30 nm)/TCTA (5 $\mathrm{nm}) / \mathrm{CBP}: \mathrm{EML}(w=9 \%, 15 \mathrm{~nm}) / \mathrm{TmPyPB}(65 \mathrm{~nm}) / \mathrm{LiF}(1$ $\mathrm{nm}) / \mathrm{Al}(100 \mathrm{~nm})$, 基于 $\mathbf{1 3}$ 的器件显示出更好的 EL 性能, 最大 $\mathrm{EQE}$ 为 $18.8 \%$. 非掺杂 OLED 中, 得益于较小的 $\Delta E_{\mathrm{ST}}$, 基于 14 的器件获得了 $7.5 \%$ 的最大 $\mathrm{EQE}$.

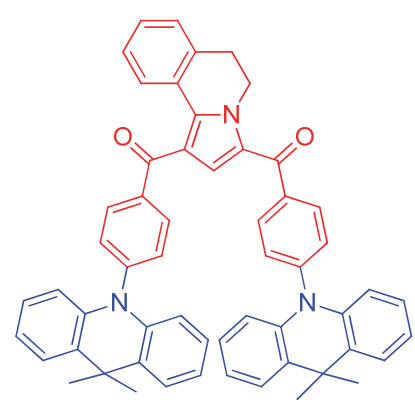

13

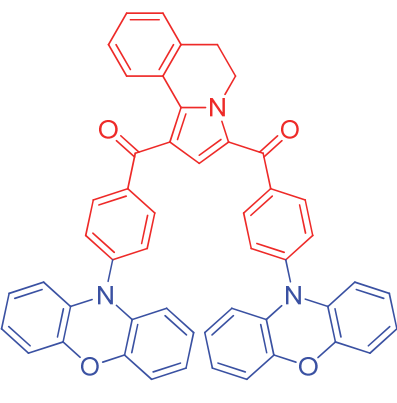

14
赵祖金课题组 ${ }^{[42]}$ 利用 DMAC 作供体, Bz 作受体设 计了分子 15. 其 $\Delta E_{\mathrm{ST}}$ 为 $0.059 \mathrm{eV}$, 在纯膜中的 PLQY 为 $72.1 \%$, 延迟寿命为 $4.9 \mu \mathrm{s}$. 由于引入了大体积的螺二芴 结构，聚集后分子间相互作用被抑制，进而减弱 TTA、 TPA 等影响, 有利于获得更高的 EQE. 非掺杂器件结构 为 ITO/HATCN $(5 \mathrm{~nm}) / \mathrm{NPB}(30 \mathrm{~nm}) / \mathrm{mCP}(10 \mathrm{~nm}) / \mathrm{SBF}-$ BP-DMAC $(30 \mathrm{~nm}) / \mathrm{TPBi}(50 \mathrm{~nm}) / \mathrm{LiF}(1 \mathrm{~nm}) / \mathrm{Al}(120$ $\mathrm{nm})$, 最大 CE、 $\mathrm{PE} 、 \mathrm{EQE}$ 分别为 $67.2 \mathrm{~cd} \cdot \mathrm{A}^{-1}, 65.9 \mathrm{~lm} \bullet$ $\mathrm{W}^{-1}$ 以及 $20.1 \%$. 基于该分子的掺杂器件获得 $26.8 \%$ 的 EQE. 同时, 由于该分子具有双极传输能力, 将其作为 主体材料制备的器件收获了 $21.0 \%$ 的 EQE.

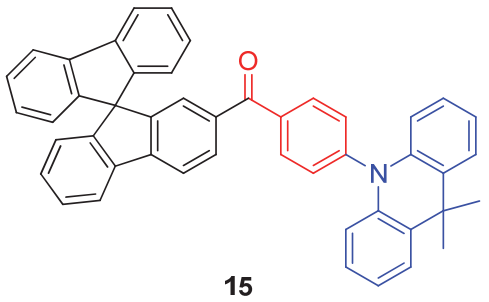

由于高电流密度引起高激子浓度，掺杂技术常常伴 随着双分子猝灭过程，导致掺杂 OLED 的效率急剧下 降 ${ }^{[43-44]}$. 赵祖金课题组 ${ }^{[45]}$ 提出一种新型的 AIDF 分子设 计，将主体材料片段接枝到 AIDF 体系中，以抑制苂光 分子的浓度猝灭，为上述问题提供了可行的解决思路. 为了验证该猜想，他们以吩啞嗪苯酰基为固定单元，分 别与不同主体材料如 CBP (4,4'-bis(carbazol-9-yl)biphenyl)和 mCBP (1,3-bis(carbazol-9-yl)benzene)等分子结 合，合成了一系列新型苂光分子 16 19. 根据理论计 算，四个分子前线轨道分布充分分离，即 HOMO 主要集 中在 PXZ 上, 而 LUMO 则位于苯甲酰和部分咔唑上. 外周咔唑和苯基对 HOMO 和 LUMO 几乎没有贡献. 在 THF 溶液中它们表现出弱的荧光(PLQY 2.8\% 3.9\%), 延迟菼光可忽略不计; 但在纯膜中显示强苂光，高的 PLQY $(66 \% \sim 71.6 \%)$ 以及足够小的 $\Delta E_{\mathrm{ST}} \quad(0.016 \sim 0.024$ $\mathrm{eV})$, 伴随着微秒级的延迟苂光寿命 $(2.3 \sim 2.6 \mu \mathrm{s})$, 证实 了 AIDF 性质. 以四种材料为发光层(EML)制备的非掺 杂 OLED, 结构为 ITO/TAPC $(25 \mathrm{~nm}) / \mathrm{EML}(35 \mathrm{~nm}) /$ TmPyPB $(55 \mathrm{~nm}) / \mathrm{LiF}(1 \mathrm{~nm}) / \mathrm{Al}$, 都表现出了出色的 $\mathrm{EL}$ 效率，成为了当时最高效的非掺杂 OLED. 以 16 为例, 它的最大亮度达 $95577 \mathrm{~cd} \cdot \mathrm{m}^{-2}, \mathrm{EQE} 、 \mathrm{CE} 、 \mathrm{PE}$ 分别为 $22.6 \%, 72.9 \mathrm{~cd} \cdot \mathrm{A}^{-1}, 81.8 \mathrm{~lm} \cdot \mathrm{W}^{-1}$, 在 $5000 \mathrm{~cd} \cdot \mathrm{m}^{-2}$ 的亮度 下效率滚降仅为 $11.4 \%$. 四种器件还收获了将近 $100 \%$ 的激子，进一步的研究分析表明，接枝主体材料基团后， 载流子的注入、传输和复合能力大大提升, 证实该策略 可靠. 此外，高度扭曲的分子几何结构不仅抑制了聚集 引起的发射猝灭, 而且阻止了位于中心接受电子的苯甲
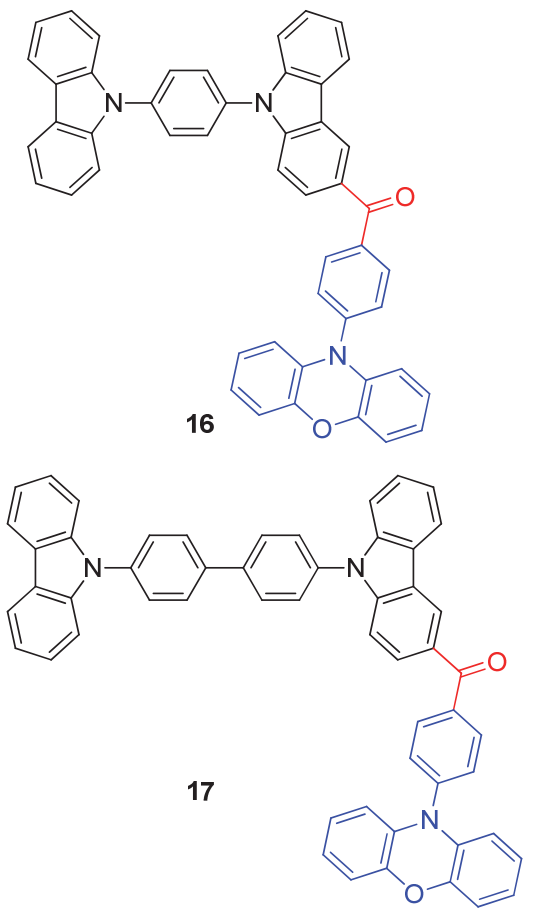

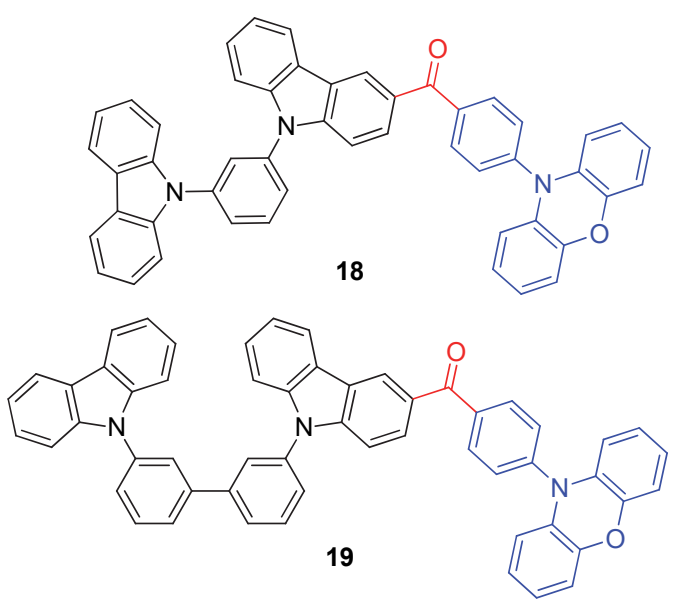

酰部分的激子短程 Dexter 能量转移, 从而提高了器件的 发光效率和高亮度下的稳定性.

以上文献证明双极性载流子传输材料与 AIDF 部分 相结合是一种可行的策略, 2020 年, 赵祖金课题组 ${ }^{[46]}$ 利 用 Bz 作受体, PXZ 作给体, 将二者接枝到双极性载流子 传输材料上, 设计了 20 和 21 . 其 $\Delta E_{\mathrm{ST}}$ 分别为 0.021 和 $0.013 \mathrm{eV}$, 纯膜中的 PLQY 分别为 $66.5 \%$ 和 $67.9 \%$, 具有 明显的延迟寿命, 分别为 1.58 和 $1.68 \mu \mathrm{s}$. 非掺杂器件结 构为 ITO/HATCN $(5 \mathrm{~nm}) /$ TAPC $(30 \mathrm{~nm}) /$ TCTA $(5 \mathrm{~nm}) /$ EML $(20 \mathrm{~nm}) / \operatorname{TmPyPB}(50 \mathrm{~nm}) / \mathrm{LiF}(1 \mathrm{~nm}) / \mathrm{Al}$, 基于 $\mathbf{2 0}$ 的器件获得最好的 $\mathrm{EL}$ 性能, 最大 CE、PE 和 $\mathrm{EQE}$ 分别 为 $57.6 \mathrm{~cd} \cdot \mathrm{A}^{-1}, 49.7 \mathrm{~lm} \cdot \mathrm{W}^{-1}$ 和 $17.3 \%$. 在不同的电压下, 在 EL 光谱中没有观察到载流子传输材料片段(35DCZPP 和 26DCZPP)的发射, 说明其光谱十分稳定. 且因 为引入了双极性载流子传输基团, 两个器件都显示出极 高的效率稳定性, 基于 $\mathbf{2 0}$ 的器件在 1000、5000、10000 $\mathrm{cd} \cdot \mathrm{m}^{-2}$ 的亮度下表现出的效率滚降分别为 $0.6 \%, 7.5 \%$, $16.2 \%$. 这些结果进一步验证了组合 AIDF 基团与双极 载流子传输基团的分子设计策略的可行性.

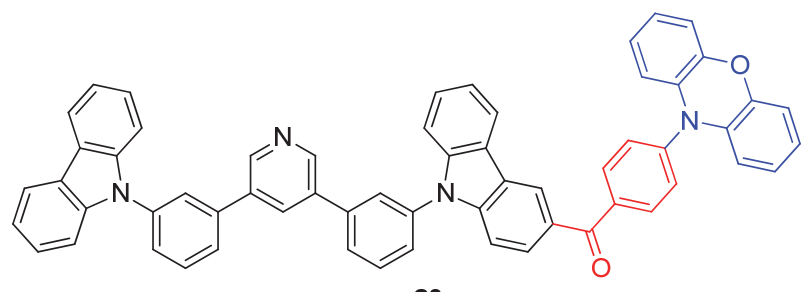

20

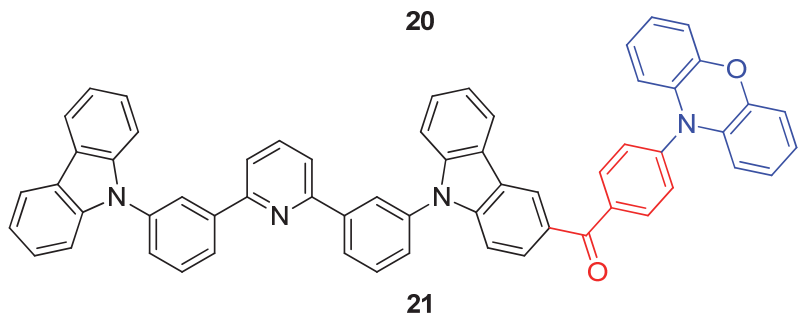

21

最近, 本课题组 ${ }^{[47]}$ 设计合成了两个具有双重发射
的 AIE 分子 $\mathbf{2 2}$ 和 $\mathbf{2 3}$, 分子内 $\mathrm{TADF}$ 和分子间 TADF/室 温磷光(room-temperature phosphorescence, RTP)同时出 现，显示出双发射带的白光发射，在制造高显色指数的 白光 OLED 上显示出巨大潜力. 在纯 THF 溶液中, 分子 22 在 404 和 $430 \mathrm{~nm}$ 处显示双峰, 23 在 $476 \mathrm{~nm}$ 处观察到 红移的无精细振动峰, 且两个分子都在溶液中表现出典 型的溶剂化发光变色效应, 表明其具有 ICT 性质. 在 $\mathrm{THF}$ 和水的混合溶液中，当含水量体积分数小于 $50 \%$ 时, 两个分子的 PL 强度在减弱的同时发生了红移, 这 是典型的 TICT, 但当含水量超过 $50 \%$ 后, 两个分子表现 出 AIE 性质, PL 强度大大增强. 在瞬态 PL 光谱的测量 中，两个分子的磷光寿命随着温度的升高而降低，而 23 有着延迟苂光, 其比例随温度的升高而增加. $22 、 23$ 的 $\Delta E_{\mathrm{ST}}$ 分别为 $0.262 、 0.264 \mathrm{eV}$, 固态 PLQY 为 $30.4 \%$ 、 $45.2 \%$, 延迟寿命为 $2.4 、 4.0 \mu \mathrm{s}$. 非掺杂器件结构为 ITO/TAPC (30 nm)/mCP (10 nm)/EML (25 nm)/3TPYMB $(45 \mathrm{~nm}) / \mathrm{LiF}(1 \mathrm{~nm}) / \mathrm{Al}(100 \mathrm{~nm})$, 两种器件都在 460 和 $600 \mathrm{~nm}$ 处显示双 $\mathrm{EL}$ 发射峰, 基于 22 和 23 的器件发出 的白光分别有 88.80 和 89.05 的高显色指数.
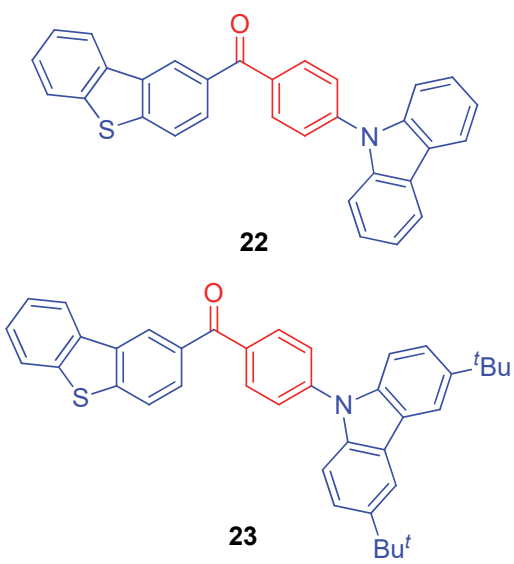

我们课题组 ${ }^{[48]}$ 也利用自主研发的新型含氮杂环的 二芳基酮受体设计了三个分子 $24 \sim 26$, 扭曲的构象使 其具有较低的 $\Delta E_{\mathrm{ST}}$, 分别为 $0.045 、 0.078$ 和 $0.27 \mathrm{eV}$. 在 纯 THF 溶液中, 三个分子荧光微弱, PLQY 也较低, 分 别为 $3.2 \%, 1.2 \%$ 和 $8.8 \%$, 表现出纳秒级的荧光寿命, 观 察不到延迟苂光. 而在纯膜中, 苂光强度显著增强, 延 迟寿命明显增加(分别为 $0.8 、 0.7$ 和 $1.7 \mu \mathrm{s}$ ), 固态 PLQY 提升较大(分别为 $55.8 \% 、 38.2 \% 、 13.5 \%$ ), 表现出 AIDF 性质. 非掺杂器件结构为 ITO/HATCN (5 nm)/TAPC (25 $\mathrm{nm}) / \mathrm{TCTA}(5 \mathrm{~nm}) / \mathrm{EML}(35 \mathrm{~nm}) / \mathrm{TmPyPB}(55 \mathrm{~nm}) / \mathrm{LiF}(1$ $\mathrm{nm}) / \mathrm{Al}$, 基于 $\mathbf{2 4}$ 的器件获得最好的 $\mathrm{EL}$ 性能, 分别为 $19.86 \mathrm{~cd} \cdot \mathrm{A}^{-1}, 17.35 \mathrm{~lm} \cdot \mathrm{W}^{-1}$ 和 $7.04 \%$ 的 $\mathrm{EQE}$, 在 1000 $\mathrm{cd} \cdot \mathrm{A}^{-1}$ 下的效率滚降仅为 $2.3 \%$, 效率稳定性高. 

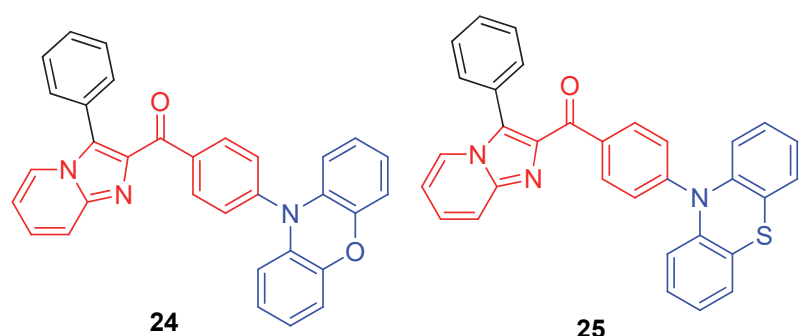

25

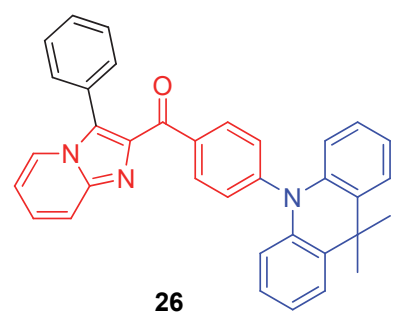

为进一步探索该二芳基酮受体体系, 在 24 的基础 上通过在受体不同位置引入㲵基的策略设计了分子 27 和 28, 以研究分子结构与性质的关系 ${ }^{[49]}$. 两个分子 $\Delta E_{\mathrm{ST}}$ 分别为 0.03 和 $0.02 \mathrm{eV}$, 在 THF 溶液中测得 PLQY 分别为 $0.51 \%$ 和 $1.14 \%$, 而在纯膜中 PLQY 分别为 $31.48 \%$ 和 $32.32 \%$, 延迟寿命为 0.79 和 $0.52 \mu \mathrm{s}$. 氰基的 引入增强了二芳基酮受体的吸电子能力, LUMO 进一步 降低并均匀分散于受体部分, 分子共轭程度增加, 发光 红移，在聚集态时 27、28 显示出 580 和 $589 \mathrm{~nm} \mathrm{(24}$ 为 $544 \mathrm{~nm}$ )的红光发射. 氰基的引入也使分子内部 CT 过程 增强, 一定程度上抑制了 TTA, 器件效率能保持相对稳 定. 对比上述三个分子, 我们发现氰基的引入能加强 $\mathrm{S}_{1}$ 和 $\mathrm{T}_{1}$ 轨道的耦合, 提高 RISC 速率, 一定程度上缩短了 延迟寿命, 这对于 TADF 器件的制造是有利的. 非掺杂 器件结构为 ITO/TAPC (25 nm)/emitter (35 nm)/TmPyPB $(55 \mathrm{~nm}) / \mathrm{LiF}(1 \mathrm{~nm}) / \mathrm{Al}$, 基于 $\mathbf{2 7}$ 和 $\mathbf{2 8}$ 的器件获得更好的 $\mathrm{EL}$ 性能, 最大 $\mathrm{CE} 、 \mathrm{PE} 、 \mathrm{EQE}$ 分别为 $7.15 \mathrm{~cd} \bullet \mathrm{A}^{-1}, 2.20 \mathrm{~lm} \bullet$ $\mathrm{W}^{-1}, 4.94 \%$.

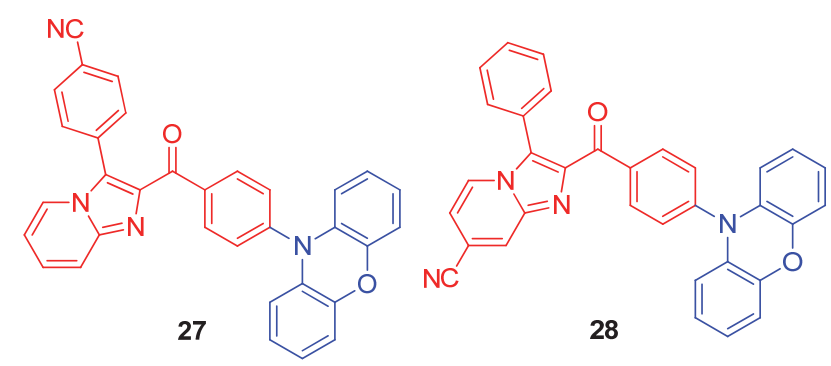

基于真空蒸镀的 AIDF-OLED 取得很大进展, 但是 基于溶液加工型 AIDF-OLED 仍然处于空白. 为探究 AIDF 分子在溶液加工 OLED 上的应用, 2019年, 赵祖金 课题组 ${ }^{[50]}$ 通过将 $\mathrm{Bz}$ 接入由长链烷基取代的 $\mathrm{Cz}$ 中设计一 个新的受体, 以便提高它们在溶液处理过程中的成膜能
力. 基于该受体的两个长链烷基 AIDF 小分子 $\mathbf{2 9}$ 和 $\mathbf{3 0}$ 由于给体和苯环近乎垂直的二面角表现出高度扭曲构 象, $\Delta E_{\mathrm{ST}}$ 分别低至 0.02 和 $0.04 \mathrm{eV}$. 纯膜中的 PLQY 分别 为 $38.3 \% 、 59.5 \%$, 延迟寿命为 $1.2 、 2.9 \mu \mathrm{s}$. 以 $\mathrm{ITO} /$ PEDOT:PSS (50 nm)/PVK (30 nm)/EML/TmPyPB (40 $\mathrm{nm}) / \mathrm{LiF}(1 \mathrm{~nm}) / \mathrm{Al}$ 为结构制备出系列器件. 基于 29 和 30 的非掺杂 OLED 表现出小的效率滚降(在 $5000 \mathrm{~cd} \cdot \mathrm{A}^{-2}$ 下 的效率下降为 $2.8 \% \sim 9.6 \%$ ). 在掺杂器件中, 基于以 29 $(w=10 \%): \mathrm{CBP}$ 组成的 EML (50 nm) 获得了最好的 EL 性 能, 最大 $\mathrm{CE} 、 \mathrm{PE}$ 和 $\mathrm{EQE}$ 分别为 $37.62 \mathrm{~cd} \bullet \mathrm{A}^{-1}, 17.76$ $1 \mathrm{~m} \cdot \mathrm{W}^{-1}$ 和 $12.13 \%$. 这对溶液加工 OLED 而言是一个明 显的进步，因为高电压下的高激子浓度往往会通过双分 子猝灭过程(TTA、STA 等)引起激子湮没. 这些过程通 常涉及分子之间的短程 Dexter 能量转移(DET). 在这种 情况下，通过延长激子之间的距离，可以有效地减少高 浓度的激子湮灭. 对于分子 29 和 30 , 其激子主要位于 中心羰基单元上，因为它的能级较低. 这两种化合物都 是高度扭曲的, 在聚合状态下采用松散的堆积方式, 可 以防止激子相互靠近，从而大大降低了激子湮灭带来的 效率滚降.

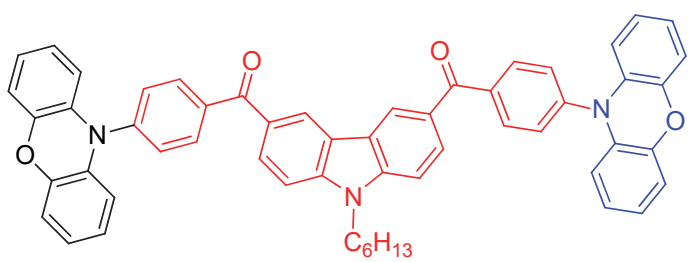

29

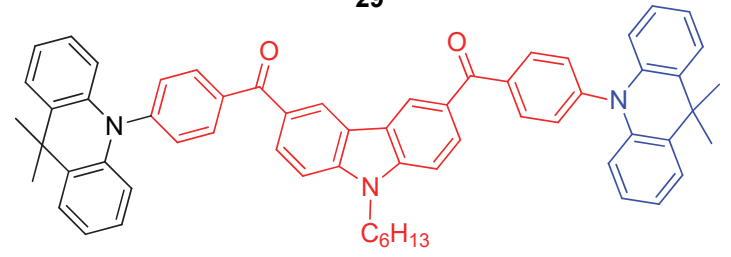

30

同年, 为了探究溶液加工 AIDF-OLED 器件, 王利 祥课题组 ${ }^{[51]}$ 设计了两个荧光分子 31 和 32 , 由于大平面 刚性的给体 TAT 和受体 BP 之间高度扭曲的构型以及大 的二面角, 有利于 HOMO 和 LUMO 的分离, 两者都具 有小的 $\Delta E_{\mathrm{ST}}$ (分别为 $0.06 、 0.09 \mathrm{eV}$ ), 并在纯膜中表现出 高的 PLQY(分别为 $51 \% 、 44 \%$ ), 得益于额外的芳酮单元 的加入, 分子 32 具有更短的延迟寿命 $(0.54 \mu \mathrm{s})$, 有利于 抑制 STA 和 TTA 的猝灭过程. 非掺杂器件结构为 ITO/ PEDOT:PSS (25 nm)/EML (25 nm)/TmPyPB (55 nm)/LiF $(1 \mathrm{~nm}) / \mathrm{Al}$, 基于 32 的器件获得了更好的 $\mathrm{EL}$ 性能, 分别 为 $20.9 \mathrm{~cd} \cdot \mathrm{A}^{-1}, 21.8 \mathrm{~lm} \cdot \mathrm{W}^{-1}$ 以及 $9.8 \%$ 的 $\mathrm{EQE}$ ，在 2000 $\mathrm{cd} \cdot \mathrm{m}^{-2}$ 下效率滚降仅为 $2 \%$. 作者认为这是由于引入更 多的 BP 受体单元而改善了电子与空穴的传输速率，导 
致 EML 中的载流子传输更加平衡, 电子与空穴的复合 率提高, 从而提高了器件的 $\mathrm{EQE}$ 与稳定性.

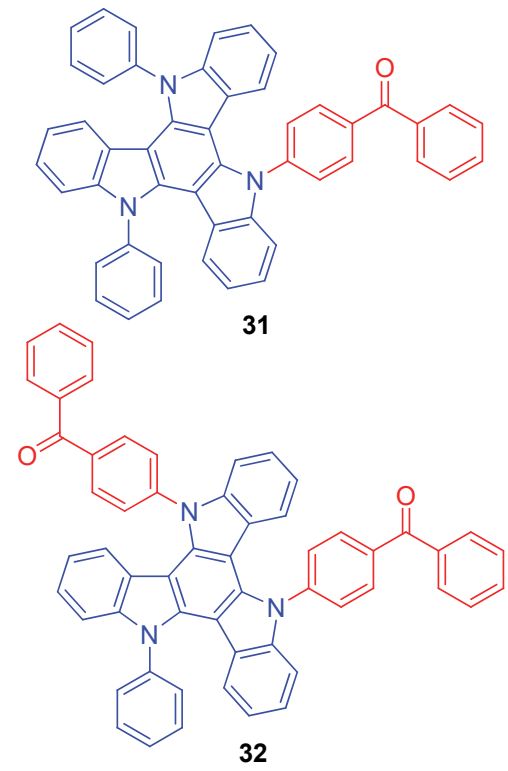

2020 年, 祁争健课题组 ${ }^{[52]}$ 利用 BP、DMAC、9,9二苯基-9,10-二氢呋啶、 PXZ 以及三苯胺设计合成了一 系列树状与非树状的新型苂光分子(分子 33 38). 以三 苯胺为中心, 引入近似正交的三元结构的树状分子拥有 更扭曲的构型, $\Delta E_{\mathrm{ST}}$ 更小 $(0.04 \sim 0.07 \mathrm{eV})$, 能进行更有 效的 RISC. 此外, 树枝状大分子的对称几何结构通过 增加多个跃迁通道的跃迁积分, 有利于增大辐射跃迁速 率常数. 且由于聚集态下分子间 $\pi-\pi$ 相互作用得到抑制, 它们的固态 PLQY 值更高, 36、37、38 的 PLQY 分别为 $46.1 \% 、 64.8 \% 、 66.5 \%$. 六个分子都具有较长的延迟苂 光, 在 $0.8 \sim 1.7 \mu \mathrm{s}$ 之间, 但树状分子 RISC 速率与辐射 跃迁速率都高于非树状分子, 表现出更快的 TADF 过程. 非掺杂器件结构为 ITO/PEDOT:PSS $(40 \mathrm{~nm}) /$ EML (40 $\mathrm{nm}) / \mathrm{TPBi}(30 \mathrm{~nm}) / \mathrm{Cs}_{2} \mathrm{CO}_{3}(2 \mathrm{~nm}) / \mathrm{Al}(100 \mathrm{~nm})$, 基于 $\mathbf{3 5}$ 的器件获得最好的 EL 性能, 最大 CE、PE、 $\mathrm{EQE}$ 分别 为 $13.0 \mathrm{~cd} \cdot \mathrm{A}^{-1}, 7.0 \mathrm{~lm} \cdot \mathrm{W}^{-1}$ 和 $4.3 \%$. 以树状分子制造的 器件具有更高的 $\mathrm{EL}$ 性能, 基于 $\mathbf{3 8}$ 的器件最大 CE、PE、 $\mathrm{EQE}$ 分别为 $37.2 \mathrm{~cd} \cdot \mathrm{A}^{-1}, 14.6 \mathrm{~lm} \cdot \mathrm{W}^{-1}$ 和 $12.1 \%$. 值得一 提的是, 由于树状分子中引入了支链烷基而增强它们的 溶解性, 可以旋涂形成高质量且无针孔的均匀薄膜, 这 些分子在溶液加工制备大面积 OLED 上显示出巨大潜 力.

\section{2 二苯砜及其衍生物}

二苯甲酮类分子由于结构调控上的简易快捷而受 到广泛关注, 且由于引入 AIE 而克服了因系间窝跃速率 大于苂光速率而导致的荧光量子产率较低 ${ }^{[53]}$ 的缺点, 但其形成的 D-A-D'型分子扭转角并不大, 可能会在一

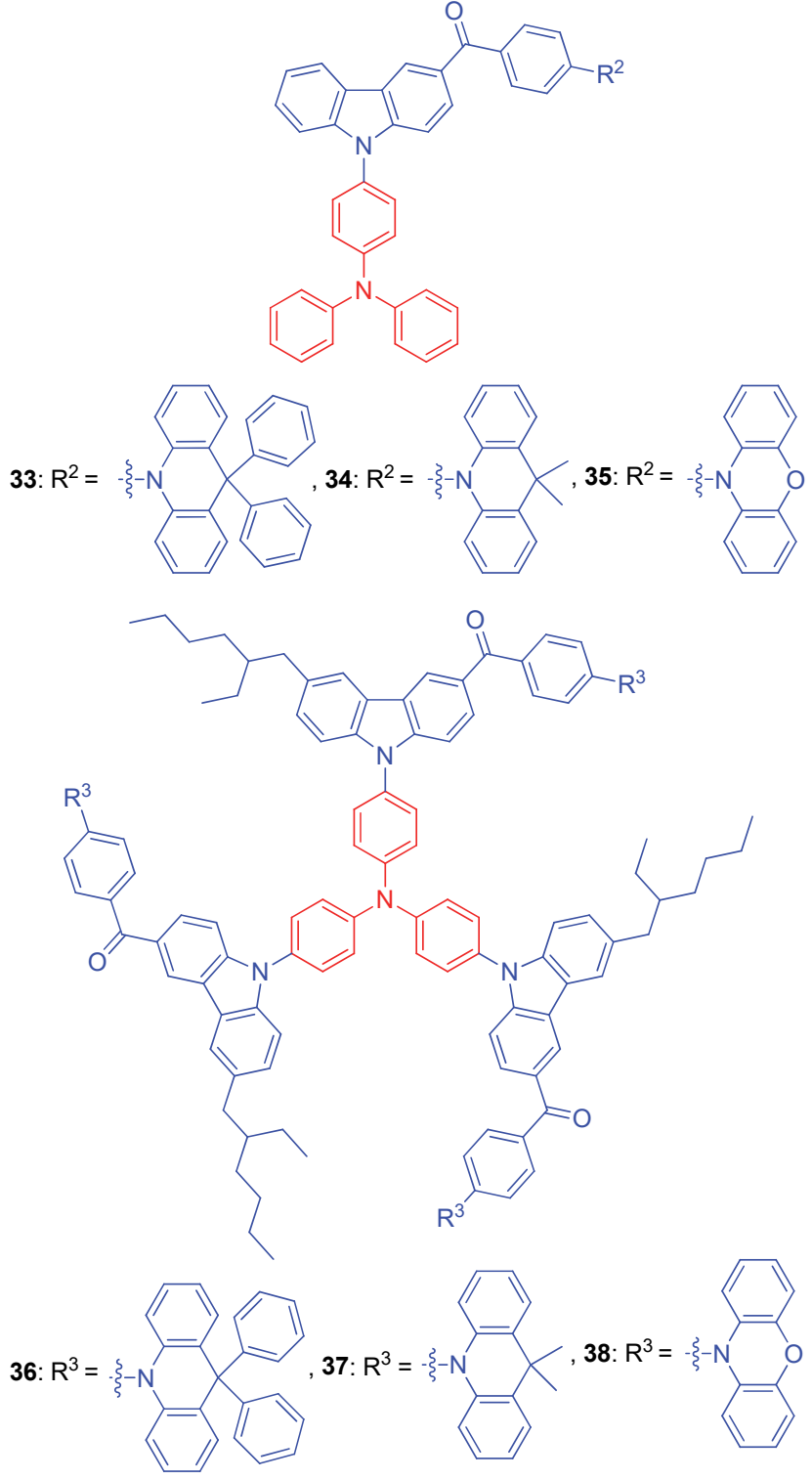

定程度上限制 HOMO、LUMO 的分离和获得小的 $\Delta E_{\mathrm{ST}}$, 从而抑制 AIDF. 不同于二苯甲酮，二苯砜基团的中心 是一个磺酰基, 基团中的两个 $\mathrm{O}$ 原子赋予它较强的吸电 子能力(Scheme 2). 同时, 因其具有四面体的几何构型, 将不同给体连接上苯环, 形成的给受体间的扭转角将更 大, 在一定程度上阻断了两端的 $\pi-\pi$ 共轭，抑制激子猝 灭，因此在 AIDF 分子设计上也颇受关注. 基于二苯砜 及其衍生物的相关性质见表 2 .

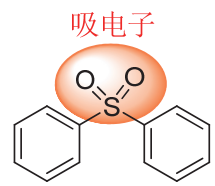

连接给体 $\mathrm{D}$

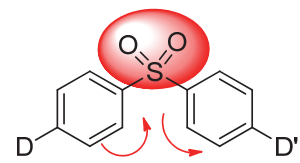

阻断共轭 扭转角变大
图式 2 二苯砜类 AIDF 分子设计策略

Scheme 2 Molecular design strategy of diphenylsulfone-based AIDF compounds 
表 2 二苯砜类 AIDF 分子在溶液中或分散在主体薄膜或非掺杂成膜中光致发光波长 $(\lambda \mathrm{PL})$ 、苂光量子产率(PLQY), 纯膜中荧光寿 命 $\left(\tau_{\mathrm{PF}}, \tau_{\mathrm{DF}}\right)$ 、单重态 - 三重态能级差 $\left(\Delta E_{\mathrm{ST}}\right)$, 非掺杂或掺杂器件的电致发光波长 $\left(\lambda_{\mathrm{EL}}\right)$ 、最大外量子效率 $(\mathrm{EQE})$ 以及 $\mathrm{CIE}$ 色坐标

Table 2 PL peak $\left(\lambda_{\mathrm{PL}}\right)$ in solution or dispersed in a host film or in neat film, photoluminescence quantum yield (PLQY), lifetime in neat film $\left(\tau_{\mathrm{PF}}, \tau_{\mathrm{DF}}\right)$, singlet-triplet energy gap $\left(\Delta E_{\mathrm{ST}}\right)$ of diphenylsulfone-AIDF, EL peak $\left(\lambda_{\mathrm{EL}}\right)$ and maximum EQE, CIE for nondoped or doped devices, respectively

\begin{tabular}{|c|c|c|c|c|c|c|c|c|c|}
\hline Compd. & $\lambda_{\mathrm{PL}} / \mathrm{nm}$ & PLQY/\% & $\tau_{\mathrm{PF}} / \mathrm{ns}$ & $\tau_{\mathrm{DF}} / \mu \mathrm{s}$ & $\Delta E_{\mathrm{ST}} / \mathrm{eV}$ & $\lambda_{\mathrm{EL}} / \mathrm{nm}$ & EQE/\% & CIE & Ref. \\
\hline 39 & $-/ 547$ & $9.9 / 10.4$ & - & - & 0.09 & - & $-/ 1.1$ & $-/(0.46,0.43)$ & [54] \\
\hline 40 & $-/ 542$ & $16.1 / 23.5$ & - & - & 0.06 & - & $-/ 4.5$ & $-/(0.45,0.40)$ & [54] \\
\hline 41 & $-/ 535$ & $15.9 / 19.5$ & - & - & 0.04 & - & $-/ 10.4$ & $-/(0.37,0.38)$ & [54] \\
\hline 42 & $537 / 523$ & $0.8 / 1.0$ & - & - & 0.14 & - & $-/ 14$ & $-/(0.35,0.58)$ & [55] \\
\hline 43 & $566 / 530$ & $25 / 55$ & - & - & 0.18 & $-/ 532$ & $-/ 15.4$ & $-/(0.40,0.54)$ & [55] \\
\hline 44 & $556 / 522$ & $16 / 69$ & - & - & 0.03 & $-/ 540$ & $-/ 16.3$ & $-/(0.37,0.55)$ & [55] \\
\hline 45 & $486 / 512$ & $68.8 / 61.5$ & 16.1 & 2.49 & 0.02 & $504 / 500$ & $16.4 / 16.3$ & $\begin{array}{c}(0.27,0.50) / \\
(0.24,0.44)\end{array}$ & [56] \\
\hline 46 & $-/ 520$ & $0.9 / 91.9$ & 4.4 & 19.1 & 0.32 & $524 /-$ & $28.7 /-$ & - & [57] \\
\hline 47 & $-/ 530$ & $-/ 97.3$ & 6.3 & 62.2 & 0.25 & $518 /-$ & $20.7 /-$ & - & [57] \\
\hline 48 & $516 / 502$ & $0.5 / 59$ & - & 3.2 & 0.02 & $-/ 504$ & $-/ 13$ & $-/(0.23,0.43)$ & [58] \\
\hline 49 & $505 / 499$ & $0.41 / 65$ & - & 3.8 & 0.03 & $-/ 498$ & $-/ 12.8$ & $-/(0.20,0.39)$ & [58] \\
\hline 50 & $464 / 476$ & $0.53 / 77$ & - & 5.4 & 0.09 & $-/ 480$ & $-/ 20.5$ & $-/(0.17,0.29)$ & [58] \\
\hline
\end{tabular}

2017 年，间寿科课题组 ${ }^{[54]}$ 利用两种不同颜色的荧 光基团合成了一系列共聚物 39 41. 尽管其 AIE 性质并 不显著, 苂光量子产率从甲苯溶液到薄膜提高不多, 但 制备出的共聚物能通过调节两种基团的比例而改变发 光波长, 其中分子 41 实现了暖白光的发射, 作者认为通 过将具有高能隙和低能隙的不同基团进行共聚是一种 实现白光发射的有效手段. 结构为 ITO/PEDOT:PSS (40 $\mathrm{nm})$ polymer:TCTA:TAPC $(10 ： 65: 25$, 质量分数之 比) $(45 \mathrm{~nm}) / \operatorname{TmPyPB}(50 \mathrm{~nm}) / \mathrm{LiF}(0.8 \mathrm{~nm}) / \mathrm{Al}(80 \mathrm{~nm})$ 的 掺杂器件中, 41 取得最好的 EL 性能, 最大 CE、PE、EQE 分别为 $23.0 \mathrm{~cd} \cdot \mathrm{A}^{-1}, 32.8 \mathrm{~lm} \cdot \mathrm{W}^{-1}$ 以及 $10.4 \%$. 这是报道 的首例暖白色 TADF 聚合物 OLED, 也证明了 AIE 效应 在调控聚合物分子发光波长的可行性.

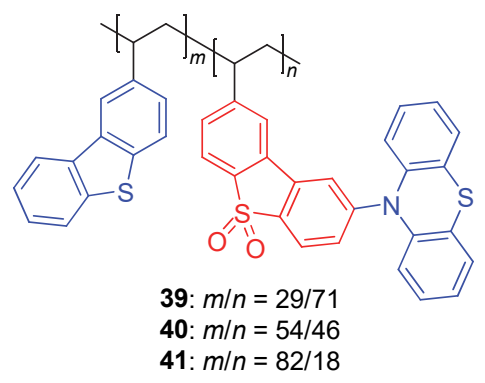

位置异构被证明是一种简单而有效的调节发光分 子发光特性的方法. 在之前的工作中, 证明了通过位置 异构策略, 无论是真空蒸镀器件还是溶液加工器件, 都 可以实现高 PLQY 和高 EL 效率. 王鹰课题组 ${ }^{[55]}$ 利用噻 吨酮(Thioxanthone, TXO)和 $N$-苯基咔唑合成了三个分 子异构体 42、43、44, 研究 $N$-苯基咔唑的不同取代位置 导致不同的性质. 42 由于 $\mathrm{V}$ 形结构降低了共轭程度, 无
法进行有效的电子云跃迁导致其振子强度较低, 在纯膜 和掺杂膜的 PLQY 都不高; 3 号位取代的 41 由于电子云 重叠较大导致大的 $\Delta E_{\mathrm{ST}}, 43$ 和 44 在掺杂膜下的苂光量 子产率比纯膜中有了显著提高，分别为 $55 \%$ 、 $69 \%$. 与 43 不同, 4 号位取代的 44 由于强的自旋轨道耦合, 在 $300 \mathrm{~K}$ 下表现出显著的磷光发射. 基于 ITO/HAT-CN (5 $\mathrm{nm}) / \mathrm{TAPC}(40 \mathrm{~nm}) / \mathrm{mCP}(10 \mathrm{~nm}) / \mathrm{PPO} 21: 42(w=6 \%)(20$ $\mathrm{nm}) / \mathrm{TmPyPB}(45 \mathrm{~nm}) / \mathrm{LiF}(0.9 \mathrm{~nm}) / \mathrm{Al}$ 的器件最大 CE、 $\mathrm{PE} 、 \mathrm{EQE}$ 分别为 $49.2 \mathrm{~cd} \cdot \mathrm{A}^{-1}, 47.7 \mathrm{~lm} \bullet \mathrm{W}^{-1}$ 和 $16.3 \%$.
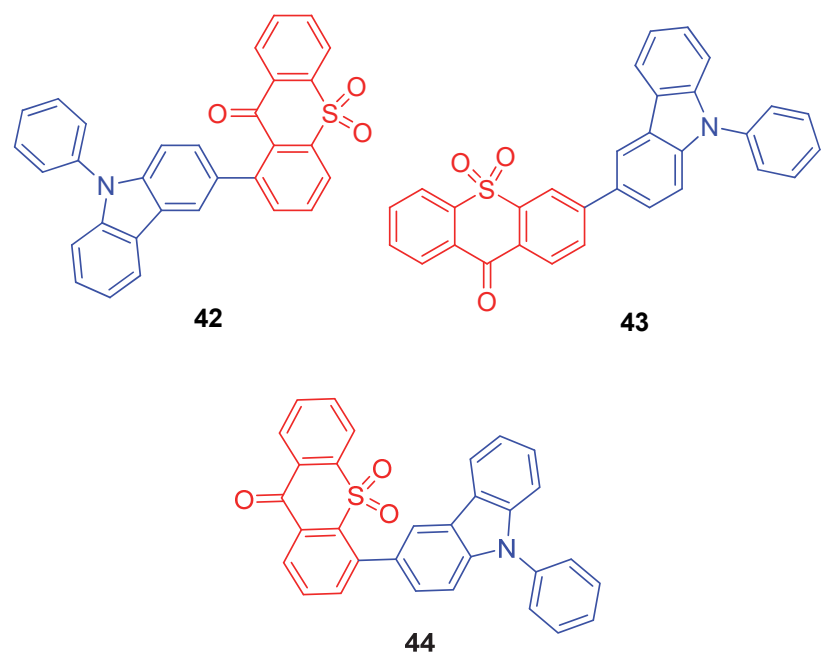

2018 年, 王雷课题组 ${ }^{[56]}$ 以吩噻嗪氧化物为受体, 以 吩噁嗪为给体设计出分子 45 . 因分子内存在两个近乎 垂直的二面角, 使该分子呈现高度扭曲的构型, 形成低 至 $0.02 \mathrm{eV}$ 的 $\Delta E_{\mathrm{ST}}$. 在 $w=80 \%$ 的掺杂膜和纯膜中, PLQY 分别为 $68.75 \%$ 和 $61.54 \%$ ，延迟寿命为 2.61 和 2.49 
$\mu$ s. 在结构为 $\mathrm{ITO} / \mathrm{MoO}_{3}(10 \mathrm{~nm}) / \mathrm{TAPC}(40 \mathrm{~nm}) / \mathrm{mCP}(20$ $\mathrm{nm}) / \mathrm{EML}(30 \mathrm{~nm}) / \mathrm{DPEPO}(20 \mathrm{~nm}) / \mathrm{TPBi}(15 \mathrm{~nm}) / \mathrm{LiF}(1$ $\mathrm{nm}) / \mathrm{Al}(100 \mathrm{~nm})$ 的非掺杂器件中, 最大 CE、PE、EQE 分别为 $44.9 \mathrm{~cd} \cdot \mathrm{A}^{-1}, 32.0 \mathrm{~lm} \cdot \mathrm{W}^{-1}$ 和 $16.4 \%$, 掺杂器件 $\mathrm{EL}$ 性能与之相当.

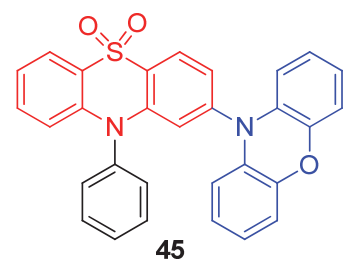

2019 年, 池振国课题组 ${ }^{[57]}$ 通过将给体和受体分别 定位于苯基的邻位和对位设计了两个分子 46 和 47 . 在 邻位取代的 46 中, 紧密的空间 D-A 相互作用使之在产 生空间共轭的同时降低了分子振动并抑制了非辐射跃 迁. 此外, 该分子还具有 TBCT 和 TSCT 双重电荷转移 途径, $\Delta E_{\mathrm{ST}}$ 分别为 0.32 和 $0.25 \mathrm{eV}$. 在薄膜中, 46 和 47 的 PLQY 达 91.9\%和 $97.3 \%$, 延时寿命分别为 $19.1 \mu \mathrm{s}$ 和 $62.2 \mu \mathrm{s}$. 非掺杂器件结构为 ITO/PEDOT:PSS $(30 \mathrm{~nm}) /$ MCP (20 nm)/EML (30 nm)/TPBI (40 nm)/LiF (1 nm)/Al (100 nm), 基于 46 的器件获得更好的 EL 性能, 最大 $C E 、 P E 、 E Q E$ 分别为 $82.3 \mathrm{~cd} \bullet \mathrm{A}^{-1}, 51.8 \mathrm{~lm} \cdot \mathrm{W}^{-1}$ 和 $28.7 \%$.
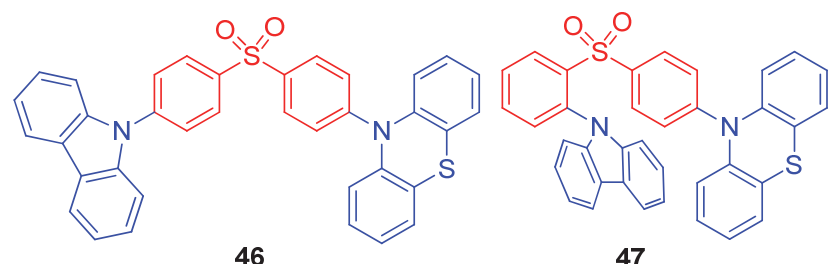

47

同样是调整给受体位置, 杨楚罗课题组 ${ }^{[58]}$ 利用 DMAC 以及二苯砜设计了三个同分异构体 48、49、50. 作者系统地研究了给受体相对位置的差异对分子性质 及器件性能的影响, 并指出取代基的微调有助于平衡小 $\Delta E_{\mathrm{ST}}$ 与高 PLQY 之间的矛盾. 由于高度扭曲的二面角, 三个分子显示出低的 $\Delta E_{\mathrm{ST}}$, 分别为 $0.02 、 0.03$ 和 $0.09 \mathrm{eV}$. 在 $w=10 \%$ 的 DPEPO 掺杂薄膜中, 其 PLQY 分别为 $59 \% 、 65 \% 、 77 \%$, 延迟寿命分别为 $3.2 、 3.8 、 5.4 \mu \mathrm{s}$. 值 得注意的是, 50 显示出较小扭转角, 且其 LUMO、 HOMO 轨道重叠程度更高, 因此, 其性能要优于其余两 个分子. 结构为 ITO/HATCN $(5 \mathrm{~nm}) / \mathrm{TAPC}(30 \mathrm{~nm}) / \mathrm{mCP}$ $(10 \mathrm{~nm}) /$ EML $(20 \mathrm{~nm}) / \mathrm{DPEPO}(10 \mathrm{~nm}) / \mathrm{TmPyPB}$ (30 $\mathrm{nm}) / \mathrm{Liq}(1.5 \mathrm{~nm}) / \mathrm{Al}(100 \mathrm{~nm})$ 的掺杂器件中, 基于 $\mathbf{5 0}$ 的 器件呈现天蓝色发射, 色坐标为 $(0.17,0.29)$, 最大 CE、 $\mathrm{PE} 、 \mathrm{EQE}$ 分别为 $40.7 \mathrm{~cd} \bullet \mathrm{A}^{-1}, 36.3 \mathrm{~lm} \bullet \mathrm{W}^{-1}$ 和 $20.5 \%$, 这 是当时器件性能较好的天蓝光 TADF-OLED. 结果表明,
这种简单的异构化策略对于探索分子结构和功能之间 的关系具有重要的指导意义, 并且可以利用这种简单的 异构化策略调控 PL 和 EL 的性质，从而开发出更高效的 AIDF 发光材料.

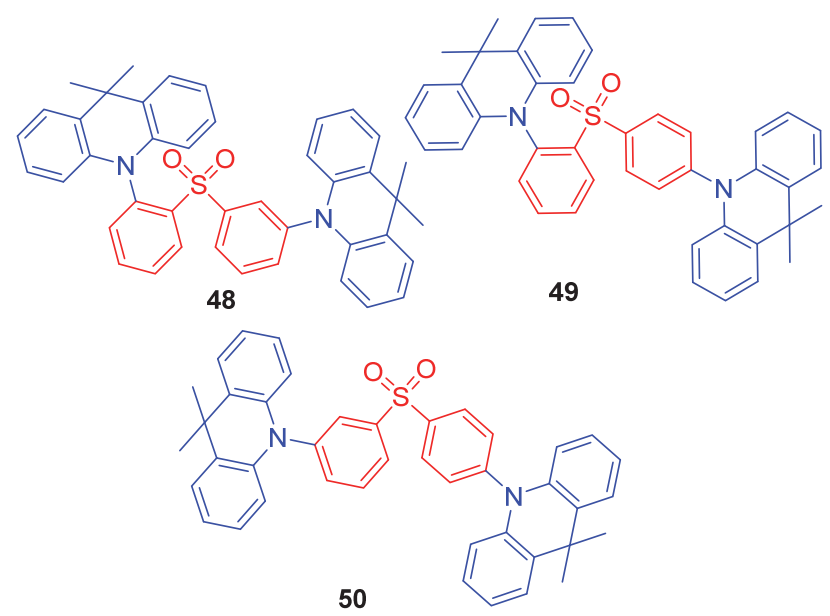

\section{3 三嗪及其衍生物}

二苯砜本身四面体的构型利于扭曲构象的形成, 并 且能够与苯环连接时产生较大的扭转二面角, 以获得较 低的 $\Delta E_{\mathrm{ST}}$ 值，但也限制了其在 HOMO、LUMO 上进一 步分离. 三嗪作为含三个 $\mathrm{N}$ 原子的芳香族化合物，它具 有缺电子的特性，因而也被用作 TADF 受体单元. 该类 分子的设计多采用 1,3,5-三嗪(1,3,5-Triazine, TRZ)及其 衍生物作受体. TRZ 具有对称的平面结构, 有三个可供 修饰的位点，容易引入不同给体进行分子调控. 不同于 二苯甲酮或二苯砜，该类分子扭曲构象的形成多依赖于 空间上的巧妙设计，由于给受体空间位置的紧密相连， 电子直接通过空间 $\pi-\pi$ 共轭进行电荷转移, 经常引入苯 基增大共轭程度(Scheme 3). 相关性质见表 3.

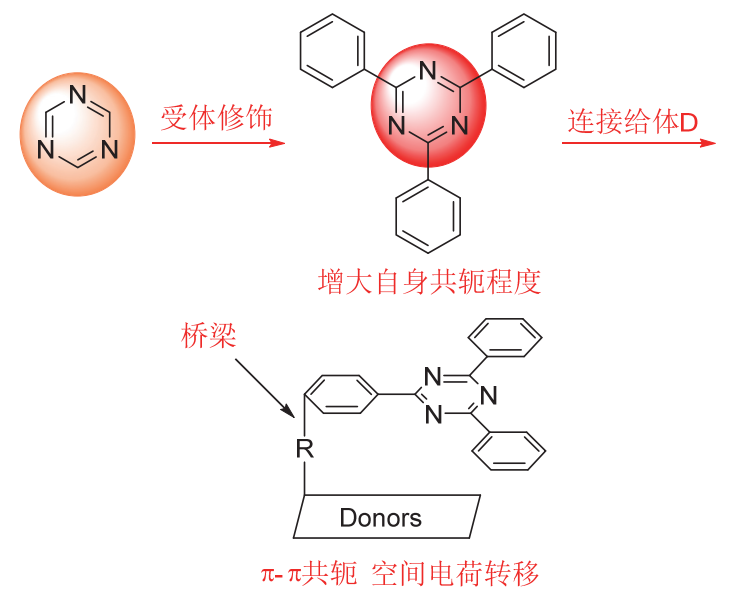

图式 3 三嗪类 AIDF 分子设计策略

Scheme 3 Molecular design strategy of triazine-based AIDF compounds 
表 3 三嗪类 AIDF 分子在溶液中或分散在主体薄膜或非掺杂成膜中光致发光波长 $\left(\lambda_{\mathrm{PL}}\right)$ 、菼光量子产率(PLQY), 纯膜中荧光寿命 $\left(\tau_{\mathrm{PF}}, \tau_{\mathrm{DF}}\right)$ 、单重态-三重态能级差 $\left(\Delta E_{\mathrm{ST}}\right)$, 非掺杂或掺杂器件的电致发光波长 $\left(\lambda_{\mathrm{EL}}\right)$ 、最大外量子效率 $(\mathrm{EQE})$ 以及 $\mathrm{CIE}$ 色坐标

Table 3 PL peak $\left(\lambda_{\mathrm{PL}}\right)$ in solution or dispersed in a host film or in neat film, photoluminescence quantum yield (PLQY), lifetime in neat film $\left(\tau_{\mathrm{PF}}, \tau_{\mathrm{DF}}\right)$, singlet-triplet energy gap $\left(\Delta E_{\mathrm{ST}}\right)$ of triazine-AIDF, EL peak $\left(\lambda_{\mathrm{EL}}\right)$ and maximum EQE, CIE for based nondoped or doped devices, respecyively

\begin{tabular}{cccccccccc}
\hline Compd. & $\lambda_{\mathrm{PL}} / \mathrm{nm}$ & $\mathrm{PLQY} / \%$ & $\tau_{\mathrm{PF}} / \mathrm{ns}$ & $\tau_{\mathrm{DF}} / \mu \mathrm{s}$ & $\Delta E_{\mathrm{ST} / \mathrm{eV}}$ & $\lambda_{\mathrm{EL}} / \mathrm{nm}$ & $\mathrm{EQE} / \%$ & $\mathrm{CIE}$ & $\mathrm{Ref.}$ \\
\hline $\mathbf{5 1}$ & $562 / 566$ & $7.7 / 66$ & - & 3.3 & 0.001 & $-/ 584$ & $-/ 10$ & - & - \\
$\mathbf{5 2}$ & $419 / 418$ & $5.9 /-$ & - & - & 0.008 & - & - & $-/ 4$ & {$[59]$} \\
$\mathbf{5 3}$ & $451 / 453$ & $6.0 / 35$ & - & 4.1 & 0.007 & $-/ 488$ & $-/ 4$ & {$[59]$} \\
$\mathbf{5 4}$ & $486 / 505$ & $20 / 54$ & - & 3.54 & 0.08 & $520 / 492$ & $3.5 / 11$ & $(0.30,0.54) /(0.22,0.42)[60]$ \\
$\mathbf{5 5}$ & $508 / 518$ & $36 / 63$ & - & 3.16 & 0.04 & $538 / 503$ & $3.1 / 14.2$ & $(0.26,0.48) /(0.25,0.47)[60]$ \\
$\mathbf{5 6}$ & $507 / 524$ & $32.8 / 47.7$ & 12.9 & 1.3 & 0.052 & $524 /-$ & $4.1 / 17.53$ & $(0.35,0.57) /(0.29,0.54)[61]$ \\
$\mathbf{5 7}$ & $515 / 525$ & $30.4 / 64.5$ & 26.8 & 1.6 & 0.069 & $532 /-$ & $11.75 / 18.43$ & $(0.36,0.58) /(0.33,0.58)[61]$ \\
$\mathbf{5 8}$ & $-/ 538$ & $-/ 67$ & 81 & 0.64 & 0.15 & $548 / 520$ & $12.8 / 22$ & $(0.42,0.55) /(0.33,0.58)[62]$ \\
$\mathbf{5 9}$ & $-/ 556$ & $-/ 42$ & 55 & 0.75 & 0.12 & $576 / 540$ & $5.9 / 13.2$ & $(0.50,0.49) /(0.40,0.56)[62]$ \\
$\mathbf{6 0}$ & $565 / 534$ & $-/ 50.5$ & - & 1.52 & 0.05 & - & - & - & - \\
$\mathbf{6 1}$ & $555 / 521$ & $-/ 48.7$ & - & 1.54 & 0.05 & - & - & {$[63]$} \\
$\mathbf{6 2}$ & $-/ 581$ & $-/ 12.8$ & - & 1.12 & 0.04 & - & - & - & {$[63]$} \\
\hline
\end{tabular}

2017 年, Tsujimoto 等 ${ }^{[59]}$ 利用 TRZ 衍生物, 以 D-A 结构，采用空间电荷转移策略设计了 3 个 $\mathrm{U}$ 型分子 51 、 52、53, 通过在中间桥连结构(9,9-二甲基杂葱)上引入不 同的给体组合，调控发射波长，并兼具 AIE 和 TADF 特 性. 在获得小的 $\Delta E_{\mathrm{ST}}(0.001 \sim 0.008 \mathrm{eV})$ 的同时，抑制了 聚集态下分子间的 $\pi-\pi$ 相互作用, 表现出 AIE 性质. 从 甲苯溶液到薄膜, 51 和 53 的 PLQY 分别从 7.7\%提高至 $65 \%, 6.0 \%$ 提高至 $35 \%$, 在掺杂膜中的延迟寿命分别为 3.3、4.1 $\mu \mathrm{s}$. 掺杂器件结构为 ITO $(100 \mathrm{~nm}) /$ TAPC (70 $\mathrm{nm}) / \mathrm{DPEPO}:$ Dopant $(w=10 \%)(30 \mathrm{~nm}) / \mathrm{DPEPO}(2 \mathrm{~nm})$ $\mathrm{TmPyPb}(45 \mathrm{~nm}) / \mathrm{LiF}(1 \mathrm{~nm}) / \mathrm{Al}$ 的器件, 基于 $\mathbf{5 1}$ 的器件 获得 $10 \%$ 的 EQE.

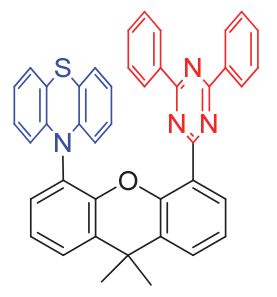

51

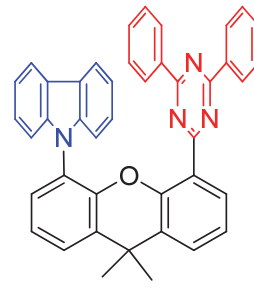

52<smiles></smiles>

53

2019 年, Wang 等[60]通过将三个 DMAC 衍生物给体 以及三个三苯基均三嗪受体引入六苯基苯, 设计了两个 新的分子 54 和 $\mathbf{5 5}$, 其 $\Delta E_{\mathrm{ST}}$ 分别为 0.04 和 $0.08 \mathrm{eV}$. 由于 空间上的紧密联系, 两个分子产生明显的空间电荷转移
现象，促进辐射跃迁的产生. 两个分子的平面非螺旋结 构使其在固态时抑制分子内运动，产生 AIE. 从纯膜到 掺杂膜，54、55 的 PLQY 分别从 20\%提至 54\%, 36\%提 至 $63 \%$, 在掺杂膜中的延迟寿命分别为 $3.54 \mu \mathrm{s}$ 和 3.16 $\mu \mathrm{s}$. 通过溶液加工的方法制备了结构为 ITO/PEDOT: PSS (40 nm)/EML (40 nm)/TSPO1 (8 nm)/TmPyPB (42 $\mathrm{nm}) / \mathrm{LiF}(1 \mathrm{~nm}) / \mathrm{Al}(100 \mathrm{~nm})$ 的器件, 使用 Ac6:54 (10 $\mathrm{wt} \%$ ) 为发射层的掺杂器件显示出最好的 EL 性能, 其 $\mathrm{EQE}$ 为 $14.2 \%$, 是当时溶液加工制备性能最好的器件之 一, 再一次显示出 AIDF 材料在溶液加工器件的巨大潜 力.<smiles>[R]c1c([R])c([R])c([R])c([R])c1[R]</smiles>

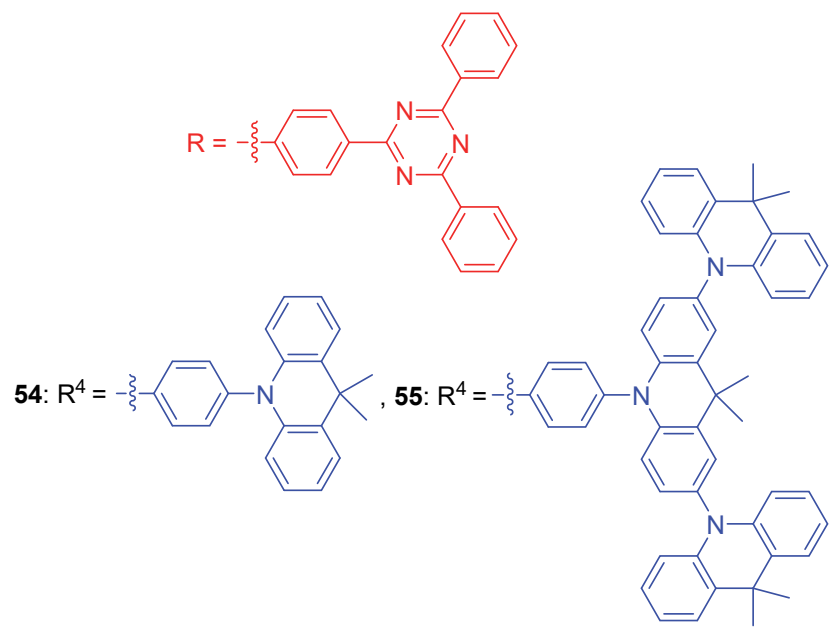

2018 年, Choi 等 ${ }^{[61]}$ 利用三苯基均三嗪作受体，鲐啶 衍生物作供体合成了两个 “三臂” 发光体 56 和 57. 其 
$\Delta E_{\mathrm{ST}}$ 分别为 0.052 和 $0.069 \mathrm{eV}$, 从溶液到薄膜, PLQY 值 分别由 $32.8 \%$ 增至 $47.7 \%$, 由 $30.4 \%$ 增至 $64.5 \%$. 在薄膜 中具有明显的延迟荧光, 分别为 1.3 和 $1.6 \mu \mathrm{s}$. 利用溶液 加工制备了非掺杂器件, 结构为 ITO/PEDOT:PSS (50 $\mathrm{nm}) / \mathrm{PVK}(30 \mathrm{~nm}) / \mathrm{EML}(35 \mathrm{~nm}) / \mathrm{TPBi}(40 \mathrm{~nm}) / \mathrm{LiF}(0.8$ $\mathrm{nm}) / \mathrm{Al}(100 \mathrm{~nm})$, 其中, 57 经混合溶液处理后获得最好 的 $\mathrm{EL}$ 性能, 最大 $\mathrm{CE} 、 \mathrm{PE} 、 \mathrm{EQE}$ 分别为 $39.31 \mathrm{~cd} \cdot \mathrm{A}^{-1}$, $27.45 \mathrm{~lm} \cdot \mathrm{W}^{-1}$ 和 $11.75 \%$. 此外, 还将两个分子掺入 $\mathrm{mCP}$ 中制备了掺杂器件, $\mathbf{5 7}$ 的 $\mathrm{EQE}$ 提升至 18.43\%.
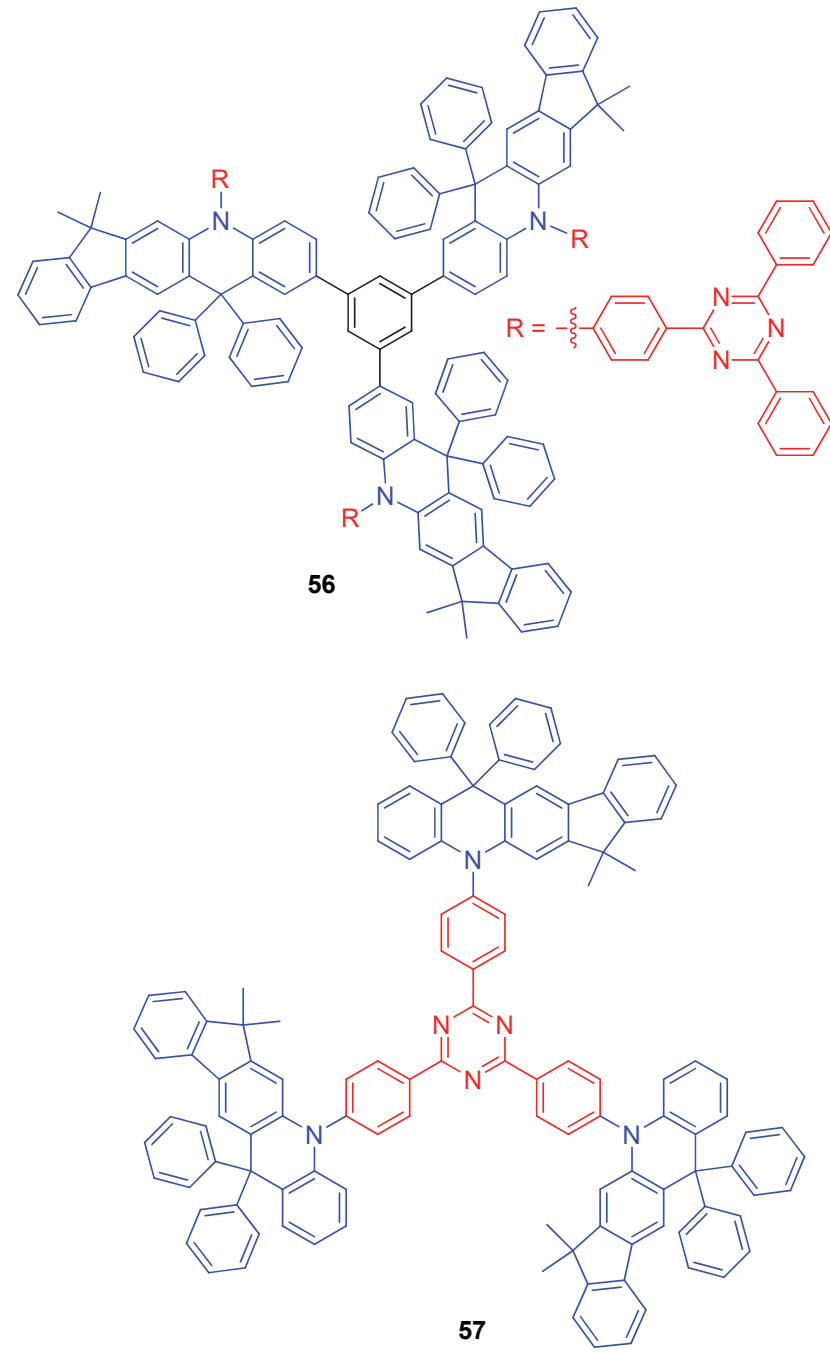

王雷课题组 ${ }^{[2]}$ 用呋啶一咔唑杂化作为供体, 嘧啶或 三苯基均三嗪作受体设计了两个分子 58、59. 其 $\Delta E_{\mathrm{ST}}$ 分别为 0.15 和 $0.12 \mathrm{eV}$, 在非掺杂膜中的 PLQY 为 $67 \%$ 和 $42 \%$, 延迟寿命为 0.64 和 $0.75 \mu \mathrm{s}$. 实验结果表明, AIDF 发射体的 AIE 特性可以通过受体种类的选择来调 节, 以三嗪为受体的分子 59 具有更显著的 AIE 效应. 器 件结构为 $\mathrm{ITO} / \mathrm{MoO}_{3}(10 \mathrm{~nm}) / \mathrm{TAPC} / \mathrm{TCTA} / \mathrm{EML} / \mathrm{TmPy}-$ $\mathrm{PB} / \mathrm{LiF}(1 \mathrm{~nm}) / \mathrm{Al}$, 由于更加突出的 $\mathrm{AIE}$ 性质, 基于 $\mathbf{5 8}$ 的器件 EL 性能优于 $\mathbf{5 9}$, 在掺杂器件中, 它的最大 CE 和 $\mathrm{EQE}$ 分别为 $73.3 \mathrm{~cd} \cdot \mathrm{A}^{-1}$ 和 $22.6 \%$, 在非掺杂器件中为
$45.2 \mathrm{~cd} \cdot \mathrm{A}^{-1}$ 和 $14.1 \%$.
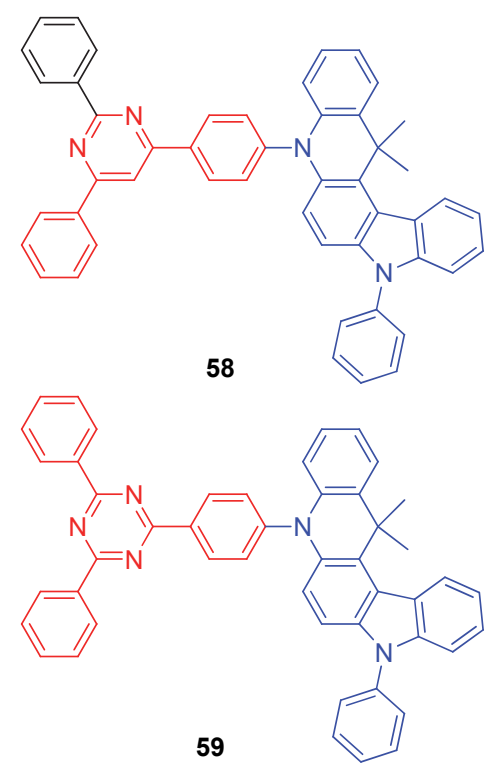

陈传峰课题组 ${ }^{[63]}$ 利用草酸和 TRZ 衍生物设计了三 个分子 60、61、62. 它们显示出小的 $\Delta E_{\mathrm{ST}}(0.04 \sim 0.05$ $\mathrm{eV})$. 在水和四氢呋喃的混合介质中, 它们表现出 AIE 性质; 纯膜下三个分子都具有微秒级的延迟寿命, 且随 着温度的降低而降低, 表现出典型的 TADF 效应. 这是 第一个同时具有 TADF 和 AIE 性质的草酸芳烃型分子, 虽然该工作未将材料运用于器件上, 但为后续科研工作 者开发大环类 AIDF 分子提供了可行的思路, 而且在超 分子化学和材料科学方面具有潜在的应用前景.

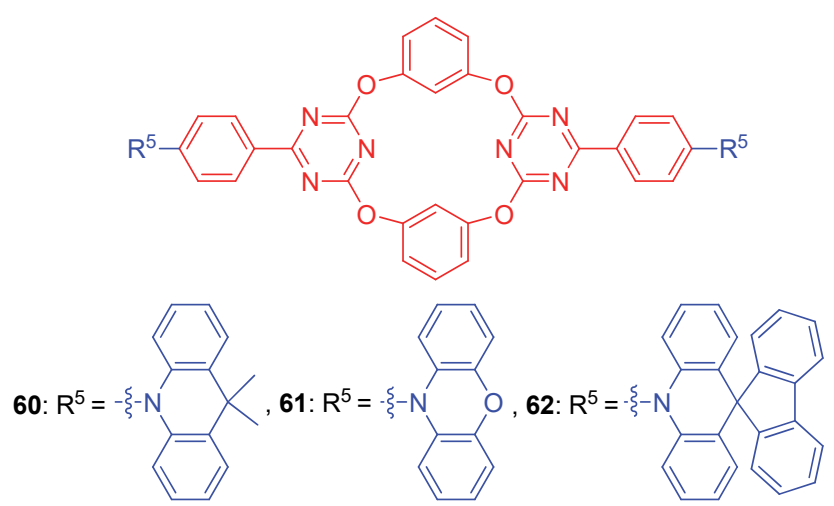

\section{4 喹喔啉及其衍生物}

上述各分子及器件已能涵盖较宽色域, 但基于 AIDF 分子设计的能高效发射红光的分子或器件依然不 多. 这是因为发光红移导致带隙减小, 非辐射跃迁几率 将增加，从而抑制辐射跃迁 ${ }^{[64-65]}$, 同时也因为红光分子 设计上 HOMO、LUMO 的分离, 导致电子跃迁几率降低, PLQY 降低. 喹喔啉因其具有 $\mathrm{sp}^{2}$ 杂化的 $\mathrm{N}$ 原子, 整体拥 有较强的吸电子能力, 且具有良好的平面性, 有六个可 
供修饰的位点, 因而被用作受体核心. 喹喔啉类分子往 往设计成具有大平面共轭和刚性的结构, 更长的共轭有 利于实现红光发射, 刚性结构有利于抑制非辐射跃迁, 所以该类分子多被利用于调控以发射红光或橙红光 (Scheme 4). 相关性质见表 4.

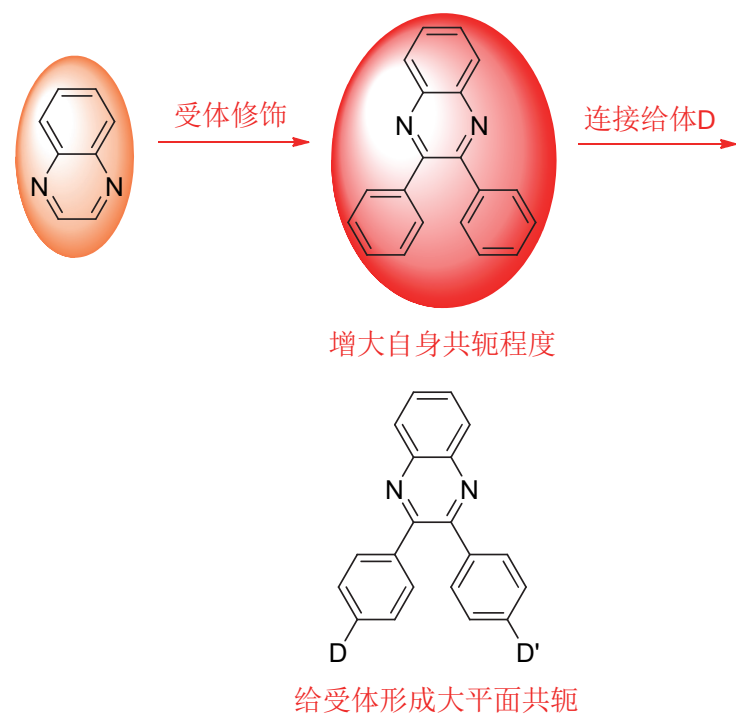

图式 4 喹喔啉类 AIDF 分子设计策略

Scheme 4 Molecular design strategy of quinoxaline-based AIDF compounds

2018 年, 杨楚罗课题组 ${ }^{[66]}$ 将 DMAC 和 PXZ 作给体 连接上喹喔啉受体, 设计了四个分子(分子 $63 \sim 66$ ), 由 于给体具有大的空间位阻, HOMO 和 LUMO 被有效分 离, 获得的 $\Delta E_{\mathrm{ST}}$ 分别为 $0.06 、 0.06 、 0.07 、 0.03 \mathrm{eV}$. 在

纯膜中四个分子发射波长分别为 541、551、594 和 618 $\mathrm{nm}$. 掺杂膜中, 以 PXZ 为供体的分子延迟寿命要比 DMAC 的短得多(分子 $65 、 66$ 为 $2.4 、 1.9 \mu \mathrm{s}$, 分子 63 、 64 为 $8.3 、 6.5 \mu \mathrm{s})$. 在掺杂膜中四个分子的 PLQY 分别

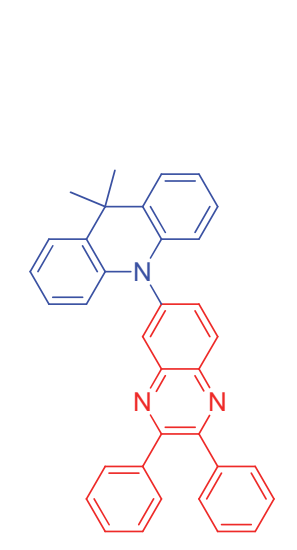

63

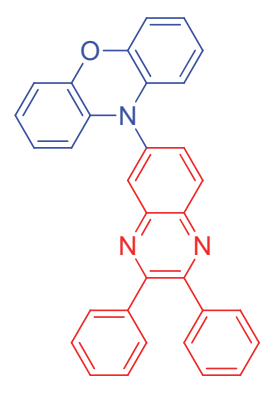

65
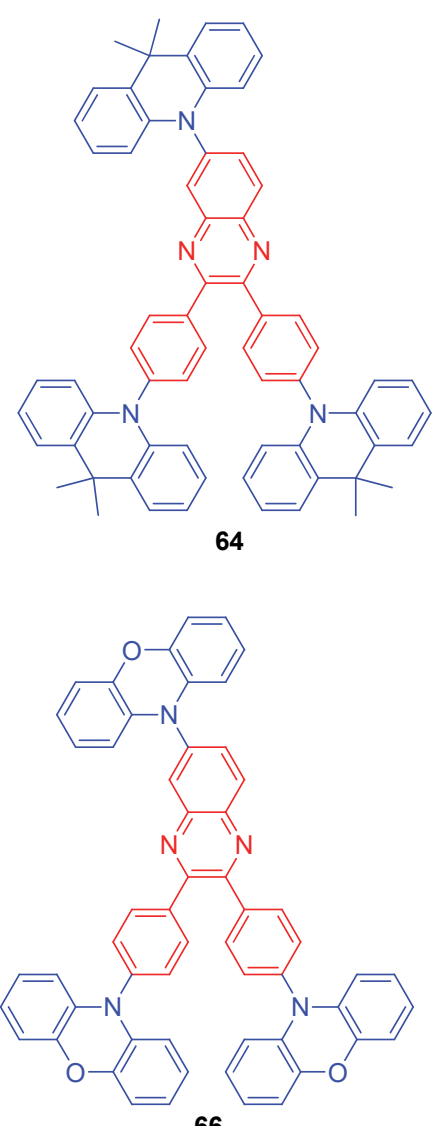

表 4 喹喔啉类 AIDF 分子在溶液中或分散在主体薄膜或非掺杂成膜中光致发光波长 $(\lambda \mathrm{PL})$ 、苂光量子产率(PLQY), 纯膜中苂光寿 命 $(\tau \mathrm{PF}, \tau \mathrm{DF})$ 、单重态-三重态能级差 $(\Delta E \mathrm{ST})$, 非掺杂或掺杂器件的电致发光波长 $(\lambda \mathrm{EL})$ 、最大外量子效率 $(\mathrm{EQE})$ 以及 $\mathrm{CIE}$ 色坐标

Table 4 PL peak $\left(\lambda_{\mathrm{PL}}\right)$ in solution or dispersed in a host film or in neat film, photoluminescence quantum yield (PLQY), lifetime in neat film $\left(\tau_{\mathrm{PF}}, \tau_{\mathrm{DF}}\right)$, singlet-triplet energy gap $\left(\Delta E_{\mathrm{ST}}\right)$ of quinoxaline-AIDF, EL peak $\left(\lambda_{\mathrm{EL}}\right)$ and maximum EQE, CIE for nondoped or doped devices, respecyively

\begin{tabular}{|c|c|c|c|c|c|c|c|c|c|}
\hline Compd. & $\lambda_{\mathrm{PL}} / \mathrm{nm}$ & PLQY/\% & $\tau \mathrm{PF} / \mathrm{ns}$ & $\tau_{\mathrm{DF}} / \mu \mathrm{s}$ & $\Delta E_{\mathrm{ST}} / \mathrm{eV}$ & $\lambda \mathrm{EL} / \mathrm{nm}$ & $\mathrm{EQE} / \%$ & CIE & Ref. \\
\hline 63 & $-/ 541$ & $-/ 74$ & 23 & 25.7 & 0.06 & $544 / 532$ & $10.1 / 13$ & $(0.39,0.58) /(0.34,0.60)$ & [66] \\
\hline 64 & $-/ 551$ & $-/ 84$ & 28 & 33 & 0.06 & $548 / 536$ & $12 / 22.4$ & $(0.40,0.57) /(0.35,0.59)$ & [66] \\
\hline 65 & $-/ 594$ & $-/ 73$ & 20 & 2.86 & 0.07 & $608 / 572$ & $5.6 / 11.1$ & $(0.56,0.43) /(0.49,0.50)$ & [66] \\
\hline 66 & $-/ 618$ & $-/ 76$ & 8.7 & 1.38 & 0.03 & $616 / 572$ & $3.3 / 14.1$ & $(0.60,0.40) /(0.50,0.49)$ & [66] \\
\hline 67 & $546 / 556$ & $13.8 / 43.4$ & 57 & 3.4 & 0.04 & $584 / 548$ & $10.1 / 23.5$ & - & [67] \\
\hline 68 & $551 / 561$ & $11.2 / 33.2$ & 68 & 3.3 & 0.04 & $588 / 548$ & $9.8 / 16.8$ & - & [67] \\
\hline 69 & $584 / 567$ & $-/ 19$ & 4.3 & 1340 & 0.1 & $544 / 544$ & $0.77 / 10.53$ & $(0.43,0.54) /(0.37,0.57)$ & [68] \\
\hline 70 & $601 / 563$ & $-/ 17$ & 4.9 & 1422 & 0.06 & $548 / 535$ & $0.64 / 4.95$ & $(0.40,0.54) /(0.34,0.56)$ & [68] \\
\hline 71 & $572 / 576$ & $10.5 / 76$ & 26.2 & 2.3 & 0.16 & $626 / 604$ & $5.6 / 15.4$ & $(0.61,0.38) /(0.56,0.44)$ & [69] \\
\hline 72 & $601 / 593$ & $8.3 / 62$ & 36.6 & 1.51 & 0.1 & $641 / 611$ & $2.9 / 9.2$ & $(0.64,0.35) /(0.58,0.41)$ & [69] \\
\hline 73 & $594 /-$ & - & 26 & 5.8 & 0.04 & - & - & - & [70] \\
\hline 74 & $604 /-$ & - & 26 & 2 & 0.03 & - & - & - & [70] \\
\hline 75 & $-/ 573$ & 一 & - & 一 & 0.02 & $567 / 554$ & $7.2 / 5$ & - & [71] \\
\hline 76 & - & - & - & - & 0.04 & - & - & - & [71] \\
\hline 77 & - & - & - & - & 0.05 & - & - & - & [71] \\
\hline 78 & - & - & - & - & 0.02 & - & - & - & [71] \\
\hline
\end{tabular}


为 $74 \% 、 84 \% 、 73 \% 、 76 \%$. 结构为 $\mathrm{ITO} / \mathrm{MoO}_{3}(10 \mathrm{~nm}) /$ TAPC $(50 \mathrm{~nm}) / \mathrm{mCP}(10 \mathrm{~nm}) / \mathrm{CBP}: \mathrm{TADF}$ EML $(10 \%, 20$ $\mathrm{nm}) / \mathrm{Bphen}(45 \mathrm{~nm}) / \mathrm{LiF}(1 \mathrm{~nm}) / \mathrm{Al}$ 的掺杂器件中, 64 获得 了最好的 EL 性能, 最大 CE、PE、 EQE 分别为 $80.3 \mathrm{~cd} \cdot$ $\mathrm{A}^{-1}, 64.1 \mathrm{~lm} \cdot \mathrm{W}^{-1}$ 和 $22.4 \% .66$ 显示出橙光发射, $E Q E$ 为 $14.1 \%$, 是当时性能较高的橙光 OLED. 非掺杂器件 ITO/ $\mathrm{MoO}_{3}(10 \mathrm{~nm}) / \mathrm{TAPC}(50 \mathrm{~nm}) / \mathrm{mCP}(10 \mathrm{~nm}) / \mathrm{EML}$ (20 $\mathrm{nm}) / \mathrm{Bphen}(45 \mathrm{~nm}) / \mathrm{LiF} / \mathrm{Al}, \mathbf{6 6}$ 获得最好的 EL 性能, 最大 $C E 、 P E 、 E Q E$ 分别为 $41.2 \mathrm{~cd} \bullet \mathrm{A}^{-1}, 45.4 \mathrm{~lm} \cdot \mathrm{W}^{-1}$ 和 $12.0 \%$. 随后, 该课题组 ${ }^{[67]}$ 通过将氟原子引入喹喔啉设计 了两个分子 67、68, 利用吸电子能力较弱的氟原子, 增 强其分子间电子偶联能力. 其 $\Delta E_{\mathrm{ST}}$ 都为 $0.04 \mathrm{eV}$, 在纯 膜中的延迟寿命分别为 3.4 和 $3.3 \mu \mathrm{s}$, 从溶液到纯膜, PLQY 分别从 $13.8 \%$ 提高到 $43.4 \%$, 从 $11.2 \%$ 提高到 $33.2 \%$, 掺杂膜中达 $99.6 \% 、 68.3 \%$. 掺杂器件结构为 ITO/ $\mathrm{MoO}_{3}(10 \mathrm{~nm}) / \mathrm{TAPC}: \mathrm{MoO}_{3}(20 \%, 50 \mathrm{~nm}) / \mathrm{TAPC}(20$ $\mathrm{nm}) / \mathrm{CBP}: \operatorname{TADF}(10 \%, 20 \mathrm{~nm}) / \mathrm{Bphen}(45 \mathrm{~nm}) / \mathrm{LiF}(1 \mathrm{~nm}) /$ $\mathrm{Al}$, 基于 67 的器件获得了最好的 EL 性能分别为 78.3 $\mathrm{cd} \bullet \mathrm{A}^{-1}, 91.1 \mathrm{~lm} \bullet \mathrm{W}^{-1}$ 和 $23.5 \%$ 的 $\mathrm{EQE}$ ，体现了氟原子取 代后对于增强掺杂器件发光效率的作用. 非掺杂器件结 构为 $\mathrm{ITO} / \mathrm{MoO}_{3}(10 \mathrm{~nm}) / \mathrm{TAPC}(50 \mathrm{~nm}) / \mathrm{mCP}(10 \mathrm{~nm}) /$ EML $(30 \mathrm{~nm}) / \mathrm{Bphen}(45 \mathrm{~nm}) / \mathrm{LiF}(1 \mathrm{~nm}) / \mathrm{Al}, \mathbf{6 7} 、 68$ 都显 示出橙色发光, $\mathrm{EQE}$ 分别为 $10.1 \%$ 和 $9.8 \%$.

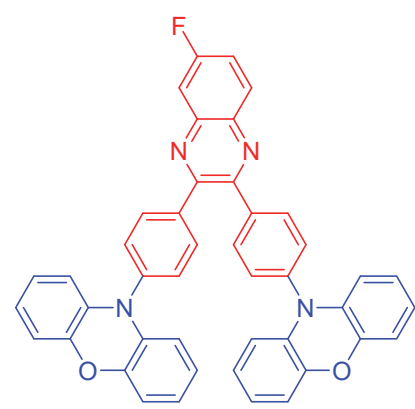

67

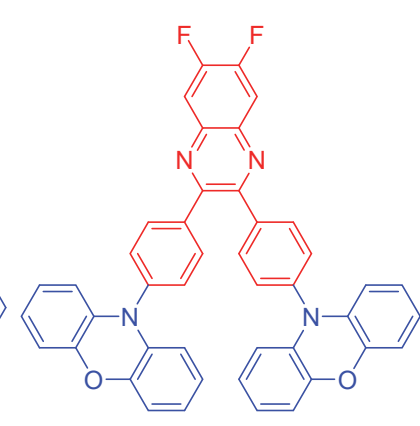

68
2020 年, Grazulevicius 等[68]通过将甲氧基、叔丁基 等连接到咔唑上调节给电子能力, 设计了两个不对称 D-A-D'型结构的新型多功能材料分子 69、70, 由于给体 的不同显示出不同的刺激响应变化及 AIE 效应. 含有未 修饰咔唑取代的 69 具有较强的偶极矩和分子间的相互 作用, 而 70 中叔丁基咔唑单元的结合产生了更有效的 热激活延迟苂光, 提高了热稳定性和更强的刺激响应变 化. 其 $\Delta E_{\mathrm{ST}}$ 分别为 0.10 和 $0.06 \mathrm{eV}$, 在掺杂膜中的 PLQY 分别为 $19 \%$ 和 $17 \%$. 掺杂器件结构为 $\mathrm{ITO} / \mathrm{MoO}_{3}(0.5$ $\mathrm{nm}) / \mathrm{NPB}(35 \mathrm{~nm}) / \mathrm{OTQx}$ or OCQx $(w=10 \%)$ :TCTA $(20$ $\mathrm{nm}) / \mathrm{TSPO} 1(7 \mathrm{~nm}) / \mathrm{TPBi}(30 \mathrm{~nm}) / \mathrm{LiF}(0.5 \mathrm{~nm}) / \mathrm{Al}, 69$ 获 得了最好的 EL 性能, 最大 CE、PE、 $\mathrm{EQE}$ 分别为 33.6 $\mathrm{cd} \cdot \mathrm{A}^{-1}, 21.1 \mathrm{~lm} \cdot \mathrm{W}^{-1}$ 和 $10.5 \%$.

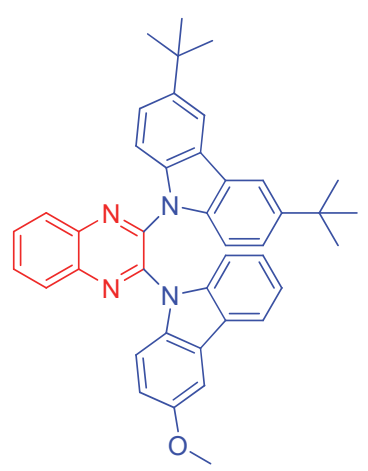

69

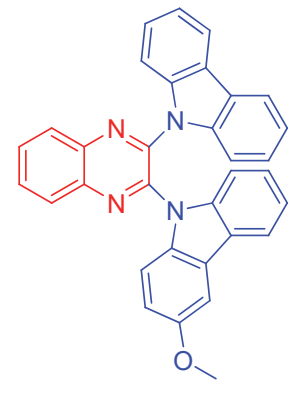

70
在上述分子的基础上，王利祥课题组 ${ }^{[69]}$ 稠合了喹 喔啉下方两个游离的苯环, 合成新的喹喔啉衍生物受体 (DBPZ). 利用 TAT 和 DBPZ分别作供体和受体设计了分 子 71、72. 大的空间位阻产生了小的 $\Delta E_{\mathrm{ST}}$, 分别为 0.16 和 $0.10 \mathrm{eV}$. 由于两个基团的刚性和大平面共轭结构, 两 个分子拥有高的 PLQY, 分别为 $76 \%$ 和 $62 \%$. 由于咔唑 杂环上烷基链的引入, 两个分子具有良好的溶解度, 用 溶液加工的方法制备结构为 ITO/PEDOT:PSS $(40 \mathrm{~nm}) /$ EML $(50 \mathrm{~nm}) / \operatorname{TmPyPB}(42 \mathrm{~nm}) / \mathrm{LiF}(1 \mathrm{~nm}) / \mathrm{Al}(100 \mathrm{~nm})$ 的 掺杂器件. 基于 71 的器件发射出 $604 \mathrm{~nm}$ 的红光, 最大 $C E 、 P E 、 E Q E$ 分别为 $29.7 \mathrm{~cd} \cdot \mathrm{A}^{-1}, 23.3 \mathrm{~lm} \cdot \mathrm{W}^{-1}$ 和 $15.4 \%$. 基于 71、72 溶液加工的非掺杂红光 OLED 的 EQE 分别 为 $5.6 \%$ 和 $2.9 \%$.
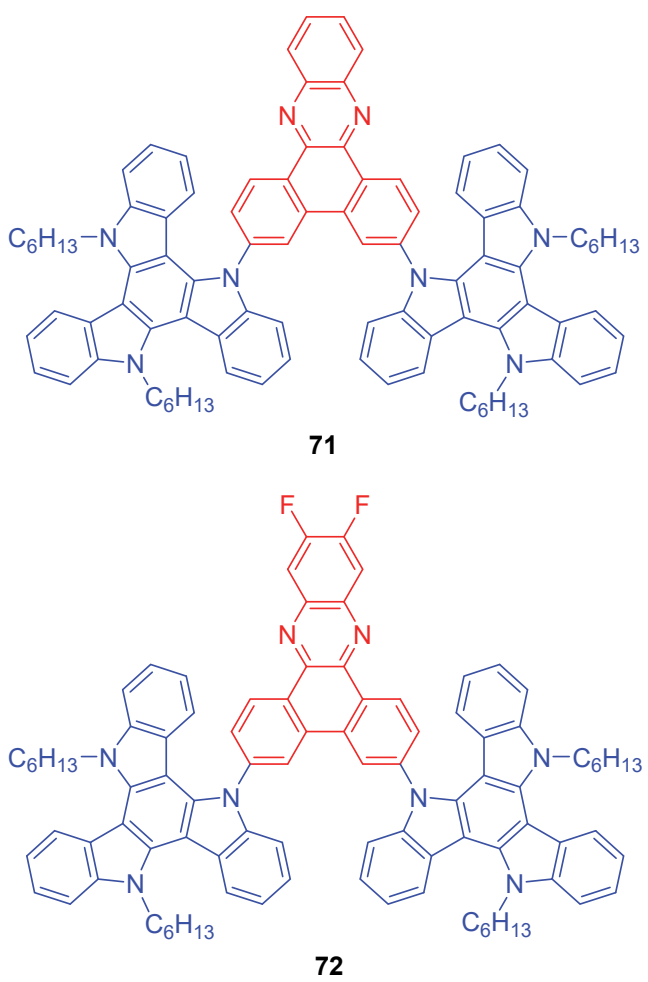
同样基于二苯基-喹喔啉受体，杨楚罗课题组 ${ }^{[70]}$ 通 过将两个氧基引入喹喔啉, 并且将其与苯或氰基苯相连 作为受体, 增大受体的吸电子能力, 以 DMAC 作为供体 设计了两个分子 73、74, 其 $\Delta E_{\mathrm{ST}}$ 分别为 0.04 和 $0.03 \mathrm{eV}$. 溶液中表现出 AIE 和 TADF 性质, 粉末态时呈现多色 MCL 和 TADF 特征, 晶态时表现出同质多晶依赖的 TADF 特征. 两个分子在粉末态时表现出多色力致变色 性质. 其初始状态下的黄绿色(或橙色)固体, 经研磨后 表现出红橙光发射, 再经二氯甲烷熏蒸后又恢复至黄色 发光, 且经不同处理的固体均表现出典型的 TADF 发射. 同质多晶现象为进一步探究聚集态和 TADF 性质提供了 独特的视角. 每种聚集态都表现出不同的 TADF 发射波 长、寿命和发光效率. 单晶衍射数据显示二者结构具有 多重易变构象, 且含有多种形式和作用强度的分子间作 用力. DMAC 更加扭曲的构象具有更高的激发态, 常出 现在黄色单晶结构中; 分子间存在较多而且较强的 $\pi-\pi$ 作用则会导致光谱的红移和发光效率的降低. 从分子水 平到聚集态: 分子结构决定了 TADF 的特性, 聚集态中 不同的构象和分子间作用方式进一步影响了发射波长、 寿命和发光效率.
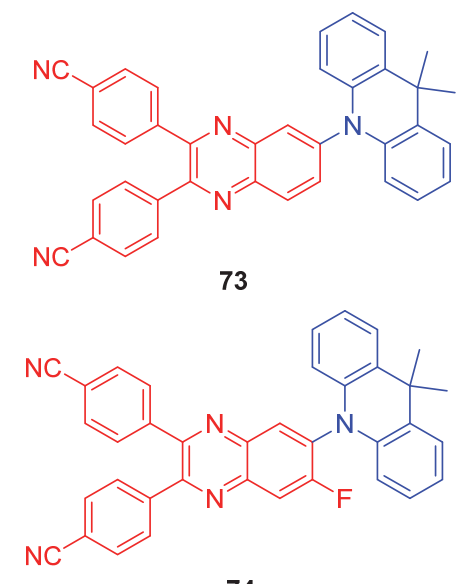

74

随后, 该课题组 ${ }^{[71]}$ 将二苯基-喹喔啉受体中苯环替 换为吡啶环设计合成 DPQ、DPP 两个不同受体, 利用 DMAC、PXZ 作给体设计了四个 AIDF 分子 75 78. 新 受体中额外的氮原子可以提供多个分子间/分子内氢键, 实现多色刺激响应 $\mathrm{MCL}$ 现象. 其 $\Delta E_{\mathrm{ST}}$ 分别为 0.02 、 $0.04 、 0.05 、 0.02 \mathrm{eV}$. 基于 75 制备了两个器件, 结构为 ITO/HAT-CN (5 nm)/TAPC(30 nm)/mCP(10 nm)/DPEPO: $75(w=10 \%)$ EML $(20 \mathrm{~nm}) / \mathrm{DPEPO}(10 \mathrm{~nm}) / \mathrm{TmPyPB}(30$ $\mathrm{nm}) / \mathrm{Liq}(1.5 \mathrm{~nm}) / \mathrm{Al}$ 的掺杂器件, 最大 $\mathrm{CE} 、 \mathrm{PE} 、 \mathrm{EQE}$ 分 别为 $35.3 \mathrm{~cd} \bullet \mathrm{A}^{-1}, 31.8 \mathrm{~lm} \cdot \mathrm{W}^{-1}$ 和 $11.3 \%$. 结构为 ITO/ HAT-CN (15 nm)/TAPC (60 nm)/TCTA $(10 \mathrm{~nm}) / 75$ (20 $\mathrm{nm}) / \mathrm{BPhen}(45 \mathrm{~nm}) / \mathrm{LiF}(1 \mathrm{~nm}) / \mathrm{Al}$ 的非掺杂器件, 最大 $\mathrm{CE} 、 \mathrm{PE} 、 \mathrm{EQE}$ 分别为 $20.5 \mathrm{~cd} \bullet \mathrm{A}^{-1}, 20.2 \mathrm{~lm} \bullet \mathrm{W}^{-1}$ 和 $7.2 \%$.

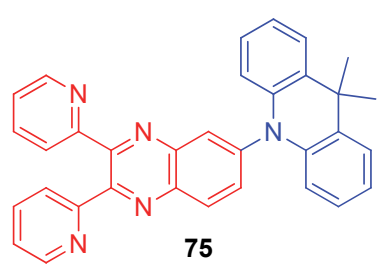<smiles>CC1(C)c2ccccc2N(c2cnc3nc(-c4ccccn4)c(-c4ccccn4)nc3c2)c2ccccc21</smiles><smiles>c1ccc(-c2nc3ccc(N4c5ccccc5Oc5ccccc54)cc3nc2-c2ccccn2)nc1</smiles><smiles>c1ccc(-c2nc3cc(N4c5ccccc5Oc5ccccc54)cnc3nc2-c2ccccn2)nc1</smiles>

\section{5 其他受体}

除了以上四类常见的受体外，近年来，一些新颖的 受体相继被开发，在 AIDF 分子的设计上自成体系. 其 中, 比较典型的受体分子有基于蒽醌的衍生物、基于萘 啶的衍生物、邻碳硼烷等. 相关性质见表 5 .

早在 2017 年, 蒋伟课题组 ${ }^{[72]}$ 便利用咔唑和蒽醌设 计了分子 79, 同时具有 ML、TADF 和 AIE. 其在不同的 溶液中形成不同的晶体 Y、R. Y 晶体显示出 $541 \mathrm{~nm}$ 的 黄光发射, $\mathrm{R}$ 晶体显示出 $604 \mathrm{~nm}$ 的红光发射, 延迟苂光 寿命分别为 1.76 和 $1.92 \mu \mathrm{s}$. 溶液加工制备非掺杂器件, ITO/PEDOT:PSS (40 nm)/Cz-AQ (40 nm)/TPBI (30 nm)/ $\mathrm{Cs}_{2} \mathrm{CO}_{3}(2 \mathrm{~nm}) / \mathrm{Al}(120 \mathrm{~nm})$, 用不同的溶液沉积时显示 出不同发射峰, $\mathrm{Y} 、 \mathrm{R}$ 晶体的电致发光波长分别为 600 和 $680 \mathrm{~nm}$.

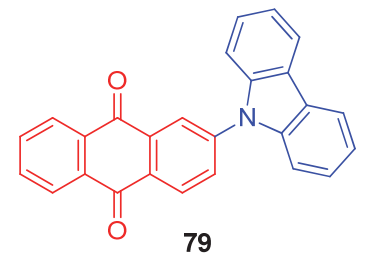

Grazulevicius 课题组 ${ }^{[73]}$ 使用全氟联苯作为受体设计 了 4 个分子 $80 \sim 83$. 其 $\Delta E_{\mathrm{ST}}$ 分别为 $0.16,0.06,0.03,0.06$ eV. 四个分子都显示出蓝光发射, 从溶液到薄膜 81 的 PLQY 从 3\%升到 $61 \%$. 四个分子都表现出蓝色或天蓝 色发光. 基于 83 制备了结构为 $\mathrm{ITO} / \mathrm{MoO}_{3} / \mathrm{NPB} / \mathbf{8 3}$ : $\mathrm{TCz} 1 / \mathrm{TPBi} / \mathrm{Ca} / \mathrm{Al}$ 的掺杂器件, $\mathrm{EL}$ 性能为 $30.8 \mathrm{~cd} \bullet \mathrm{A}^{-1}$, $22.7 \mathrm{~lm} \cdot \mathrm{W}^{-1}$ 和 $16.3 \%$ 的 $\mathrm{EQE}$, 由其制备的同样结构的非 掺杂器件 $\mathrm{EQE}$ 为 $6.6 \%$.

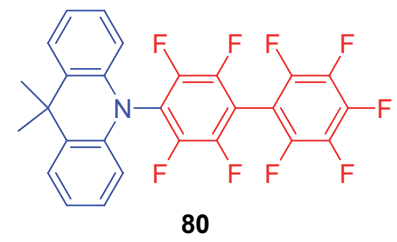


表 5 其他 $\mathrm{AIDF}$ 分子在溶液中或分散在主体薄膜或非掺杂成膜中光致发光波长 $\left(\lambda_{\mathrm{PL}}\right)$ 、苂光量子产率 $(\mathrm{PLQY})$, 纯膜中苂光寿命 $(\tau \mathrm{PF}$, $\left.\tau_{\mathrm{DF}}\right)$ 、单重态-三重态能级差 $\left(\Delta E_{\mathrm{ST}}\right)$, 非掺杂或掺杂器件电致发光波长 $\left(\lambda_{\mathrm{EL}}\right)$ 、最大外量子效率 $(\mathrm{EQE})$ 以及 CIE 色坐标

Table 5 PL peak $\left(\lambda_{\mathrm{PL}}\right)$ in solution or dispersed in a host film or in neat film, photoluminescence quantum yield (PLQY), lifetime in neat film $\left(\tau_{\mathrm{PF}}, \tau_{\mathrm{DF}}\right)$, singlet-triplet energy gap $\left(\Delta E_{\mathrm{ST}}\right)$, EL peak $\left(\lambda_{\mathrm{EL}}\right)$ of other AIDF, maximum EQE, CIE for based nondoped or doped devices, respectively

\begin{tabular}{|c|c|c|c|c|c|c|c|c|c|}
\hline Compd. & $\lambda_{\mathrm{PL}} / \mathrm{nm}$ & PLQY/\% & $\tau_{\mathrm{PF}} / \mathrm{ns}$ & $\tau_{\mathrm{DF}} / \mu \mathrm{s}$ & $\Delta E_{\mathrm{ST}} / \mathrm{eV}$ & $\lambda_{\mathrm{EL}} / \mathrm{nm}$ & EQE/\% & CIE & Ref. \\
\hline 79 & $-/ 541$ & $0.35 / 0.59$ & 77.3 & 1.71 & - & $600 /-$ & $1.15 /-$ & $(0.50,0.42) /-$ & [68] \\
\hline 79 & $-/ 604$ & $0.12 / 0.28$ & 81.6 & 1.92 & - & $680 /-$ & $0.75 /-$ & $(0.68,0.30) /-$ & [68] \\
\hline 80 & $493 / 418$ & $-/ 27$ & - & - & 0.16 & $-/ 480$ & $-/ 3.3$ & $-/(0.178,0.27)$ & [69] \\
\hline 81 & $497 / 482$ & $-/ 34$ & - & - & 0.06 & $-/ 487$ & $-/ 3.9$ & $-/(0.20,0.29)$ & [69] \\
\hline 82 & $528 / 463$ & $3 / 61$ & - & - & 0.03 & $499 /-$ & $6.6 /-$ & $(0.22,0.39) /-$ & [69] \\
\hline 83 & $516 / 494$ & $8 / 46$ & - & - & 0.06 & -1499 & $-/ 16.3$ & $-/(0.20,0.42)$ & [69] \\
\hline 84 & $495 / 520$ & $-/ 38.7$ & 22.3 & 1.67 & 0.15 & $537 / 496$ & $3.5 / 9.3$ & - & [70] \\
\hline 85 & $530 / 538$ & $-/ 19.6$ & 12.6 & 1.43 & 0.11 & $563 / 527$ & $2.3 / 6.3$ & - & [70] \\
\hline 86 & $531 / 553$ & $-/ 22.1$ & 11.3 & 1.33 & 0.02 & $550 / 547$ & $2.9 / 3.5$ & - & [70] \\
\hline 87 & $585 / 580$ & $-/ 5.3$ & 12.6 & 0.46 & 0.11 & $597 / 571$ & $0.6 / 1.7$ & - & [70] \\
\hline 88 & $-/ 516$ & $-/ 40.82$ & - & 1.37 & 0.06 & $514 / 488$ & $11 / 14.1$ & $(0.28,0.52) /(0.19,0.41)$ & [71] \\
\hline 89 & $-/ 539$ & $-/ 36.05$ & - & 1.26 & 0.07 & $534 / 528$ & $9.7 / 13$ & $(0.36,0.55) /(0.30,0.50)$ & [71] \\
\hline 90 & $-/ 566$ & $-/ 13.4$ & - & 0.97 & 0.09 & $568 / 544$ & $3.7 / 13.4$ & $(0.48,0.48) /(0.40,0.55)$ & [71] \\
\hline 91 & $556 / 551$ & $-/ 55$ & 40 & 2.98 & 0.03 & $558 / 542$ & $12 / 16.8$ & $(0.43,0.54) /(0.38,0.57)$ & [72] \\
\hline 92 & $643 / 632$ & -15 & 26.8 & 0.77 & 0.02 & $-/ 588$ & $-/ 8.4$ & $-/(0.47,0.49)$ & [72] \\
\hline 93 & $588 / 593$ & $-/ 26$ & - & - & 0.15 & $628 / 602$ & $1.53 / 7.21$ & $(0.65,0.34) /(0.59,0.41)$ & [73] \\
\hline 94 & $592 / 555$ & $-/ 55$ & - & - & 0.12 & $583 / 538$ & $1.39 / 7.59$ & $(0.53,0.46) /(0.35,0.60)$ & [73] \\
\hline 95 & $-/ 557$ & - & - & - & 0.006 & - & - & - & [74] \\
\hline 96 & - & - & - & - & 0.012 & - & - & - & [74] \\
\hline 97 & $-/ 551$ & - & - & - & 0.009 & - & - & - & [74] \\
\hline 98 & $-/ 626$ & - & - & - & 0.023 & - & - & - & [74] \\
\hline 99 & $-/ 727$ & - & - & - & 0.052 & - & - & - & [74] \\
\hline 100 & $-/ 946$ & - & - & - & 0.09 & - & - & - & [74] \\
\hline 101 & $-/ 469$ & $46 / 74$ & 0.95 & 2.3 & 0.08 & $473 / 478$ & $20.9 / 27.6$ & $(0.14,0.20) /(0.14,0.26)$ & [75] \\
\hline 102 & $-/ 473$ & $27 / 11$ & 1.3 & 1.7 & 0.06 & $-/ 484$ & $-/ 23.9$ & $-/(0.14,0.32)$ & [75] \\
\hline 103 & $-/ 464$ & $42 / 83$ & 0.95 & 3.2 & 0.11 & $473 / 476$ & $17.4 / 15.7$ & $(0.14,0.20) /(0.14,0.22)$ & [75] \\
\hline 104 & $-/ 465$ & $22 / 23$ & 0.54 & 1.8 & 0.11 & -1478 & $-/ 21.6$ & $-/(0.14,0.26)$ & [75] \\
\hline 105 & $-/ 444$ & $-/ 32$ & - & - & 0.09 & $449 /-$ & $3.5 /-$ & $(0.17,0.13) /-$ & [76] \\
\hline 106 & $-/ 453$ & $-/ 37$ & - & - & 0.09 & $451 /-$ & $3.8 /-$ & $(0.18,0.17) /-$ & [76] \\
\hline 107 & $-/ 448$ & $-/ 44$ & - & - & 0.06 & $453 /-$ & $5.2 /-$ & $(0.17,0.16) /-$ & [76] \\
\hline 108 & $-/ 455$ & $-/ 48$ & - & - & 0.07 & $455 /-$ & $6.1 /-$ & $(0.18,0.20) /-$ & [76] \\
\hline 109 & $-/ 471$ & $-/ 65$ & - & - & 0.01 & $471 /-$ & $14.4 /-$ & $(0.15,0.21) /-$ & [76] \\
\hline 110 & $-/ 480$ & $-/ 70$ & - & - & 0.02 & $474 /-$ & $15 /-$ & $(0.16,0.27) /-$ & [76] \\
\hline 111 & $473 / 502$ & $-/ 38.7$ & 38 & 3.1 & 0.16 & $508 /-$ & $7.3 /-$ & $(0.28,0.54) /-$ & [77] \\
\hline 112 & $476 / 504$ & $-/ 45.7$ & 56 & 3.8 & 0.15 & $508 /-$ & $13.9 /-$ & $(0.27,0.54) /-$ & [77] \\
\hline 113 & $480 / 504$ & $-/ 69.6$ & 77 & 4.5 & 0.13 & $508 /-$ & $20.1 /-$ & $(0.27,0.53) /-$ & [77] \\
\hline 114 & $531 / 572$ & $-/ 35$ & 22.8 & 0.85 & 0.09 & $574 /-$ & $5.6 /-$ & $(0.48,0.54) /-$ & [78] \\
\hline 115 & $489 / 513$ & $-/ 86$ & 10.7 & 0.32 & 0.01 & $536 / 496$ & $14.5 / 16.2$ & $(0.34,0.56) /(0.22,0.39)$ & [78] \\
\hline
\end{tabular}

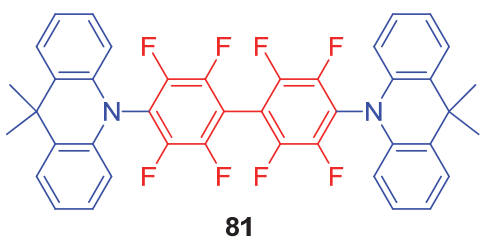

唐本忠课题组 ${ }^{[74]}$ 开发了系列可用于圆偏振有机发 光二极管(CPOLED)的含双菜发光对映体分子 $84 \sim 87$. 在纯膜中，其 PLQY 分别为 $38.7 \% 、 19.6 \% 、 22.1 \% 、 5.3 \%$, 延迟寿命为 $1.67 、 1.43 、 1.33 、 0.46 \mu$ s. 基于 84 制备结

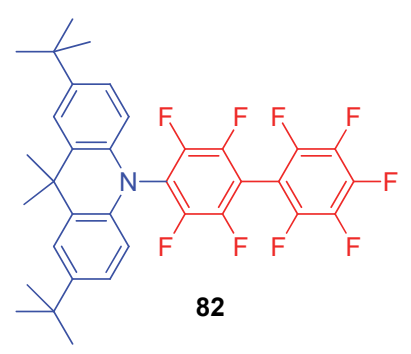

构为 ITO/HATCN $(10 \mathrm{~nm}) /$ TAPC:HATCN $(5: 2 w / w, 60$ $\mathrm{nm}) / \mathrm{TCTA}(20 \mathrm{~nm}) / \mathrm{mCP}: 10 \% \mathrm{~S}-\mathrm{BN}-\mathrm{CF}(20 \mathrm{~nm}) / \mathrm{BmPy}-$ 


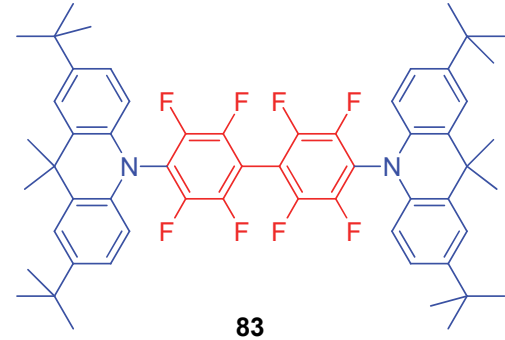

83

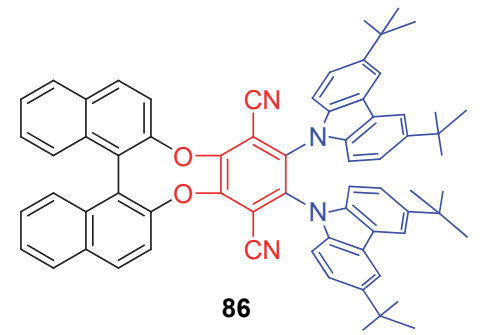

86

$\mathrm{PB}(10 \mathrm{~nm}) / \mathrm{BmPyPB}: 8 \%$ Liq $(40 \mathrm{~nm}) / \mathrm{Liq}(1.5 \mathrm{~nm}) / \mathrm{Al}$ 的 掺杂器件, 最大 $\mathrm{CE} 、 \mathrm{PE} 、 \mathrm{EQE}$ 分别为 $24.6 \mathrm{~cd} \cdot \mathrm{A}^{-1}, 19.6$ $\mathrm{lm} \cdot \mathrm{W}^{-1}$ 和 $9.3 \%$. 基于其制备的非掺杂器件获得了 $3.5 \%$ 的 EQE.

2019 年, 陈传峰课题组 ${ }^{[75]}$ 利用 1,8 -菜啶作受体设计 三个分子 88、89、90. 其 $\Delta E_{\mathrm{ST}}$ 分别为 $0.06 、 0.07 、 0.09$ $\mathrm{eV}$, 纯膜中, 其 PLQY 分别为 $40.8 \% 、 36.1 \%$ 和 $13.4 \%$, 延 迟寿命为 $1.37 、 1.26 、 0.97 \mu \mathrm{s}$. 器件结构为 ITO/TAPC (30 $\mathrm{nm}) / \mathrm{TCTA}(5 \mathrm{~nm}) / \mathrm{EML}(20 \mathrm{~nm}) / \mathrm{Tm}_{3} \mathrm{PyPB}$ (60 nm)/LiF (1 $\mathrm{nm}) / \mathrm{Al}(100 \mathrm{~nm})$, 在掺杂于 $\mathrm{mCP}$ 主体材料的器件中, 三 个分子都有较高的 $\mathrm{EQE}$ ，分别为 $14.1 \% 、 13.0 \% 、 13.4 \%$. 在非掺杂器件中, 基于 $\mathbf{8 8}$ 的器件获得了最好的 EL 性能, 分别为 $34.7 \mathrm{~cd} \bullet \mathrm{A}^{-1}, 32.0 \mathrm{~lm} \cdot \mathrm{W}^{-1}$ 和 $11.0 \%$ 的 $\mathrm{EQE}$.
88

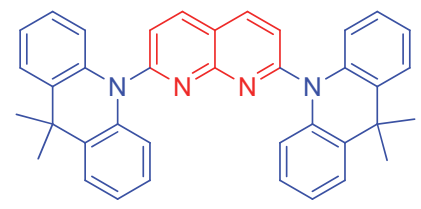

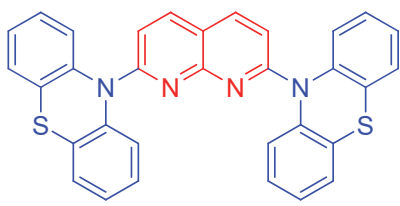

89<smiles>c1ccc2c(c1)Oc1ccccc1N2c1ccc2ccc(N3c4ccccc4Oc4ccccc43)nc2n1</smiles>

90

同样是用荎啶作受体, 杨楚罗课题组 ${ }^{[76]}$ 设计了分 子91、92. 两个分子拥有近乎正交的分子构型, 92 由于 在受体中引入氰基, HOMO 与 LUMO 都更低, 同时给受 体间的二面角也更大, 经密度泛函理论(DFT)计算得到 其 $\Delta E_{\mathrm{ST}}$ 分别为 0.03 和 $0.02 \mathrm{eV}$. 在纯膜中, 91 和 92 的 PLQY 分别为 $55 \%$ 和 $5 \%$, 延迟寿命为 2.98 和 $0.77 \mu \mathrm{s}$, 发 光波长为 551 和 $632 \mathrm{~nm}$. 92 更低的 PLQY 归因于氰基对

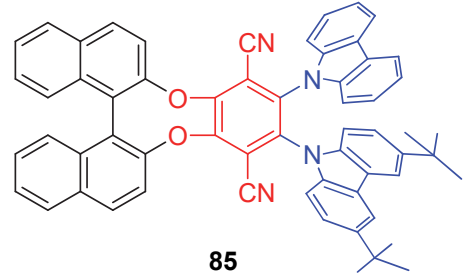

84<smiles>CC1(C)c2ccccc2N(c2c(F)c(C#N)c(Oc3ccc4ccccc4c3-c3c(Oc4ccc5ccccc5c4C#N)ccc4ccccc34)c(C#N)c2Br)c2ccccc21</smiles>

激子的猝灭效应以及长波长发射导致的非辐射跃迁速 率加快. 掺杂器件结构为 ITO/TAPC (30 nm)/TCTA (5 $\mathrm{nm}) / \mathrm{EML}(15 \mathrm{~nm}) / \mathrm{TmPyPB}(65 \mathrm{~nm}) / \mathrm{LiF}(1 \mathrm{~nm}) / \mathrm{Al}(100$ $\mathrm{nm}), 91$ 获得了最好的 EL 性能, 最大 CE、PE、EQE 分 别为 $58.1 \mathrm{~cd} \cdot \mathrm{A}^{-1}, 50.7 \mathrm{~lm} \cdot \mathrm{W}^{-1}$ 和 $16.8 \%$, 在同样结构的 非掺杂器件中, 最大 $\mathrm{CE} 、 \mathrm{PE} 、 \mathrm{EQE}$ 分别为 $38.5 \mathrm{~cd} \bullet \mathrm{A}^{-1}$, $30.2 \mathrm{~lm} \cdot \mathrm{W}^{-1}$ 和 $12.0 \%$

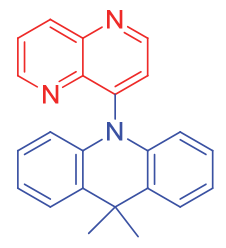

91

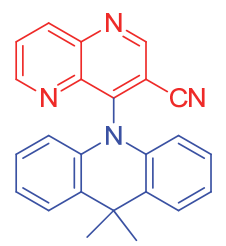

92
随后, 彭争春课题组 ${ }^{[77]}$ 利用中央萘二甲酰亚胺作 受体，芳胺作给体设计了两个发射橙红光的分子 93、94. 其 $\Delta E_{\mathrm{ST}}$ 分别为 $0.15 、 0.12 \mathrm{eV}$ ，纯膜的 PLQY 分别为 $26 \%$ 、 $55 \%$, 掺杂膜中 PLQY 则为 $62 \% 、 87 \%$. 两个分子具有 大的空间位阻，聚集态下 $\pi-\pi$ 相互作用和分子间作用被 抑制, 从而产生 AIE. 器件结构为 ITO/HAT-CN (15 nm)/ TAPC (40 nm)/TCTA (5 nm)/EML (20 nm)/TmPyPB (40 $\mathrm{nm}) / \mathrm{LiF}(1 \mathrm{~nm}) / \mathrm{Al}(100 \mathrm{~nm})$. 在非掺杂器件中 93 表现出 红光发射, 波长为 $628 \mathrm{~nm}$, 色坐标为 $(0.64,0.35), \mathrm{EL}$ 性 能为 $2.44 \mathrm{~cd} \bullet \mathrm{A}^{-1}, 1.85 \mathrm{~lm} \cdot \mathrm{W}^{-1}$. 基于 94 的掺杂器件获得 最好的 $\mathrm{EL}$ 性能为 $27.95 \mathrm{~cd} \cdot \mathrm{A}^{-1}, 20.97 \mathrm{~lm} \cdot \mathrm{W}^{-1}$ 和 $7.59 \%$ 的 $\mathrm{EQE}$ ，但发射波长为 $538 \mathrm{~nm}$.

苏忠民课题组 ${ }^{[78]}$ 合成并系统研究了系列邻碳硓烷 衍生物 95 100. 分子 95 和 96 的 HOMO、LUMO 几乎 没有重叠，其余四个分子重叠也极小，因此这六个分子 $\Delta E_{\mathrm{ST}}$ 都很小, 在 $0.006 \sim 0.09 \mathrm{eV}$ 之间. 作者研究了邻碳 硼烷对 AIE 性质的影响, 指出邻碳嗍烷上给受体可以 

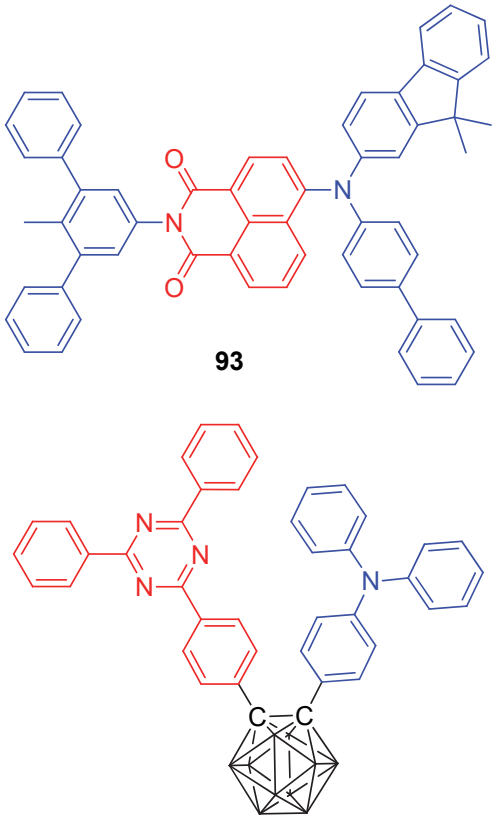

96
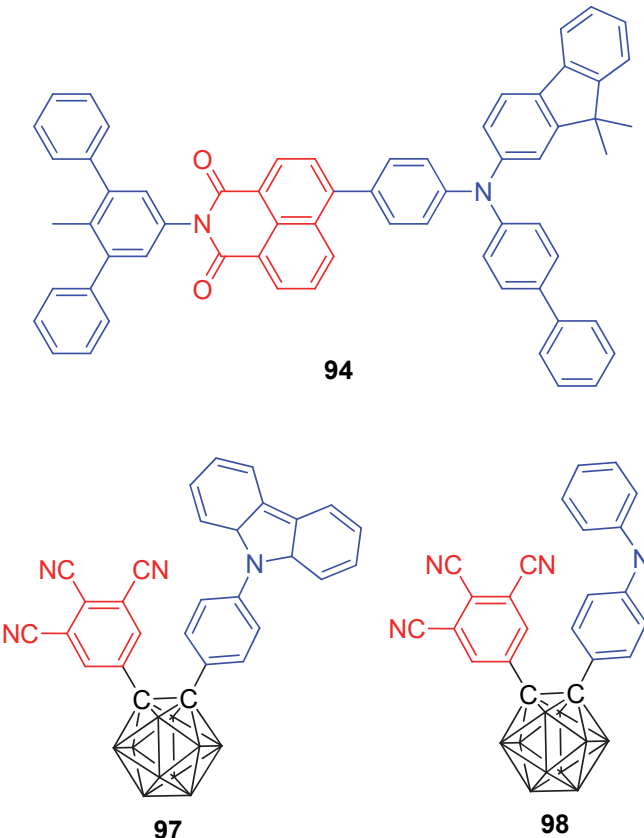

97
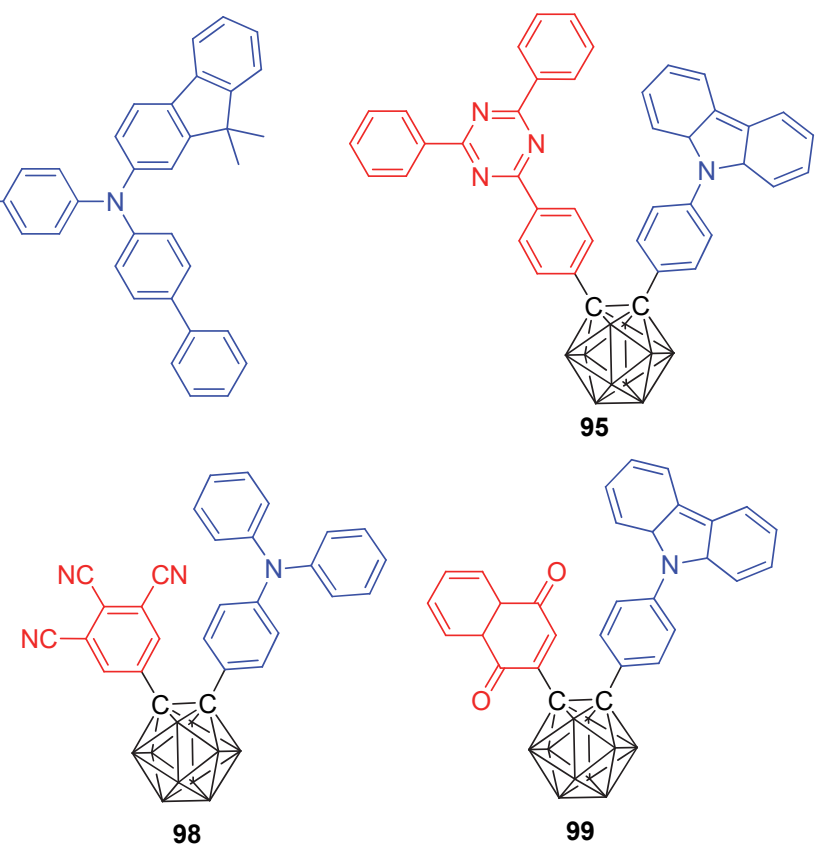

自由旋转, 因此分子在溶液中的 $\mathrm{CT}$ 过程被减弱, 聚集 后则增强, 这对其产生 AIE 有利. 作者指出聚集态下的 分子, $\mathrm{S}_{1}$ 到 $\mathrm{S}_{0}$ 的结构弛豫显著降低, 减少了能量损失和 非辐射跃迁, 从而产生 AIE. 六个分子都具有 TADF 和 AIE 性质, 但由于分子 97 100 在水中观测不到 AIE, 作者认为这是由于其受体部分存在氰基或酮基而具有 一定的亲水性.

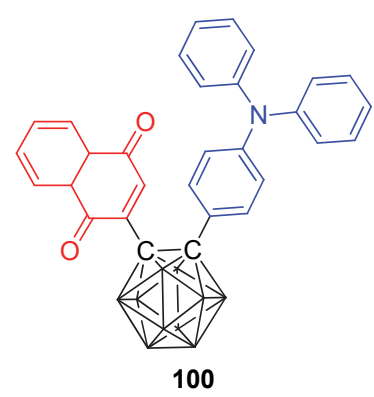

Yasuda 课题组 ${ }^{[79]}$ 以吩噻嗪衍生物为受体设计了系 列 TADF 蓝光材料(分子 $101 \sim 104$ ). 其中, 101 和 103 具 有 AIE 性质. 四个分子的 $\Delta E_{\mathrm{ST}}$ 分别为 $0.08 、 0.06 、 0.11$ 、 $0.11 \mathrm{eV}$, 在掺杂膜中 PLQY 为 $100 \% 、 100 \% 、 92 \% 、 92 \%$. 101 和 103 在纯膜中 PLQY 为 $74 \% 、 83 \%$. 掺杂器件结 构为 ITO $(50 \mathrm{~nm}) / \mathrm{HAT}-\mathrm{CN}(10 \mathrm{~nm}) / \mathrm{TAPC}(50 \mathrm{~nm}) / \mathrm{CCP}$ $(10 \mathrm{~nm}) / \mathrm{EML}(20 \mathrm{~nm}) / \mathrm{PPF}(10 \mathrm{~nm}) / \mathrm{B} 3 \mathrm{PyPB}(30 \mathrm{~nm}) / \mathrm{Liq}$ $(1 \mathrm{~nm}) / \mathrm{Al}(100 \mathrm{~nm})$, 基于 $\mathbf{1 0 1}$ 的器件最大 CE、PE、EQE 分别为 $48.7 \mathrm{~cd} \cdot \mathrm{A}^{-1}, 48.7 \mathrm{~lm} \cdot \mathrm{W}^{-1}$ 和 $27.6 \%$. 基于 101 和 103 的非掺杂器件, 发射蓝光波长都为 $473 \mathrm{~nm}, \mathrm{EQE}$ 分 别为 $20.9 \%$ 和 $17.4 \%$.

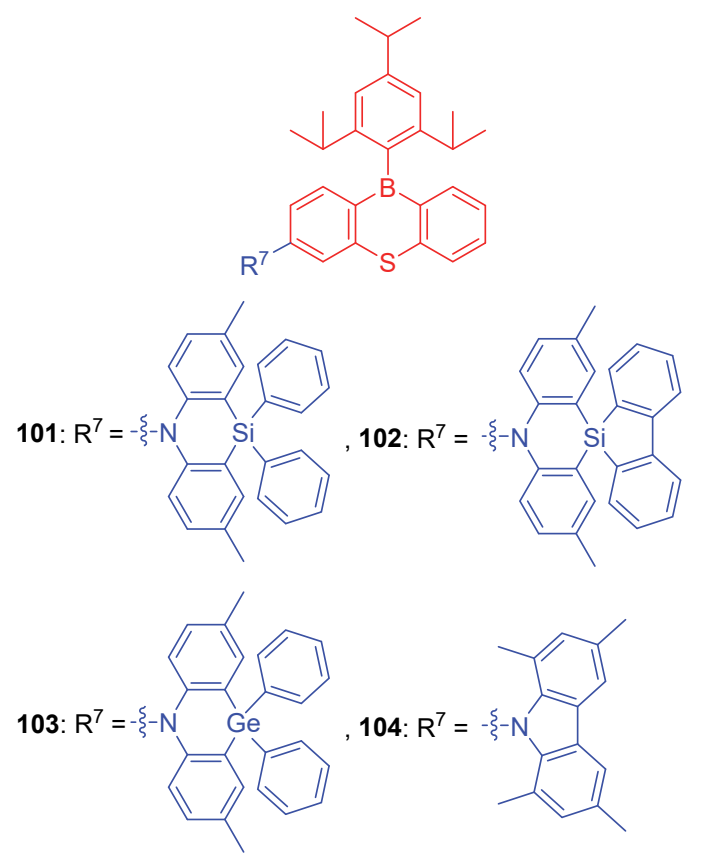

2020 年, 王利祥课题组 ${ }^{[80]}$ 通过在聚苯乙烯链上连 接带有叔丁基、氢、氟取代基的氧桥联三苯基硼受体和 苯基连接的 9,10-二氢鲐啶供体, 控制受体含量合成了 系列通过 TSCT 发光的蓝光聚合物(分子 105 110). 六 个分子表现出 $444 \sim 480 \mathrm{~nm}$ 的蓝光发射, $\Delta E_{\mathrm{ST}}$ 小于 0.10 $\mathrm{eV}$, 延迟苂光在 $0.11 \sim 0.25 \mu \mathrm{s}$ 之间. 用溶液加工的方法 制备结构为 ITO/PEDOT:PSS (40 nm)/TSCT polymer (40 $\mathrm{nm}) / \mathrm{TSPO} 1(8 \mathrm{~nm}) / \mathrm{TmPyPB}(42 \mathrm{~nm}) / \mathrm{LiF}(1 \mathrm{~nm}) / \mathrm{Al}(100$ $\mathrm{nm})$ 的非掺杂器件, 110 获得了最好的 EL 性能, 最大 CE、 $P E 、 E Q E$ 分别为 $30.7 \mathrm{~cd} \cdot \mathrm{A}^{-1}, 30.2 \mathrm{~lm} \bullet \mathrm{W}^{-1}, 15.0 \%$, 其发 
射波长为 $474 \mathrm{~nm}$, 色坐标为 $(0.16,0.27)$, 这是目前性能 最优异的蓝光 TADF-OLED 之一.
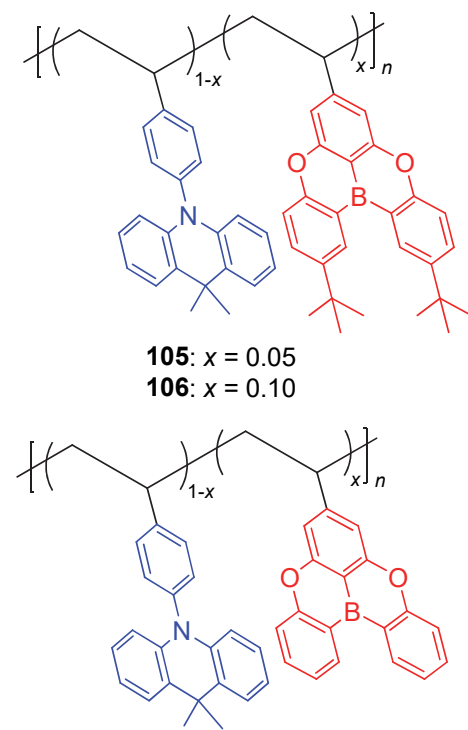

107: $x=0.05$

108: $x=0.10$

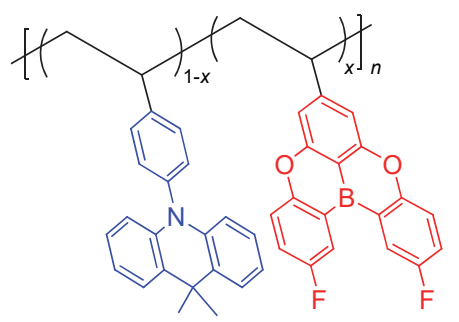

109: $x=0.05$

110: $x=0.10$

孙岳明课题组 ${ }^{[81]}$ 以具有 TADF 性质的 $5 \mathrm{CzBN}$ 为受 体设计了三个分子 111 到 113. 随着给体数量的增加, 受 体部位的 ACQ 被抑制且产生显著的 AIE. 三个分子的 $\Delta E_{\mathrm{ST}}$ 分别为 $0.16 、 0.15 、 0.13 \mathrm{eV}$, 纯膜中的 PLQY 为 $38.7 \% 、 45.7 \%, 69.6 \%$, 延迟寿命为 $3.1 、 4.8 、 3.5 \mu \mathrm{s}$. 溶 液加工制备了结构为 ITO/PEDOT:PSS (40 nm)/EML (40 $\mathrm{nm}) / \mathrm{PO}-\mathrm{T} 2 \mathrm{~T}(40 \mathrm{~nm}) / \mathrm{Cs}_{2} \mathrm{CO}_{3}(2 \mathrm{~nm}) / \mathrm{Al}(100 \mathrm{~nm})$ 的非掺 杂器件, 基于 113 的器件获得了最好的 $\mathrm{EL}$ 性能, 最大 $C E 、 P E 、 E Q E$ 分别为 $58.7 \mathrm{~cd} \cdot \mathrm{A}^{-1}, 46.2 \mathrm{~lm} \cdot \mathrm{W}^{-1}, 20.1 \%$. 这是溶液加工的性能最优异的非掺杂 AIDF-OLED 之 一.

随后, 该课题组 ${ }^{[82]}$ 以 $4 \mathrm{CzIPN}$ 为受体, 通过在给体 上加入甲氧基以及插入苯基等手段设计了两个分子

114、115. 114 仅展现出 TADF 效应, 115 因为在给体受 体之间插入了苯基, 减弱了给体间的空间阻力, 产生了 AIE. 两个分子的 $\Delta E_{\mathrm{ST}}$ 分别为 $0.09 、 0.01 \mathrm{eV}$, 在纯膜中 的 PLQY 为 $35 \%$ 和 $86 \%$, 延迟寿命为 $0.85 、 0.32 \mu \mathrm{s}$. 溶 液加工制备结构为 ITO/PEDOT:PSS $(60 \mathrm{~nm}) / \mathrm{EML}(40$ $\mathrm{nm}) / \mathrm{TPBi}(30 \mathrm{~nm}) / \mathrm{Cs}_{2} \mathrm{CO}_{3}(2 \mathrm{~nm}) / \mathrm{Al}(100 \mathrm{~nm})$ 的非掺杂
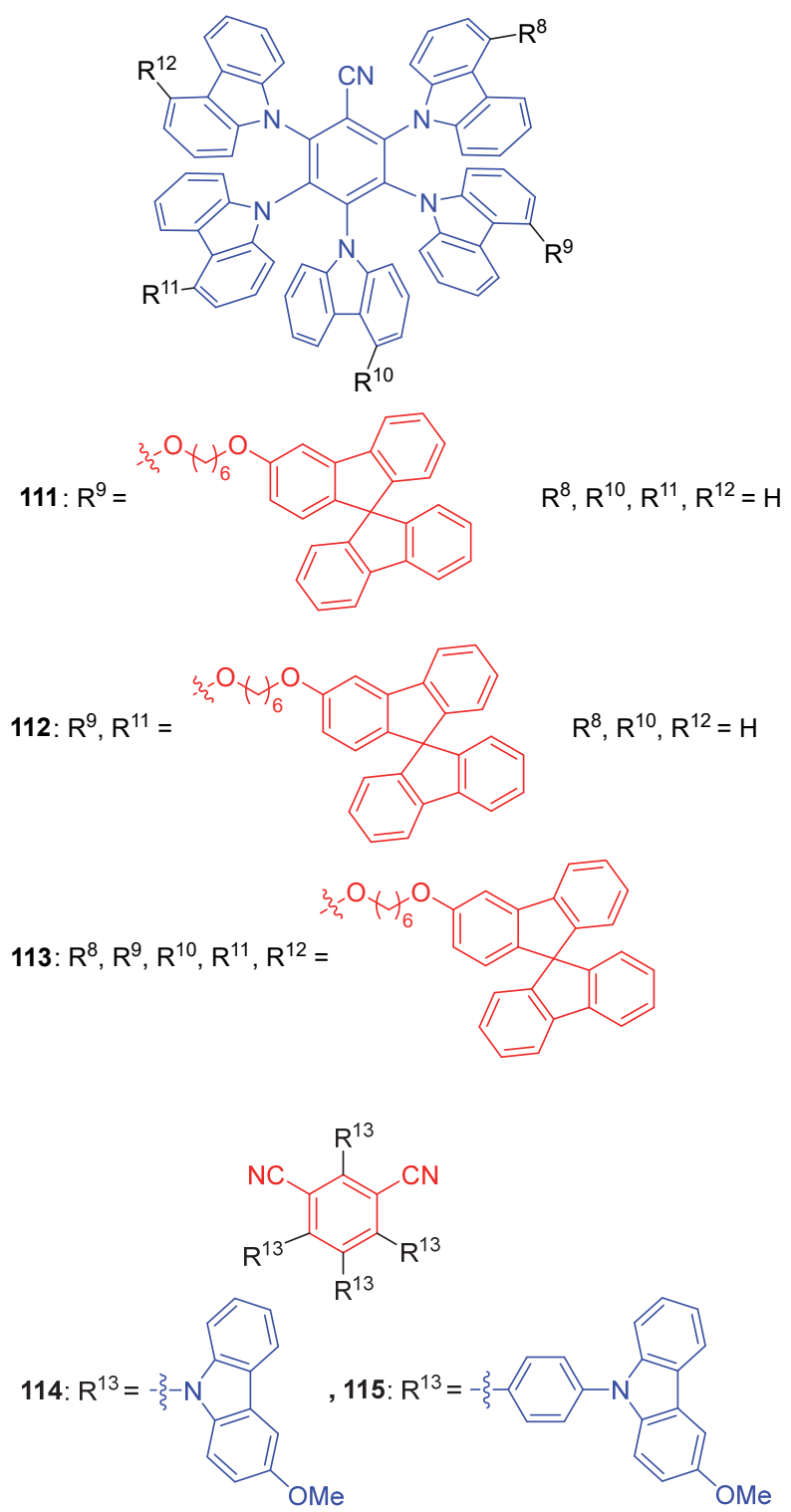

器件, 115 获得更好的 $\mathrm{EL}$ 性能为 $45.1 \mathrm{~cd} \cdot \mathrm{A}^{-1}, 36.0 \mathrm{~lm}$ • $\mathrm{W}^{-1}$ 和 $14.5 \%$, 其在掺杂器件中的最大 CE、PE、 $\mathrm{EQE}$ 分别为 $44.2 \mathrm{~cd} \cdot \mathrm{A}^{-1}, 16.9 \mathrm{~lm} \cdot \mathrm{W}^{-1}$ 和 $16.2 \%$.

\section{6 总结和展望}

综上, AIDF 分子拥有 TADF 的性质, 能同时利用单 线态和三线态发光实现 $100 \%$ 的内量子效率，加以独特 的 AIE 现象，能够有效抑制浓度猝灭和激子猝灭，从而 获得极高的固态 PLQY. 此外, AIDF 材料在聚集态下, 由于分子内运动受限导致内转化通道等非辐射跃迁途 径被限制, 激子可以历经 ISC 和 RISC 通道回到基态发 出显著的延迟苂光; 并且相对于主客体掺杂薄膜中, 在 纯膜中没有主客体能量转移过程, 有利于降低 $\Delta E_{\mathrm{ST}}$ 并 且缩短寿命. 因此, AIDF 分子可以克服掺杂 OLED 中传 统 TADF 发光材料在高亮度下效率大幅降低的瓶颈问 
题, 能够同时兼具优异的固态发光和较高的激子利用 率, 其高度扭曲的分子构型和弱的分子间相互作用使得 高浓度激子的 Dexter 能量转移猝灭效应大大降低, 三重 态激子能够高效快速地转换为单重态激子, 从而降低效 率滚降, 获得优异的电致发光性能. 以此制备的非掺杂 器件制造工艺简单, EL 性能优异, 在高亮度下的效率滚 降极低, 显示出较高的效率稳定性等优点, 从而在 OLED 应用领域上表现出巨大的潜力 ${ }^{[83-84]}$.

基于不同的受体设计, AIDF 分子体系已得到众多 研究, 从最典型的二苯甲酮体系, 到具有特殊四面体结 构的二苯砜, 通过空间进行电荷转移的三嗪, 具有大共 轭平面和刚性结构的喹喔啉, 以及其他各类新型受体 等, 在选择合适给受体进行分子调控的过程中, 始终坚 持以下原则: 使 HOMO、LUMO 充分分离以获得小的 $\Delta E_{\mathrm{ST}}$, 实现高效 RISC; 使 HOMO、LUMO 有一定程度 的重叠, 以保较大电子跃迁几率, 获得高的 PLQY; 使 用巧妙的给受体连接方式以获得扭曲的构象, 保证 AIE 的产生.

自 2017 年 AIDF 被明确提出后, 这一领域发展时间 并不长, 存在某些不足, 例如目前被报道的 AIDF 体系 较少, 可供选择的给受体不多, 且大多是基于已有的 $\mathrm{TADF}$ 分子体系派生而来. 如何开发更具特色, 性能更 优异的分子体系是进一步发展的突破口. 与此同时, 随 着越来越多 AIDF 分子被开发, RIM 机理尽管具有普适 性, 却并不能完全解释其独特现象的产生, 虽然已有诸 多关于阐释特定 AIDF 分子机理的报道, 但关于 AIDF 机理的研究还需要进一步的探索.

由于 D-A 型 AIDF 在设计原则上的固有矛盾: 采用 强给受体单元通过扭曲的分子构象实现有效的前线轨 道分离, 这将不可避免地加重其分子内电荷转移效应, 从而致使发光光谱红移, 难以实现深蓝发射; 此外, 由 于分子结构具有非刚性的外部基团或强振动的官能团 等结构, 并受限于 “能隙定律”, 激发态能量更容易通 过非辐射跃迁形式散失, 导致其难以实现红光或近红外 AIDF 分子发射. 以上原因导致 AIDF 分子在深蓝光、深 红光上难以实现高效电致发光. 这需要设计开发新型的 能级适配的电子供体和受体单元, 以及合理的给受体连 接方式及位点调控.

此外, AIDF 分子因其独特的聚集诱导荧光增强性 质而在非掺杂 OLED 领域有巨大潜力, 不仅简化器件制 备工艺, 同时节约制造成本, 造价更低. 但是, 即便是 上述 EL 性能最优异的非掺杂器件(分子 16, EQE 22.6\%) 和掺杂器件相比依然有一定差距(分子 46, EQE 28.7\%). 提高 AIDF 材料在纯膜状态下的 PLQY 和提高其水平取 向偶极矩从而提升其光输出耦合系数(突破现有的
$20 \%$ ３0\%)可以很好地解决这一问题. 同时，开发高效 的可溶加工热活化延迟苂光材料以摆脱只能通过传统 的真空热蒸镀工艺加工的困境, 亦是降低制作 OLED 器 件工艺成本的重要任务. 结合最近热门的空间限制电荷 转移效应以及大刚性平面的多重共振效应，可预见为设 计更高效 AIDF 分子的良好选择, 并提升 AIDF 分子在 非掺杂器件上的 $\mathrm{EL}$ 性能, 是实现其真正应用于商业化 OLED 上的关键.

\section{References}

[1] Qiu, Z. P.; Tan, J. H.; Cai, N.; Wang, K.; Ji, S. M.; Huo, Y. P. Chin. J. Org. Chem. 2019, 39, 679 (in Chinese). (邱志鹏, 谭继华, 蔡宁, 王凯, 籍少敏, 霍延平, 有机化学, 2019, 39, 679.)

[2] Tonzola, C. J.; Kulkarni, A. P.; Gifford, A. P.; Kaminsky, W.; Jenekhe, S. A. Adv. Funct. Mater. 2007, 17, 863.

[3] Han, C. M.; Xu, H. Chin. Sci. Bull. 2019, 64, 663 (in Chinese). (韩春苗, 许辉, 科学通报, 2019, 64, 663.)

[4] Tang, C. W.; Vanslyke, S. A. Appl. Phys. Lett. 1987, 51, 913.

[5] Baldo, M. A.; O'brien, D. F.; Thompson, M. E.; Forrest, S. R. Phys. Rev. B 1999, 60, 14422.

[6] Turro, N. J.; Ramamurthy, V.; Scaiano, J. C. Photochem. Photobiol. 2012, 88, 1033.

[7] Tan, J. H.; Huo, Y. P.; Cai, N.; Ji, S. M.; Li, Z. Z.; Zhang, L. Chin. J. Org. Chem. 2017, 37, 2480 (in Chinese). (谭继华, 霍延平, 蔡宁, 籍少敏, 李宗植, 张力, 有机化学, 2017, 37, 2480.)

[8] Zhang, Q. S.; Li, B.; Huang, S. P.; Nomura, H.; Tanaka, H.; Adachi, C. Nat. Photonics 2014, 8, 326.

[9] Sato, K.; Shizu, K.; Yoshimura, K.; Kawada, A.; Miyazaki, H.; Adachi, C. Phys. Rev. Lett. 2013, 110, 247401.

[10] Baleizão, C.; Nagl, S.; Borisov, S. M.; Schäferling, M.; Wolfbeis, O. S.; Berberan-Santos, M. N. Chem.-Eur. J. 2007, 13, 3643.

[11] Tao, Y.; Yuan, K.; Chen, T.; Xu, P.; Li, H. H.; Chen, R. F.; Zheng, C.; Zhang, L.; Huang, W. Adv. Mater. 2014, 26, 7931.

[12] Zheng, C. J.; Liu, C. L.; Wang, K.; Tao, S. L.; Lin, H.; Lee, C. S. Sci. China: Chem. 2017, 60, 504

[13] Zhao, Z. F.; Wang, L. D.; Zhan, G.; Liu, Z. W.; Bian, Z. Q.; Huang, C. H. Natl. Sci. Rev. 2021, 8 ,

[14] Guo, J. J.; Zhao, Z. J.; Tang, B. Z. Adv. Opt. Mater. 2018, 6, 1800264.

[15] Adachi, C.; Baldo, M. A.; Thompson, M. E.; Forrest, S. R. J. Appl. Phys. 2001, 90, 5048.

[16] Sasabe, H.; Kido, J. Eur. J. Org. Chem. 2013, 2013, 7653.

[17] Minaev, B.; Baryshnikov, G.; Agren, H. Phys. Chem. Chem. Phys. 2014, 16, 1719.

[18] Zhao, J.; Feng, Z.; Zhong, D. K.; Yang, X. L.; Wu, Y.; Zhou, G. J.; Wu, Z. X. Chem. Mater. 2018, 30, 929.

[19] Zhao, J.; Dang, F. F.; Feng, Z.; Liu, B. A.; Yang, X. L.; Wu, Y.; Zhou, G. J.; Wu, Z. X.; Wong, W. Y. Chem. Commun. 2017, 53, 7581.

[20] Sagara, Y.; Shizu, K.; Tanaka, H.; Miyazaki, H.; Goushi, K.; Kaji, H.; Adachi, C. Chem. Lett. 2015, 44, 360.

[21] Birks, J. B. Science 1971, 174, 580.

[22] Jenekhe, S. A.; Osaheni, J. A. Science 1994, 265, 765.

[23] Zhu, M. R.; Yang, C. L. Chem. Soc. Rev. 2013, 42, 4963.

[24] Im, Y.; Byun, S. Y.; Kim, J. H.; Lee, D. R.; Oh, C. S.; Yook, K. S.; Lee, J. Y. Adv. Funct. Mater. 2017, 27, 1603007.

[25] Cao, X. D.; Zhang, D.; Zhang, S. M.; Tao, Y. T.; Huang, W. J. Mater. Chem. C 2017, 5, 7699.

[26] Luo, J. D.; Xie, Z. L.; Lam, J. W. Y.; Cheng, L.; Chen, H. Y.; Qiu, C. F.; Kwok, H. S.; Zhan, X. W.; Liu, Y. Q.; Zhu, D. B.; Tang, B. Z. Chem. Commun. 2001, 381, 1740. 
[27] Guo, J. J.; Fan, J. Z.; Lin, L. L.; Zeng, J. J.; Liu, H.; Wang, C. K.; Zhao, Z. J.; Tang, B. Z. Adv. Sci. 2019, 6, 1801629.

[28] Xie, Z. Q.; Yang, B.; Cheng, G.; Liu, L. L.; He, F.; Shen, F. Z.; Ma, Y. G.; Liu, S. Y. Chem. Mater. 2005, 17, 1287.

[29] Choi, S.; Bouffard, J.; Kim, Y. Chem. Sci. 2014, 5, 751

[30] Sonoda, Y.; Tsuzuki, S.; Goto, M.; Tohnai, N.; Yoshida, M. J. Phys. Chem. A 2010, 114, 172

[31] Mutai, T.; Sawatani, H.; Shida, T.; Shono, H.; Araki, K. J. Org Chem. 2013, 78, 2482.

[32] Ding, D.; Li, K.; Liu, B.; Tang, B. Z. Acc. Chem. Res. 2013, 46, 2441.

[33] Hu, R. R.; Lager, E.; Aguilar-Aguilar, A.; Liu, J. Z.; Lam, J. W. Y.; Sung, H. H. Y.; Williams, I. D.; Zhong, Y. C.; Wong, K. S.; Pena-Cabrera, E.; Tang, B. Z. J. Phys. Chem. C 2009, 113, 15845.

[34] Kondakov, D. Y. J. Soc. Inf. Disp. 2009, 17, 137.

[35] Yang, C. L. Sci. China: Chem. 2021, 64, 165 (in Chinese). (杨楚罗, 中国科学: 化学, 2021, 64, 165).

[36] Guo, J. J.; Li, X. L.; Nie, H.; Luo, W. W.; Gan, S. F.; Hu, S. M.; Hu, R. R.; Qin, A. J.; Zhao, Z. J.; Su, S. J.; Tang, B. Adv. Funct. Mater. 2017, 27, 1606458.

[37] Huang, J.; Nie, H.; Zeng, J. J.; Zhuang, Z. Y.; Gan, S. F.; Cai, Y. J.; Guo, J. J.; Su, S. J.; Zhao, Z. J.; Tang, B. Z. Angew. Chem., Int. Ed. Engl. 2017, 56, 12971 .

[38] Chen, Y. H.; Wang, S. M.; Wu, X. F.; Xu, Y. X.; Li, H.; Liu, Y.; Tong, H.; Wang, L. X. J. Mater. Chem. C 2018, 6, 12503.

[39] Zhao, Y. D.; Wang, W. G.; Gui, C.; Fang, L.; Zhang, X. L.; Wang, S. J.; Chen, S. M.; Shi, H. P.; Tang, B. Z. J. Mater. Chem. C 2018, 6, 2873.

[40] Ma, F. L.; Cheng, Y.; Zheng, Y.; Ji, H. F.; Hasrat, K.; Qi, Z. J. J. Mater. Chem. C 2019, 7, 9413

[41] Zheng, K. L.; Yang, H. N.; Ni, F.; Chen, Z. X.; Gong, S. L.; Lu, Z. H.; Yang, C. L. Adv. Opt. Mater. 2019, 7, 1900727.

[42] Zeng, J. J.; Guo, J. J.; Liu, H.; Zhao, Z. J.; Tang, B. Z. Adv. Funct. Mater. 2020, 30, 2000019.

[43] Lee, J.; Aizawa, N.; Numata, M.; Adachi, C.; Yasuda, T. Adv. Mater. 2017, 29, 1604856

[44] Zhang, D. D.; Wei, P. C.; Zhang, D. Q.; Duan, L. ACS Appl. Mater. Interfaces 2017, 9, 19040.

[45] Liu, H. J.; Zeng, J. J.; Guo, J. J.; Nie, H.; Zhao, Z. J.; Tang, B. Z. Angew. Chem., Int. Ed.. 2018, 57, 9290.

[46] Fu, Y.; Liu, H. J.; Zhu, X. Y.; Zeng, J. J.; Zhao, Z. J.; Tang, B. Z. J. Mater. Chem. C 2020, 8, 9549.

[47] Tan, J. H.; Chen, W. C.; Ni, S. F.; Qiu, Z. P.; Zhan, Y. Y.; Yang, Z. W.; Xiong, J. W.; Cao, C.; Huo, Y. P.; Lee, C. S. J. Mater. Chem. C 2020, 8, 8061 .

[48] Yang, Z. W.; Zhan, Y. Y.; Qiu, Z. P.; Zeng, J. J.; Guo, J. J.; Hu, S.; Zhao, Z. J.; Li, X. W.; Ji, S. M.; Huo, Y. P.; Su, S. J. ACS Appl. Mater. Interfaces 2020, 12, 29528.

[49] Qiu, Z. P.; Xie, W. T.; Yang, Z. W.; Tan, J. H.; Yuan, Z. X.; Xing, L. J.; Ji, S. M.; Chen, W. C.; Huo, Y. P.; Su, S. J. Chem. Eng. J. 2021, $415,128949$.

[50] Huang, J.; Xu, Z.; Cai, Z. Y.; Guo, J. J.; Guo, J. L.; Shen, P. C.; Wang, Z. M.; Zhao, Z. J.; Ma, D. G.; Tang, B. Z. J. Mater. Chem. C 2019, 7, 330 .

[51] Liu, Y.; Wu, X. F.; Chen, Y. H.; Chen, L.; Li, H.; Wang, W. J.; Wang, S. M.; Tian, H. K.; Tong, H.; Wang, L. X. J. Mater. Chem. C 2019, 7, 9719 .

[52] Ma, F. L.; Zhao, X. X.; Ji, H. F.; Zhang, D. D.; Hasrat, K.; Qi, Z. J. J. Mater. Chem. C 2020, 8, 12272.

[53] Dorman, G.; Prestwich, G. D. Biochemistry 1994, 33, 5661

[54] Li, C. S.; Nobuyasu, R. S.; Wang, Y. K.; Dias, F. B.; Ren, Z. J.; Bryce, M. R.; Yan, S. K. Adv. Opt. Mater. 2017, 5, 1700435.

[55] Wei, X. F.; Chen, Y. Z.; Duan, R. H.; Liu, J. J.; Wang, R. F.; Liu, Y. W.; Li, Z. Y.; Yi, Y. P.; Yamada-Takamura, Y.; Wang, P. F.; Wang, Y. J. Mater. Chem. C 2017, 5, 12077.

[56] Xiang, S. P.; Huang, Z.; Sun, S. Q.; Lv, X. L.; Fan, L. W.; Ye, S. F.;
Chen, H. T.; Guo, R. D.; Wang, L. J. Mater. Chem. C 2018, 6, 11436

[57] Yang, Z.; Mao, Z.; Xu, C.; Chen, X. J.; Zhao, J.; Yang, Z. Y.; Zhang, Y.; Wu, W.; Jiao, S. B.; Liu, Y.; Aldred, M. P.; Chi, Z. G. Chem. Sci. 2019, 10, 8129.

[58] Zhan, L. S.; Xiang, Y. P.; Chen, Z. X.; Wu, K. L.; Gong, S. L.; Xie, G. H.; Yang, C. L. J. Mater. Chem. C 2019, 7, 13953.

[59] Tsujimoto, H.; Ha, D. G.; Markopoulos, G.; Chae, H. S.; Baldo, M. A.; Swager, T. M. J. Am. Chem. Soc. 2017, 139, 4894.

[60] Wang, X. D.; Wang, S. M.; Lv, J. H.; Shao, S. Y.; Wang, L. X.; Jing, X. B.; Wang, F. S. Chem. Sci. 2019, 10, 2915.

[61] Park, S. Y.; Choi, S.; Park, G. E.; Kim, H. J.; Lee, C.; Moon, J. S.; Kim, S. W.; Park, S.; Kwon, J. H.; Cho, M. J.; Choi, D. H. ACS Appl. Mater. Interfaces 2018, 10, 14966.

[62] Zhang, Q.; Sun, S. Q.; Liu, W.; Leng, P. P.; Lv, X. L.; Wang, Y. X.; Chen, H. T.; Ye, S. F.; Zhuang, S. Q.; Wang, L. J. Mater. Chem. C 2019, 7, 9487

[63] Wang, Y. F.; Lu, H. Y.; Shen, Y. F.; Li, M.; Chen, C. F. Chem. Commun. 2019, 55, 9559.

[64] Pope, M.; Kallmann, H. P.; Magnante, P. J. J. Chem. Phys. 2004, $38,2042$.

[65] Kim, M.; Jeon, S. K.; Hwang, S. H.; Lee, S. S.; Yu, E.; Lee, J. Y. Chem. Commun. 2016, 52, 339.

[66] Yu, L.; Wu, Z. B.; Xie, G. H.; Zeng, W. X.; Ma, D. G.; Yang, C. L. Chem. Sci. 2018, 9, 1385.

[67] Yu, L.; Wu, Z. B.; Xie, G. H.; Zhong, C.; Zhu, Z. C.; Ma, D. G.; Yang, C. L. Chem. Commun. 2018, 54, 1379.

[68] Pashazadeh, R.; Sych, G.; Nasiri, S.; Leitonas, K.; Lazauskas, A. Volyniuk, D.; Skabara, P. J.; Grazulevicius, J. V. Chem. Eng. J. 2020, 401, 125962

[69] Liu, Y.; Chen, Y. H.; Li, H.; Wang, S.; Wu, X. F.; Tong, H.; Wang, L. X. ACS Appl. Mater. Interfaces 2020, 12, 30652.

[70] Zheng, K. L.; Ni, F.; Chen, Z. X.; Zhong, C.; Yang, C. L. Angew. Chem., Int. Ed. Engl. 2020, 59, 9972.

[71] Yang, W.; Yang, Y. Y.; Zhan, L. S.; Zheng, K. L.; Chen, Z. X.; Zeng, X.; Gong, S. L.; Yang, C. L. Chem. Eng. J. 2020, 390, 124626

[72] Huang, B.; Li, Z. J.; Yang, H.; Hu, D.; Wu, W. J.; Feng, Y.; Sun, Y. M.; Lin, B. P.; Jiang, W. J. Mater. Chem. C 2017, 5, 12031.

[73] Hladka, I.; Volyniuk, D.; Bezvikonnyi, O.; Kinzhybalo, V.; Bednarchuk, T. J.; Danyliv, Y.; Lytvyn, R.; Lazauskas, A.; Grazulevicius, J. V. J. Mater. Chem. C 2018, 6, 13179.

[74] Song, F. Y.; Xu, Z.; Zhang, Q. S.; Zhao, Z.; Zhang, H. K.; Zhao, W. J.; Qiu, Z. J.; Qi, C. X.; Zhang, H.; Sung, H. H. Y.; Williams, I. D.; Lam, J. W. Y.; Zhao, Z. J.; Qin, A. J.; Ma, D. G.; Tang, B. Z. Adv. Funct. Mater. 2018, 28, 1800051.

[75] Chen, C.; Lu, H. Y.; Wang, Y. F.; Li, M.; Shen, Y. F.; Chen, C. F. J. Mater. Chem. C 2019, 7, 4673.

[76] Zhou, X.; Yang, H. N.; Chen, Z. X.; Gong, S. L.; Lu, Z. H.; Yang, C. L. J. Mater. Chem. C 2019, 7, 6607.

[77] Chen, S.; Zeng, P. J.; Wang, W. G.; Wang, X. D.; Wu, Y. K.; Lin, P. J.; Peng, Z. C. J. Mater. Chem. C 2019, 7, 2886.

[78] Duan, Y. C.; Gao, Y.; Geng, Y.; Wu, Y.; Shan, G. G.; Zhao, L.; Zhang, M.; Su, Z. M. J. Mater. Chem. C 2019, 7, 2699.

[79] Matsuo, K.; Yasuda, T. Chem. Sci. 2019, 10, 10687.

[80] Chen, F.; Hu, J.; Wang, X. D.; Shao, S. Y.; Wang, L. X.; Jing, X. B.; Wang, F. S. Sci. China: Chem. 2020, 63, 1112.

[81] Liu, D.; Wei, J. Y.; Tian, W. W.; Jiang, W.; Sun, Y. M.; Zhao, Z.; Tang, B. Z. Chem. Sci. 2020, 11, 7194.

[82] Sun, K. Y.; Liu, D.; Tian, W. W.; Gu, F.; Wang, W. X.; Cai, Z. S.; Jiang, W.; Sun, Y. M. J. Mater. Chem. C 2020, $8,11850$.

[83] Zhang, Q. S.; Zhou, Q. G.; Cheng, Y. X.; Wang, L. X.; Ma, D. G.; Jing, X. B.; Wang, F. S. Adv. Mater. 2004, 16, 432.

[84] Uoyama, H.; Goushi, K.; Shizu, K.; Nomura, H.; Adachi, C. Nature 2012, 492, 234. 